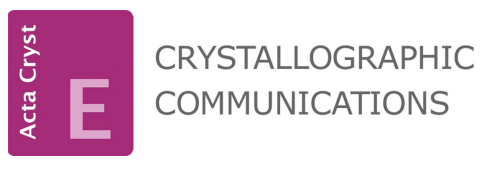

ISSN 2056-9890

Received 16 November 202

Accepted 9 December 2021

Edited by C. Schulzke, Universität Greifswald, Germany

Keywords: crystal structure; 2,6-dimethylphenyl isocyanide; xylylisocyanide; CNXyl; iron; infrared spectroscopy.

CCDC references: $2127598 ; 2127597$; 2127596

Supporting information: this article has supporting information at journals.iucr.org/e

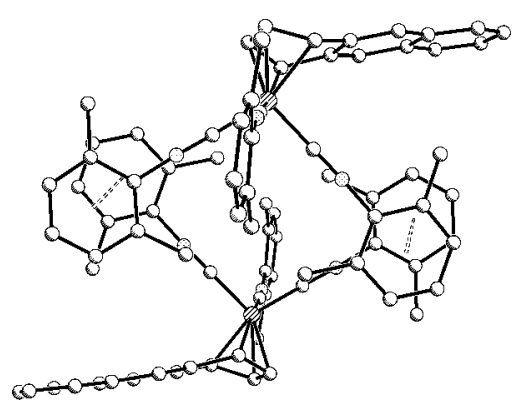

\section{Crystal structures of two novel iron isocyanides from the reaction of 2,6-dimethylphenyl isocyanide, $\mathrm{CNXyl}$, with bis(anthracene)ferrate(-1)}

\author{
William W. Brennessel ${ }^{\mathrm{a} *}$ and John E. Ellis ${ }^{\mathrm{b}}$
}

a Department of Chemistry, 120 Trustee Road, University of Rochester, Rochester, NY 14627, USA, and ${ }^{\mathbf{b}}$ Department of
Chemistry, 207 Pleasant Street SE, University of Minnesota, Minneapolis, MN 55455, USA. *Correspondence e-mail:
william.brennessel@rochester.edu

The reaction of the $\left[\mathrm{K}(18 \text {-crown- } 6)(\text { thf })_{2}\right]^{1+}$ (thf is tetrahydrofuran) salt of bis(anthracene)ferrate $(-1)$, or $\left[\mathrm{Fe}\left(\mathrm{C}_{14} \mathrm{H}_{10}\right)_{2}\right]^{-}$, with 2,6-dimethylphenyl isocyanide $(\mathrm{CNXyl})$ in thf resulted in the formation of two new iron isocyanide complexes, namely, [(1,2,3,4- $\eta$ )-anthracene]tris(2,6-dimethylphenyl isocyanide)iron, $\left[\mathrm{Fe}\left(\mathrm{C}_{14} \mathrm{H}_{10}\right)\left(\mathrm{C}_{9} \mathrm{H}_{9} \mathrm{~N}\right)_{3}\right]$ or $\left[\mathrm{Fe}\left(1,2,3,4-\eta-\mathrm{C}_{14} \mathrm{H}_{10}\right)(\mathrm{CNXyl})_{3}\right]$, and $\{5,6$-bis $(2,6-$ dimethylanilino)-3-(2,6-dimethylphenyl)-1,2,7-tris[(2,6-dimethylphenyl)imino]3-azoniahept-3-ene-1,4,7-triido) tris(2,6-dimethylphenyl isocyanide)iron tetrahydrofuran disolvate, $\left[\mathrm{Fe}\left(\mathrm{C}_{54} \mathrm{H}_{56} \mathrm{~N}_{6}\right)\left(\mathrm{C}_{9} \mathrm{H}_{9} \mathrm{~N}\right)_{3}\right] \cdot 2 \mathrm{C}_{4} \mathrm{H}_{8} \mathrm{O}$ or $\left[\mathrm{Fe}\left(\mathrm{C}_{54} \mathrm{H}_{56} \mathrm{~N}_{6}\right)\right.$ $\left.(\mathrm{CNXyl})_{3}\right] \cdot 2 \mathrm{C}_{4} \mathrm{H}_{8} \mathrm{O}$, which were characterized by single-crystal X-ray diffraction. The former is likely an intermediate along the path to the known homoleptic $\left[\mathrm{Fe}(\mathrm{CNXyl})_{5}\right]$, while the latter contains a tridentate ligand that is formed from the 'coupling' of six CNXyl ligands. A third crystal structure from this reaction, (7-methylindol-1-ido- $\kappa N)(1,4,7,10,13,16$-hexaoxacyclooctadecane$\left.\kappa^{6} \mathrm{O}\right)$ potassium, $\left[\mathrm{K}\left(\mathrm{C}_{9} \mathrm{H}_{8} \mathrm{~N}\right)\left(\mathrm{C}_{12} \mathrm{H}_{24} \mathrm{O}_{6}\right)\right]$ or $\left[\mathrm{K}\left(\mathrm{C}_{9} \mathrm{H}_{8} \mathrm{~N}\right)(18\right.$-crown-6)], contains a 7-methylindol-1-ide anion, in which one $\mathrm{CNXyl}$ ligand has shed a proton during its reductive cyclization.

\section{Chemical context}

The low-valent bis(anthracene)cobaltate $(-1)$ has been shown to be an excellent source of spin-paired atomic $\mathrm{Co}(-1)$ anions in substitution reactions in which both anthracene $\left(\mathrm{C}_{14} \mathrm{H}_{10}\right)$ ligands are readily displaced by a wide variety of acceptor ligands (Brennessel et al., 2002; Brennessel \& Ellis, 2012). The reaction with four equivalents of $\mathrm{CNXyl}$, $\mathrm{Xyl}$ is 2,6-dimethylphenyl, resulted in an excellent yield of the homoleptic isocyanidecobaltate $(-1),\left[\mathrm{Co}(\mathrm{CNXyl})_{4}\right]^{1-}$, first obtained by an alternate synthesis (Warnock \& Cooper, 1989). Attempts to prepare the analogous 18-electron iron complex, bis(anthracene)ferrate( -2$)$, afforded only the related 17-electron, paramagnetic bis(anthracene)ferrate(-1) (Brennessel et al., 2007). The latter species was shown to react with carbon monoxide to afford excellent yields of the $\mathrm{Fe}(-1)$ complex, $\left[\mathrm{Fe}_{2}(\mathrm{CO})_{8}\right]^{2-}$. On this basis, the corresponding reaction with $\mathrm{CNXyl}$ in tetrahydrofuran, thf, was examined to determine whether the unknown $\left[\mathrm{Fe}_{2}(\mathrm{CNXyl})_{8}\right]^{2-}$ could be accessed. Bis(anthracene)ferrate $(-1)$ was also reacted with excess $\mathrm{CNXyl}$ in the presence of one equivalent of a reducing agent to see whether the previously reported monometallic $\left[\mathrm{Fe}(\mathrm{CNXyl})_{4}\right]^{2-}$ (Brennessel \& Ellis, 2007) could be prepared by this facile route. However, in both cases, infrared (IR) spectroscopy indicated predominant formation of the long- 
known, but only recently structurally authenticated $\mathrm{Fe}^{0}$ complex, [ $\left.\mathrm{Fe}(\mathrm{CNXyl})_{5}\right]$ (Bassett et al., 1979, Brennessel et al., 2019).
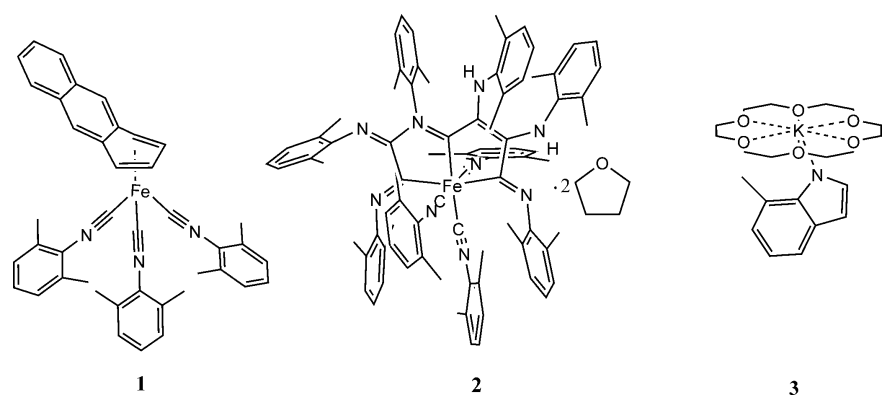

Because a complex containing formally $\mathrm{Fe}^{-\mathrm{I}}$ resulted in an oxidation to $\mathrm{Fe}^{0}$, it was of interest to determine what other species were produced by the reaction of bis(anthracene)ferrate $(-1)$ with excess CNXyl in THF. First an aliquot was taken from the reaction mixture early on and placed in a $243 \mathrm{~K}$ freezer until orange blocks were observed. A single crystal $\mathrm{X}$-ray diffraction experiment revealed these to be $\left[\mathrm{Fe}\left(\mathrm{C}_{14} \mathrm{H}_{10}\right)(\mathrm{CNXyl})_{3}\right] \mathbf{1}$ (Fig. 1). It is thought that this complex is likely a crystallization-trapped intermediate, since $\left[\mathrm{Fe}(\mathrm{CNXyl})_{5}\right]$ is ultimately produced. Compound $\mathbf{1}$ is of interest as the first mixed anthracene-isocyanide derivative of the unknown bis(anthracene)iron(0). However, the related carbonyl, $\left[\mathrm{Fe}\left(\mathrm{C}_{14} \mathrm{H}_{10}\right)(\mathrm{CO})_{3}\right]$, has been known for more than 50 years (Manuel, 1964).

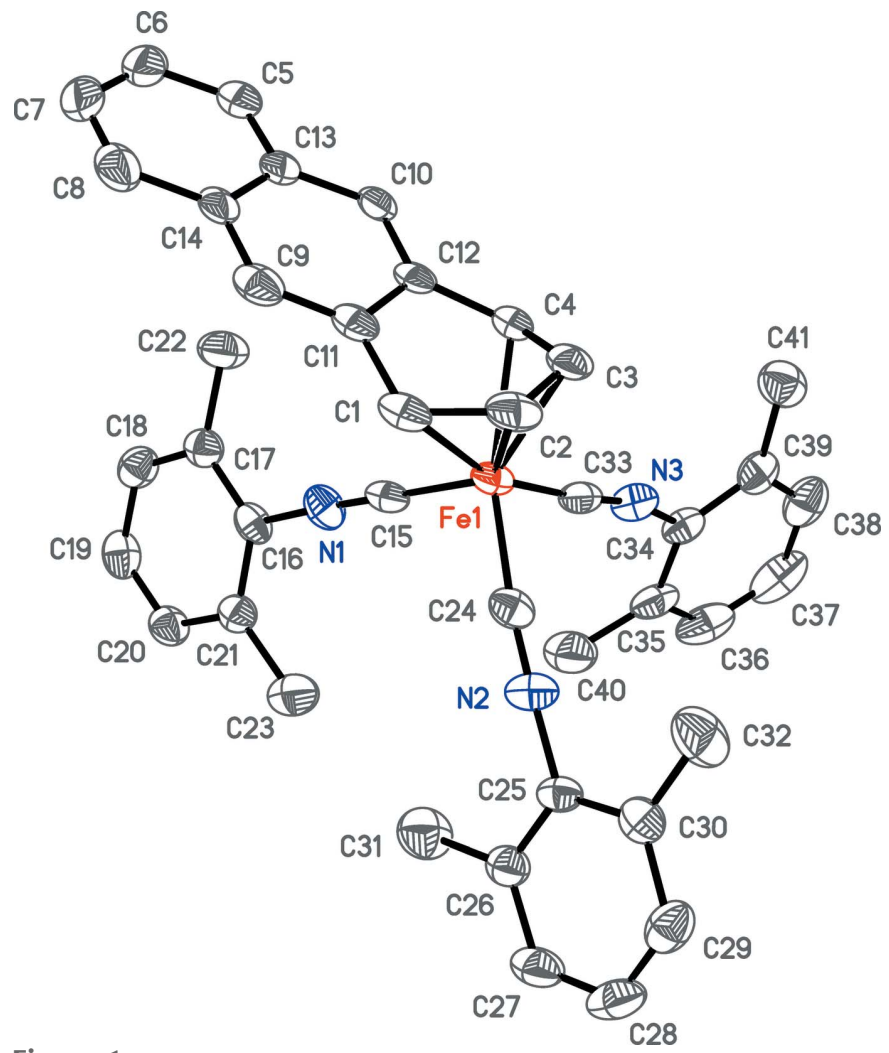

Figure 1

Anisotropic displacement ellipsoid plot of $\mathbf{1}$ drawn at the $50 \%$ probability level with $\mathrm{H}$ atoms omitted.
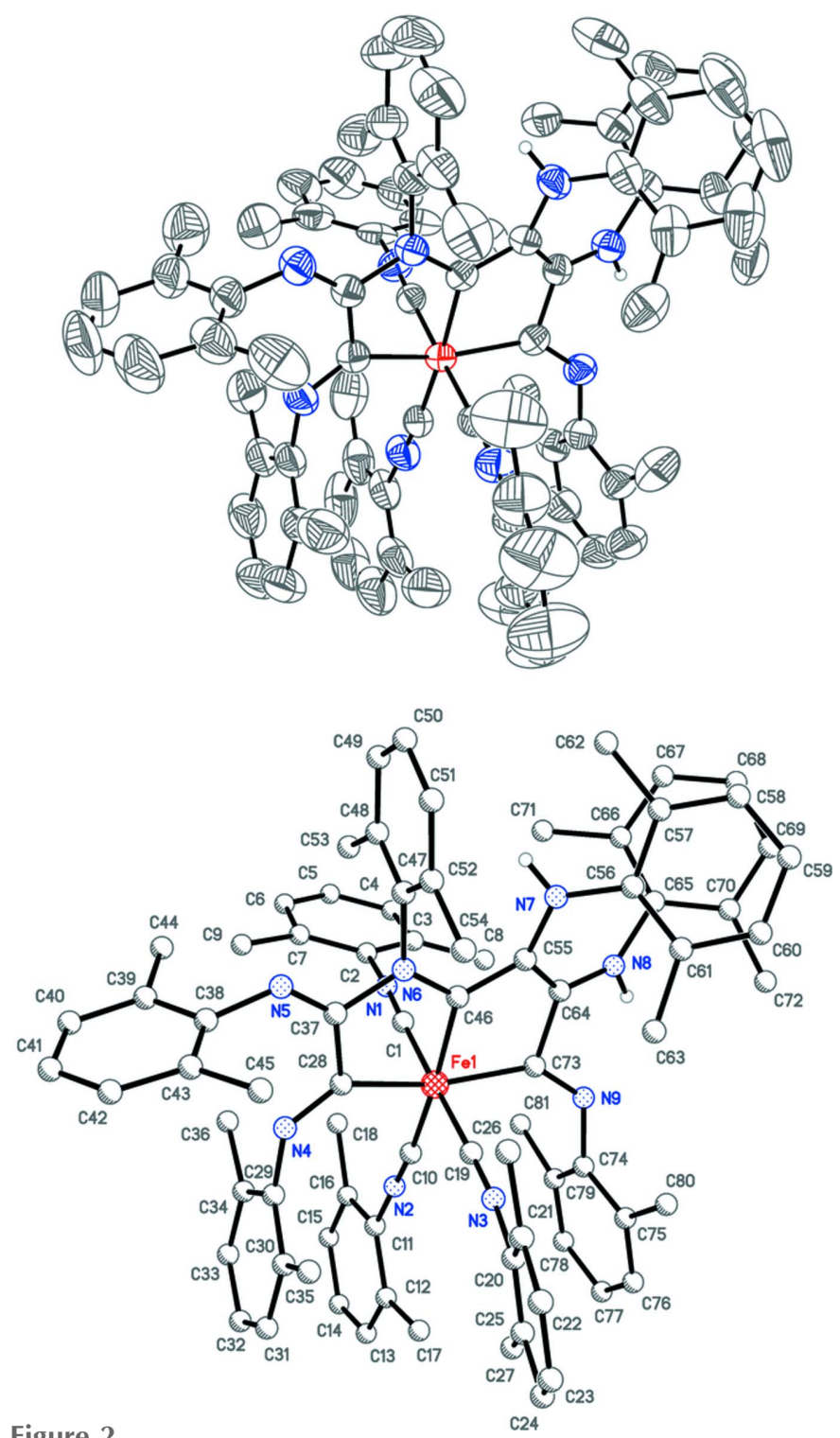

Figure 2

Plots of $\mathbf{2}$ with $\mathrm{C}-\mathrm{H}$ hydrogen atoms and solvent molecules omitted and with only the major components of disorder shown. Top: anisotropic displacement ellipsoid plot drawn at the $50 \%$ probability level. Bottom: ball-and-stick plot in the same orientation featuring the numbering scheme.

After the reaction mixture had warmed to room temperature and stirred for a few hours, the solvent was removed and $n$-heptane was added. The mixture was then filtered and a new species crystallized in the filtrate. A crystal structure revealed the material to be a thf disolvate of $\left[\mathrm{Fe}\left(\mathrm{C}_{54} \mathrm{H}_{56} \mathrm{~N}_{9}\right)(\mathrm{CNXyl})_{3}\right] 2$ (Fig. 2). In this case, six isocyanides had reductively 'coupled' to form a previously unknown tridentate ligand that had been protonated twice at two of the nitrogen atoms (Fig. 3). An IR spectrum obtained from the few crystals that could be harvested showed $\nu \mathrm{CN}$ stretches of $2110 \mathrm{w}$ and $2055 \mathrm{vs} \mathrm{cm}^{-1}$, consistent with an $\mathrm{Fe}^{+2}$ oxidation state, which would make the ligand formally dianionic. The source of the protons in aprotic media was still a mystery at this point.

Coupling of isocyanide ligands has precedent (Yamamoto \& Yamazaki, 1972, Lam et al., 1977, Giandomenico et al., 1982, 


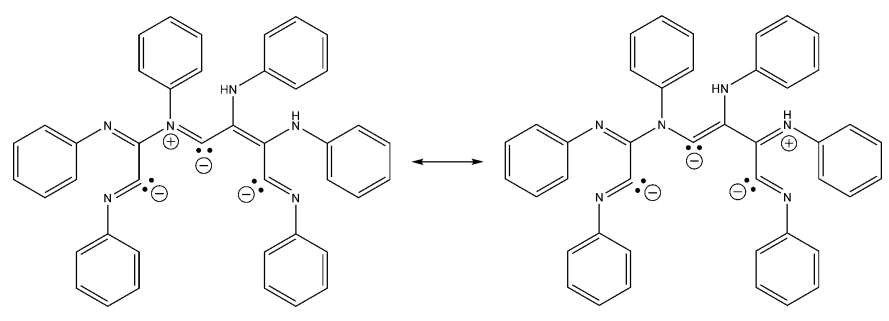

Figure 3

Two proposed resonance forms of the tridentate dianion of $\mathbf{2}$ based on the bond lengths.

Warner \& Lippard, 1986), although this exact 'coupling' of six isocyanide ligands appears to be new. The protonation of nitrogen atoms has also been observed in such circumstances. For instance, reduction of $\left[\mathrm{Mo}(\mathrm{CN} R)_{6} X\right]^{+}$(many variations on $R$ and $X)$ by $\mathrm{Zn}$ in the presence of water generated a bis(alkylamino)acetylene ligand with protonated nitrogen atoms (Lam et al., 1977; Giandomenico et al., 1982; Warner \& Lippard, 1986). The source of protons in the production of $\mathbf{2}$, however, was not discovered until single crystals grown from the heptane-insoluble component were evaluated. The structure was formulated by X-ray diffraction as $[\mathrm{K}(18$-crown6) $\left.\left(\mathrm{C}_{9} \mathrm{H}_{8} \mathrm{~N}\right)\right] 3$ (Fig. 4), a cyclized, reduced form of $\mathrm{CNXyl}$, from which one hydrogen atom was lost. It must be emphasized that examples of trimerization (Yamamoto et al., 1982; Blake et al., 1997; Bashall et al., 2000; Chen et al., 2019), tetramerization (Shen et al., 2014; Altenburger et al., 2016; Kucera et al., 2019), pentamerization (Tanase et al., 1992, 1996), hexamerization (Shen et al., 2014), and polymerization (Yamamoto \& Yama-

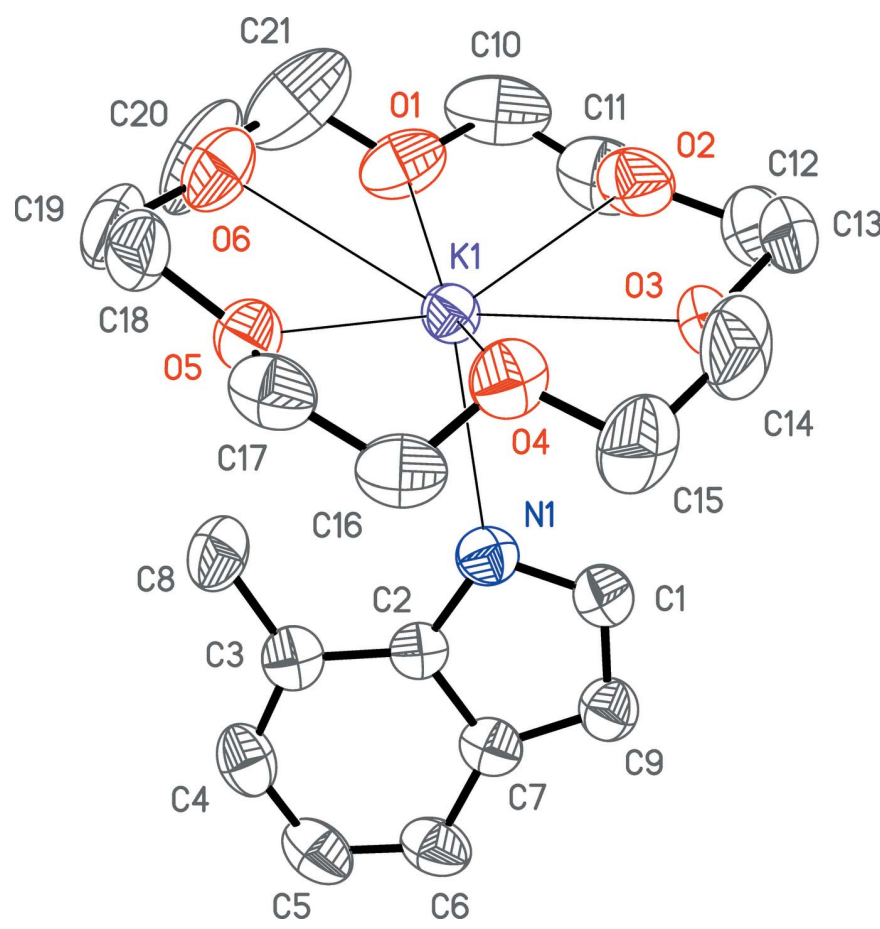

Figure 4

Anisotropic displacement ellipsoid plot of $\mathbf{3}$ drawn at the $50 \%$ probability level with $\mathrm{H}$ atoms and the minor component of disorder omitted. zaki, 1972) of isocyanides are well-precedented, but 2 appears to be only the second example in which hexamerization of an organic isocyanide has been established.

Given the speciation observed by the crystal structures and IR spectroscopy, a balanced equation can be written [Equation (1)]. The hydrogen atom lost during the reduction and cyclization that forms $\mathbf{3}$ is now found in the two protonations in the one-half equivalent of $\mathbf{2}$.

Interestingly, in support of this equation, when less than eight equivalents of CNXyl were employed (e.g., four), intractable tars resulted. It should be noted, however, that this equation is only speculative and requires further investigation for confirmation.

Equation (1)

$\left[\mathrm{K}(18 \text {-crown-6)(thf })_{2}\right]\left[\mathrm{Fe}\left(\mathrm{C}_{14} \mathrm{H}_{10}\right)_{2}\right]+8 \mathrm{CNXyl} \rightarrow$ $0.5\left[\mathrm{Fe}(\mathrm{CNXyl})_{5}\right]+0.5\left[\mathrm{Fe}\left(\mathrm{C}_{54} \mathrm{H}_{56} \mathrm{~N}_{6}\right)(\mathrm{CNXyl})_{3}\right]+[\mathrm{K}(18-$ crown-6) $\left.\left(\mathrm{C}_{9} \mathrm{H}_{8} \mathrm{~N}\right)\right]$

\section{Structural commentary}

The geometry at the formally zerovalent iron center of $\mathbf{1}$ is nearly identical to those of related molecules with one 1,2,3,4$\eta$-naphthalene $o$-anthracene ligand and three excellent acceptor ligands in a tripodal arrangement. The average of the three $(\mathrm{XylN}) \mathrm{C}-\mathrm{Fe}-\mathrm{C}(\mathrm{NXyl})$ angles of $\mathbf{1}, 95.3^{\circ}$, matches well with that of the average $\mathrm{C}-\mathrm{Fe}-\mathrm{C}$ angle from three carbonyl ligands of the $\left[\mathrm{Fe}(1,2,3,4-\eta\right.$-naphthalene $\left.)(\mathrm{CO})_{3}\right]$ portion of a trinuclear molecule, 97.5 (Imhof, 1999), and those of the average $\mathrm{P}-\mathrm{Fe}-\mathrm{P}$ angles from $[\mathrm{Fe}(1,2,3,4-\eta$-naphthalene)$\left.\left(\mathrm{P}(\mathrm{OMe})_{3}\right)_{3}\right], 97.7^{\circ}$ (Schäufele et al., 1989), and [Fe(1,2,3,4- $\eta$ anthracene $\left.)\left(\mathrm{P}(\mathrm{OMe})_{3}\right)_{3}\right], 97.9^{\circ}$ (Brennessel et al., 2007). The 'fold angle' between the iron-coordinating $\eta^{4}$-diene unit and the exo-benzene or -naphthalene portions are 30.7, 30.2, 40.6, and $40.8^{\circ}$, respectively, for the same four structures. The latter two angles are significantly larger than those in molecules containing three $\mathrm{CNXyl}$ or $\mathrm{CO}$ ligands, and since the $\mathrm{Fe}-$ $\mathrm{C}\left(\eta^{4}\right.$-diene) bond lengths (Table 1$)$ in all four structures are comparable, it would be interesting to know if this is an electronic effect due to the different nature of $\mathrm{CO} / \mathrm{CNXyl}$ versus phosphite and/or due to the bulk of the trimethylphosphite ligands.

The ligand set of $\mathbf{2}$ is built from nine CNXyl ligands, of which six, with the addition of two protonations at nitrogen atoms, have joined together into one tridentate dianionic ligand. Because this ligand is essentially planar at the core of two fused metallacyclopentanes (Fig. 2), it binds the iron center meridionally. The three remaining CNXyl ligands are also meridional, resulting in a distorted octahedral geometry. The bond lengths in the fused ring core (Table 2) suggest resonance stabilization (Fig. 3). To our knowledge, only one other 'coupling' of six isocyanide ligands has been structurally verified. In this case, six cyclohexyl isocyanide ligands have 'coupled' into a dianionic ligand (without any protonations) that bridges two chromium centers (Shen et al., 2014).

In $\mathbf{3}$, one $\mathrm{CNXyl}$ molecule has reductively cyclized into a 7-methylindol-1-ide anion (Fig. 4). The potassium cation is 
Table 1

Selected geometric parameters $\left(\AA,{ }^{\circ}\right)$ for $\mathbf{1}$.

\begin{tabular}{lrll}
\hline $\mathrm{Fe} 1-\mathrm{C} 33$ & $1.800(4)$ & $\mathrm{C} 1-\mathrm{C} 2$ & $1.415(5)$ \\
$\mathrm{Fe} 1-\mathrm{C} 24$ & $1.840(3)$ & $\mathrm{C} 2-\mathrm{C} 3$ & $1.399(5)$ \\
$\mathrm{Fe} 1-\mathrm{C} 15$ & $1.847(3)$ & $\mathrm{C} 3-\mathrm{C} 4$ & $1.421(5)$ \\
$\mathrm{Fe} 1-\mathrm{C} 3$ & $2.034(3)$ & $\mathrm{C} 15-\mathrm{N} 1$ & $1.167(4)$ \\
$\mathrm{Fe} 1-\mathrm{C} 2$ & $2.044(3)$ & $\mathrm{C} 24-\mathrm{N} 2$ & $1.165(4)$ \\
$\mathrm{Fe} 1-\mathrm{C} 4$ & $2.125(3)$ & $\mathrm{C} 33-\mathrm{N} 3$ & $1.181(4)$ \\
$\mathrm{Fe} 1-\mathrm{C} 1$ & $2.172(4)$ & & \\
& & & $177.5(4)$ \\
$\mathrm{C} 33-\mathrm{Fe} 1-\mathrm{C} 24$ & $90.40(14)$ & $\mathrm{C} 15-\mathrm{N} 1-\mathrm{C} 16$ & $174.1(3)$ \\
$\mathrm{C} 33-\mathrm{Fe} 1-\mathrm{C} 15$ & $94.97(14)$ & $\mathrm{C} 24-\mathrm{N} 2-\mathrm{C} 25$ & $166.5(3)$ \\
$\mathrm{C} 24-\mathrm{Fe} 1-\mathrm{C} 15$ & $100.38(14)$ & $\mathrm{C} 33-\mathrm{N} 3-\mathrm{C} 34$ & \\
\hline
\end{tabular}

Table 2

Selected geometric parameters $\left(\AA{ }^{\circ}\right)$ for $\mathbf{2}$.

\begin{tabular}{lclr}
\hline $\mathrm{Fe} 1-\mathrm{C} 10$ & $1.851(3)$ & $\mathrm{Fe} 1-\mathrm{C} 46$ & $1.955(2)$ \\
$\mathrm{Fe} 1-\mathrm{C} 1$ & $1.852(2)$ & $\mathrm{Fe} 1-\mathrm{C} 73$ & $2.011(2)$ \\
$\mathrm{Fe} 1-\mathrm{C} 19$ & $1.854(3)$ & $\mathrm{Fe} 1-\mathrm{C} 28$ & $2.014(2)$ \\
& & & \\
$\mathrm{C} 10-\mathrm{Fe} 1-\mathrm{C} 1$ & $84.67(10)$ & $\mathrm{C} 19-\mathrm{Fe} 1-\mathrm{C} 73$ & $90.39(10)$ \\
$\mathrm{C} 10-\mathrm{Fe} 1-\mathrm{C} 19$ & $94.05(11)$ & $\mathrm{C} 46-\mathrm{Fe} 1-\mathrm{C} 73$ & $81.56(9)$ \\
$\mathrm{C} 1-\mathrm{Fe} 1-\mathrm{C} 19$ & $177.81(11)$ & $\mathrm{C} 10-\mathrm{Fe} 1-\mathrm{C} 28$ & $99.78(10)$ \\
$\mathrm{C} 10-\mathrm{Fe} 1-\mathrm{C} 46$ & $171.45(10)$ & $\mathrm{C} 1-\mathrm{Fe} 1-\mathrm{C} 28$ & $96.36(10)$ \\
$\mathrm{C} 1-\mathrm{Fe} 1-\mathrm{C} 46$ & $86.80(9)$ & $\mathrm{C} 19-\mathrm{Fe} 1-\mathrm{C} 28$ & $85.61(10)$ \\
$\mathrm{C} 19-\mathrm{Fe} 1-\mathrm{C} 46$ & $94.46(10)$ & $\mathrm{C} 46-\mathrm{Fe} 1-\mathrm{C} 28$ & $81.76(10)$ \\
$\mathrm{C} 10-\mathrm{Fe} 1-\mathrm{C} 73$ & $97.51(10)$ & $\mathrm{C} 73-\mathrm{Fe} 1-\mathrm{C} 28$ & $162.48(9)$ \\
$\mathrm{C} 1-\mathrm{Fe} 1-\mathrm{C} 73$ & $88.02(9)$ & & \\
\hline
\end{tabular}

interacting normally with an 18-crown-6 macrocycle, and additionally with the nitrogen atom of the anion (Table 3 ).

\section{Supramolecular features}

In addition to several intermolcular edge-to-face $(\mathrm{C}-\mathrm{H} \cdots \pi)$ interactions, pairs of molecules in $\mathbf{1}$ are linked by offset parallel (slippage, $0.85 \AA$ ) $\pi-\pi$ interactions (Fig. 5), whose centroid-centroid distances are 3.588 (2) $\AA$. In 2 there is one

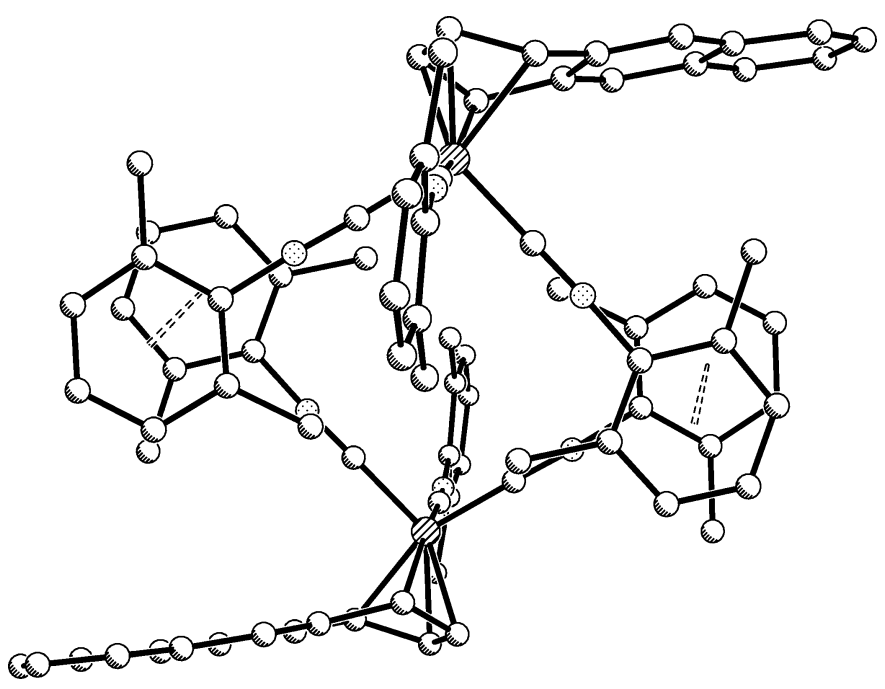

Figure 5

Depiction of the offset parallel $\pi-\pi$ interactions between two molecules of $\mathbf{1}$ whose centroid-centroid (dashed lines) distances are $3.59 \AA$. The second molecule is generated by inversion operator $1-x, 1-y,-z$.
Table 3

Selected bond lengths $(\AA)$ for $\mathbf{3}$.

\begin{tabular}{llll}
\hline $\mathrm{K} 1-\mathrm{N} 1$ & $2.772(3)$ & $\mathrm{K} 1-\mathrm{O} 1$ & $2.835(3)$ \\
$\mathrm{K} 1-\mathrm{O} 5$ & $2.797(2)$ & $\mathrm{K} 1-\mathrm{O} 2$ & $2.846(3)$ \\
$\mathrm{K} 1-\mathrm{O} 4$ & $2.831(3)$ & $\mathrm{K} 1-\mathrm{O} 6$ & $2.959(3)$ \\
$\mathrm{K} 1-\mathrm{O} 3$ & $2.832(3)$ & & \\
\hline
\end{tabular}

Table 4

Hydrogen-bond geometry $\left(\AA,^{\circ}\right)$ for 2 .

\begin{tabular}{lllll}
\hline$D-\mathrm{H} \cdots A$ & $D-\mathrm{H}$ & $\mathrm{H} \cdots A$ & $D \cdots A$ & $D-\mathrm{H} \cdots A$ \\
\hline $\mathrm{N} 8-\mathrm{H} 8 \cdots \mathrm{N} 9$ & 0.86 & 2.10 & $2.515(13)$ & 109 \\
$\mathrm{~N} 8$ - $188^{\prime} \cdots \mathrm{N} 9$ & 0.86 & 2.22 & $2.644(18)$ & 110 \\
\hline
\end{tabular}

instance of an intramolecular offset parallel (slippage, $1.24 \AA$ ) $\pi-\pi$ interaction between phenyl rings C56-C61 and C65-C70 [Fig. 2, centroid-centroid distance, 3.614 (9) ^]. The acceptor for the N7-H7 donor is the $\pi$-system of phenyl ring C47-C52 and that for the N8- H8 donor is intramolecular acceptor N9 (Table 4). No obvious intermolecular interactions are observed in 2, which may also explain the reason for the significant disorder in the thf molecules (i.e., there are no $\mathrm{C}-$ $\mathrm{H}$.. O interactions from the iron complex to anchor them). The intermolecular interactions in $\mathbf{3}$ are limited to $\mathrm{C}-\mathrm{H} \cdots \pi$ interactions between methylene hydrogen atoms and the indenyl $\pi$-system.

\section{Synthesis and crystallization}

All manipulations were carried out under argon using standard Schlenk techniques to maintain strictly anaerobic conditions. Solvents were dried using standard techniques, as described previously (Brennessel \& Ellis, 2012). [K(18-crown6)(THF $\left.)_{2}\right]\left[\mathrm{Fe}\left(\mathrm{C}_{14} \mathrm{H}_{10}\right)_{2}\right]$ and $\mathrm{CNXyl}$ were prepared according to previously reported procedures (Brennessel et al., 2007 and Brennessel et al., 2019, respectively).

To a deep-orange solution of [K(18-crown-6)(thf $\left.)_{2}\right]$ [Fe $\left.\left(\mathrm{C}_{14} \mathrm{H}_{10}\right)_{2}\right](1.000 \mathrm{~g}, 1.163 \mathrm{mmol})$ in thf $(100 \mathrm{~mL}, 195 \mathrm{~K})$ was added CNXyl (1.373 g, $10.47 \mathrm{mmol})$ in thf (40 mL, $195 \mathrm{~K})$. The reaction mixture was warmed slowly to room temperature. A solution IR spectrum showed no anionic species, but a broad peak with shoulders that matched the well-known [Fe(CNXyl) $)_{5}$ (Bassett et al., 1979), as well as a sharp peak for free CNXyl. An aliquot taken early in the reaction was placed in a freezer $(243 \mathrm{~K})$, from which orange crystals of $\mathbf{1}$ were structurally determined. The solvent was removed from the main reaction mixture and heptane was added with vigorous stirring. Crystals grown from the filtrate (i.e., heptane-soluble component) were identified as $\mathbf{2}$ by X-ray diffraction. IR spectroscopy on the crystals (Nujol mull) gave $\nu \mathrm{CN}=2110 \mathrm{w}$ and $2055 \mathrm{vs} \mathrm{cm}^{-1}$. The filter cake (i.e., heptane-insoluble component) was redissolved in THF and layered with pentane, which resulted in crystals of $\mathbf{3}$ as determined by a single crystal $\mathrm{X}$-ray experiment. No characterization beyond what is presented above was performed. 
Table 5

Experimental details.

1

Crystal data

Chemical formula

$M_{\text {r }}$

Crystal system, space group

Temperature $(\mathrm{K})$

$a, b, c(\AA)$

$\alpha, \beta, \gamma\left(^{\circ}\right)$

$V\left(\AA^{3}\right)$

Z

Radiation type

$\mu\left(\mathrm{mm}^{-1}\right)$

Crystal size (mm)

Data collection

Diffractometer

Absorption correction

$T_{\text {min }}, T_{\text {max }}$

No. of measured, independent and observed $[I>2 \sigma(I)]$ reflections

$R_{\text {int }}$

$(\sin \theta / \lambda)_{\max }\left(\AA^{-1}\right)$

Refinement

$R\left[F^{2}>2 \sigma\left(F^{2}\right)\right], w R\left(F^{2}\right), S$

No. of reflections

No. of parameters

No. of restraints

$\mathrm{H}$-atom treatment

$\Delta \rho_{\max }, \Delta \rho_{\min }\left(\mathrm{e} \AA^{-3}\right)$

2

$\left[\mathrm{Fe}\left(\mathrm{C}_{14} \mathrm{H}_{10}\right)\left(\mathrm{C}_{9} \mathrm{H}_{9} \mathrm{~N}\right)_{3}\right]$
627.58
Monoclinic, $P 2_{1} / n$
$\quad 173$
$11.8528(11), 10.9022(10)$,
$\quad 24.927(2)$
$90,93.057(2), 90$
$3216.5(5)$
4
$\mathrm{Mo} \mathrm{K \alpha}$
0.50
$0.23 \times 0.14 \times 0.08$

Siemens SMART CCD platform Multi-scan ( $S A D A B S$; Krause et al., 2015)

$0.820,1.000$

24459, 5687, 4023

0.087

0.596

$0.060,0.120,1.05$
5687
429
0
H atoms treated by a mixture of
$\quad$ independent and constrained
$\quad$ refinement
$0.36,-0.35$

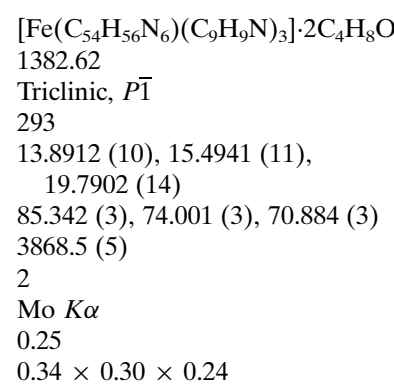

Bruker SMART CCD platform

Multi-scan (SADABS; Krause et al., 2015)

$0.925,1.000$

31905, 13640, 9367

0.040

0.596

$0.051,0.136,1.02$

13640

1192

382

$\mathrm{H}$ atoms treated by a mixture of independent and constrained refinement

$0.38,-0.18$
3

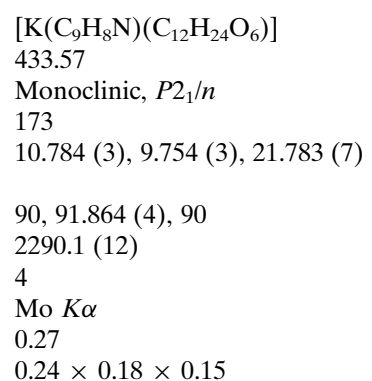

Bruker SMART CCD platform Multi-scan ( $S A D A B S$; Krause et al., 2015)

$0.843,1.000$

21112, 4071, 2902

0.062

0.596

$0.059,0.170,1.05$
4071
355
195
H-atom parameters constrained

$0.58,-0.23$

Computer programs: SMART and SAINT (Bruker, 2003), SIR97 (Altomare et al., 1999), SHELXL2018/3 (Sheldrick, 2015), and SHELXTL (Sheldrick, 2008).

\section{Refinement}

Crystal data, data collection and structure refinement details are summarized in Table 5. Intensity data for $\mathbf{2}$ were collected at 293 (3) K. A preliminary collection at 173 (2) K resulted in a primitive monoclinic cell which was modulated such that the $b$-axis was doubled and the $a$-axis could be determined as multiples of approximately $13 \AA$, the best being $52 \AA$.

In 2, two CNXyl groups were modeled as disordered over two positions each: N1/C1-C9, 0.52 (2):0.48 (2) and N8/C64C72, 0.57 (2):0.43 (2). Additionally, the two THF solvent molecules were modeled as disordered over two positions each: O1/C82-C85, 0.55 (2):0.45 (2) and O2/C86-C89, 0.69 (1):0.31 (1).

In $\mathbf{3}$, the anion is modeled as disordered with a planar flip of itself [0.905 (3):0.095 (3)]. The 18-crown-6 macrocycle is also disordered in a similarly lopsided component ratio; the eight largest residual peaks are the two peaks near the $\mathrm{K}$ atom and those for six $\mathrm{O}$ atoms of the minor component of disorder. However, the data-to-parameter ratio drops below eight if this disorder is modeled. Thus only the anion disorder was modeled.

To model the various disordered species, analogous bond lengths and angles were restrained to be similar and anisotropic displacement parameters for proximal atoms were restrained to be similar. For the THF solvent molecules in $\mathbf{2}$, bond lengths were restrained toward ideal values and anisotropic displacement parameters were additionally restrained toward the expected motion relative to bond direction.

The $\mathrm{H}$ atoms on the metal-coordinating carbon atoms $(\mathrm{C} 1-$ $\mathrm{C} 4)$ of 1 were refined freely to confirm their nature and better describe their true positions. In $\mathbf{2}, \mathrm{H} 7$ was also refined freely. All other $\mathrm{H}$ atoms were placed geometrically and treated as riding atoms. For $\mathbf{1}$ and $\mathbf{3}(173 \mathrm{~K})$, methylene, $\mathrm{C}-\mathrm{H}=0.99 \AA$, aromatic/sp $p^{2}, \mathrm{C}-\mathrm{H}=0.95 \AA$, with $U_{\text {iso }}(\mathrm{H})=1.2 U_{\mathrm{eq}}(\mathrm{C})$, and methyl, $\mathrm{C}-\mathrm{H}=0.98 \AA$, with $U_{\text {iso }}(\mathrm{H})=1.5 U_{\text {eq }}(\mathrm{C})$. For 2 $(293 \mathrm{~K})$, methylene, $\mathrm{C}-\mathrm{H}=0.97 \AA$, aromatic $/ s p^{2}, \mathrm{C}-\mathrm{H}=$ $0.93 \AA, \mathrm{N}-\mathrm{H}=0.86 \AA$, with $U_{\text {iso }}(\mathrm{H})=1.2 U_{\text {eq }}(\mathrm{C})$, and methyl, $\mathrm{C}-\mathrm{H}=0.96 \AA$, with $U_{\text {iso }}(\mathrm{H})=1.5 U_{\text {eq }}(\mathrm{C})$.

For 1 the maximum residual peak of $0.36 \mathrm{e}^{-} \AA^{-3}$ and the deepest hole of $-0.35 \mathrm{e}^{-} \AA^{-3}$ are found 0.97 and $0.53 \AA$ from atoms $\mathrm{C} 2$ and $\mathrm{Fe} 1$, respectively.

For 2 the maximum residual peak of $0.38 \mathrm{e}^{-} \AA^{-3}$ and the deepest hole of $-0.18 \mathrm{e}^{-} \AA^{-3}$ are found 0.81 and $0.39 \AA$ from atoms $\mathrm{H} 15$ and $\mathrm{C} 14$, respectively.

For 3 the maximum residual peak of $0.58 \mathrm{e}^{-} \AA^{-3}$ and the deepest hole of $-0.23 \mathrm{e}^{-} \AA^{-3}$ are found 1.15 and $1.25 \AA$ from atoms $\mathrm{C} 15$ and $\mathrm{K} 1$, respectively. The peak is part of the minor component of disorder of the 18-crown-6 ring, which was not modeled (see above). 


\section{Acknowledgements}

The authors thank Dr Victor G. Young, Jr and Margaret L. Clapham of the X-ray Crystallographic Laboratory of the University of Minnesota Chemistry Department for use of the instrumentation and for assistance with data retrieval.

\section{Funding information}

The authors acknowledge the US National Science Foundation, and the donors of the Petroleum Research Fund, administered by the American Chemical Society, for funding.

\section{References}

Altenburger, K., Arndt, P., Becker, L., Reiss, F., Burlakov, V. V., Spannenberg, A., Baumann, W. \& Rosenthal, U. (2016). Chem. Eur. J. 22, 9169-9180.

Altomare, A., Burla, M. C., Camalli, M., Cascarano, G. L., Giacovazzo, C., Guagliardi, A., Moliterni, A. G. G., Polidori, G. \& Spagna, R. (1999). J. Appl. Cryst. 32, 115-119.

Bashall, A., Collier, P., Gade, L. H., McPartlin, M., Mountford, P., Pugh, S. M., Radojevic, S., Schubart, M., Scowen, I. J. \& Trösch, J. M. (2000). Organometallics, 19, 4784-4794.

Bassett, J.-M., Berry, D. E., Barker, G. K., Green, M., Howard, J. A. K. \& Stone, F. G. A. (1979). J. Chem. Soc. Dalton Trans. pp. 10031011.

Blake, A. J., Collier, P. E., Gade, L. H., McPartlin, M., Mountford, P., Schubart, M. \& Scowen, I. J. (1997). Chem. Commun. pp. 15551556.

Brennessel, W. W. \& Ellis, J. E. (2007). Angew. Chem. Int. Ed. 46, 598600.

Brennessel, W. W. \& Ellis, J. E. (2012). Inorg. Chem. 51, 9076-9094.

Brennessel, W. W., Jilek, R. E. \& Ellis, J. E. (2007). Angew. Chem. Int. Ed. 46, 6132-6136.
Brennessel, W. W., Kucera, B. E., Young, V. G. Jr \& Ellis, J. E. (2019). Acta Cryst. C75, 1118-1127.

Brennessel, W. W., Young, V. G. Jr \& Ellis, J. E. (2002). Angew. Chem. Int. Ed. 41, 1211-1215.

Bruker (2003). SAINT and SMART. Bruker AXS, Inc., Madison, Wisconsin, USA.

Chen, W., Zhao, Y., Xu, W., Su, J.-H., Shen, L., Liu, L., Wu, B. \& Yang, X.-J. (2019). Chem. Commun. 55, 9452-9455.

Giandomenico, C. M., Lam, C. T. \& Lippard, S. J. (1982). J. Am. Chem. Soc. 104, 1263-1271.

Imhof, W. (1999). Organometallics, 18, 4845-4855.

Krause, L., Herbst-Irmer, R., Sheldrick, G. M. \& Stalke, D. (2015). J. Appl. Cryst. 48, 3-10.

Kucera, B. E., Roberts, C. J., Young, V. G., Brennessel, W. W. \& Ellis, J. E. (2019). Acta Cryst. C75, 1259-1265.

Lam, C. T., Corfield, P. W. R. \& Lippard, S. J. (1977). J. Am. Chem. Soc. 99, 617-618.

Manuel, T. A. (1964). Inorg. Chem. 3, 1794-1796.

Schäufele, H., Hu, D., Pritzkow, H. \& Zenneck, U. (1989). Organometallics, 8, 396-401.

Sheldrick, G. M. (2008). Acta Cryst. A64, 112-122.

Sheldrick, G. M. (2015). Acta Cryst. C71, 3-8.

Shen, J., Yap, G. P. A. \& Theopold, K. H. (2014). J. Am. Chem. Soc. 136, 3382-3384.

Tanase, T., Ohizumi, T., Kobayashi, K. \& Yamamoto, Y. (1992). J. Chem. Soc. Chem. Commun. pp. 707-708.

Tanase, T., Ohizumi, T., Kobayashi, K. \& Yamamoto, Y. (1996). Organometallics, 15, 3404-3411.

Warner, S. \& Lippard, S. J. (1986). Organometallics, 5, 1716-1725.

Warnock, G. F. \& Cooper, N. J. (1989). Organometallics, 8, 1826-1827.

Yamamoto, Y. \& Yamazaki, H. (1972). Coord. Chem. Rev. 8, 225-239.

Yamamoto, Y., Yamazaki, H. \& Sakurai, T. (1982). J. Am. Chem. Soc. 104, 2329-2330. 


\section{supporting information}

Acta Cryst. (2022). E78, 60-65 [https://doi.org/10.1107/S205698902101313X]

\section{Crystal structures of two novel iron isocyanides from the reaction of 2,6-di- methylphenyl isocyanide, $\mathrm{CNXYl}$, with bis(anthracene)ferrate(-1)}

\section{William W. Brennessel and John E. Ellis}

\section{Computing details}

For all structures, data collection: SMART (Bruker, 2003); cell refinement: SAINT (Bruker, 2003); data reduction: SAINT (Bruker, 2003); program(s) used to solve structure: SIR97 (Altomare et al., 1999); program(s) used to refine structure: SHELXL2018/3 (Sheldrick, 2015); molecular graphics: SHELXTL (Sheldrick, 2008); software used to prepare material for publication: SHELXTL (Sheldrick, 2008).

$[(1,2,3,4-\eta)$-Anthracene]tris(2,6-dimethylphenyl isocyanide)iron (1)

\section{Crystal data}

$\left[\mathrm{Fe}\left(\mathrm{C}_{14} \mathrm{H}_{10}\right)\left(\mathrm{C}_{9} \mathrm{H}_{9} \mathrm{~N}\right)_{3}\right]$

$M_{r}=627.58$

Monoclinic, $P 2{ }_{1} / n$

$a=11.8528(11) \AA$

$b=10.9022(10) \AA$

$c=24.927(2) \AA$

$\beta=93.057(2)^{\circ}$

$V=3216.5(5) \AA^{3}$

$Z=4$

\section{Data collection}

Siemens SMART CCD platform diffractometer

Radiation source: normal-focus sealed tube $\omega$ scans

Absorption correction: multi-scan

(SADABS; Krause et al., 2015)

$T_{\min }=0.820, T_{\max }=1.000$

24459 measured reflections

\section{Refinement}

Refinement on $F^{2}$

Least-squares matrix: full

$R\left[F^{2}>2 \sigma\left(F^{2}\right)\right]=0.060$

$w R\left(F^{2}\right)=0.120$

$S=1.05$

5687 reflections

429 parameters

0 restraints

Primary atom site location: structure-invariant direct methods
$F(000)=1320$

$D_{\mathrm{x}}=1.296 \mathrm{Mg} \mathrm{m}^{-3}$

Mo $K \alpha$ radiation, $\lambda=0.71073 \AA$

Cell parameters from 2566 reflections

$\theta=2.5-24.3^{\circ}$

$\mu=0.50 \mathrm{~mm}^{-1}$

$T=173 \mathrm{~K}$

Block, orange

$0.23 \times 0.14 \times 0.08 \mathrm{~mm}$

5687 independent reflections

4023 reflections with $I>2 \sigma(I)$

$R_{\text {int }}=0.087$

$\theta_{\text {max }}=25.1^{\circ}, \theta_{\min }=1.6^{\circ}$

$h=-14 \rightarrow 13$

$k=-12 \rightarrow 12$

$l=-29 \rightarrow 29$

Secondary atom site location: difference Fourier map

Hydrogen site location: mixed

$\mathrm{H}$ atoms treated by a mixture of independent and constrained refinement

$w=1 /\left[\sigma^{2}\left(F_{\mathrm{o}}^{2}\right)+(0.0505 P)^{2}+1.1491 P\right]$

$$
\text { where } P=\left(F_{\mathrm{o}}^{2}+2 F_{\mathrm{c}}{ }^{2}\right) / 3
$$

$(\Delta / \sigma)_{\max }<0.001$

$\Delta \rho_{\max }=0.36$ e $\AA^{-3}$

$\Delta \rho_{\min }=-0.35$ e $\AA^{-3}$ 
Extinction correction: SHELXL-2018/3

(Sheldrick 2015),

$\mathrm{Fc}^{*}=\mathrm{kFc}\left[1+0.001 \times \mathrm{Fc}^{2} \lambda^{3} / \sin (2 \theta)\right]^{-1 / 4}$

Extinction coefficient: 0.0108 (7)

\section{Special details}

Geometry. All esds (except the esd in the dihedral angle between two 1.s. planes) are estimated using the full covariance matrix. The cell esds are taken into account individually in the estimation of esds in distances, angles and torsion angles; correlations between esds in cell parameters are only used when they are defined by crystal symmetry. An approximate (isotropic) treatment of cell esds is used for estimating esds involving l.s. planes.

Fractional atomic coordinates and isotropic or equivalent isotropic displacement parameters $\left(\AA^{2}\right)$

\begin{tabular}{|c|c|c|c|c|}
\hline & $x$ & $y$ & $z$ & $U_{\text {iso }} * / U_{\text {eq }}$ \\
\hline $\mathrm{Fe} 1$ & $0.31214(4)$ & $0.23419(4)$ & $0.06206(2)$ & $0.02761(16)$ \\
\hline $\mathrm{C} 1$ & $0.3597(3)$ & $0.1374(3)$ & $-0.00948(15)$ & $0.0344(9)$ \\
\hline $\mathrm{H} 1 \mathrm{~A}$ & $0.427(3)$ & $0.157(3)$ & $-0.0271(14)$ & $0.049(11)^{*}$ \\
\hline $\mathrm{C} 2$ & $0.3697(3)$ & $0.0671(3)$ & $0.03826(16)$ & $0.0378(9)$ \\
\hline $\mathrm{H} 2 \mathrm{~A}$ & $0.437(3)$ & $0.038(3)$ & $0.0535(14)$ & $0.040(10)^{*}$ \\
\hline $\mathrm{C} 3$ & $0.2706(3)$ & $0.0535(3)$ & $0.06579(16)$ & $0.0346(9)$ \\
\hline $\mathrm{H} 3 \mathrm{~A}$ & $0.267(3)$ & $0.007(3)$ & $0.0977(15)$ & $0.053(12)^{*}$ \\
\hline $\mathrm{C} 4$ & $0.1740(3)$ & $0.1151(3)$ & $0.04281(14)$ & $0.0286(8)$ \\
\hline $\mathrm{H} 4 \mathrm{~A}$ & $0.113(3)$ & $0.116(3)$ & $0.0631(13)$ & $0.032(9)^{*}$ \\
\hline $\mathrm{C} 5$ & $-0.0638(3)$ & $0.1476(3)$ & $-0.12786(15)$ & $0.0408(9)$ \\
\hline $\mathrm{H} 5 \mathrm{~A}$ & -0.131036 & 0.146436 & -0.108779 & $0.049^{*}$ \\
\hline C6 & $-0.0713(4)$ & $0.1606(3)$ & $-0.18278(16)$ & $0.0502(11)$ \\
\hline H6A & -0.143118 & 0.167987 & -0.201270 & $0.060 *$ \\
\hline $\mathrm{C} 7$ & $0.0268(4)$ & $0.1628(3)$ & $-0.21135(16)$ & $0.0549(12)$ \\
\hline H7A & 0.021654 & 0.171483 & -0.249322 & $0.066^{*}$ \\
\hline $\mathrm{C} 8$ & $0.1300(4)$ & $0.1527(3)$ & $-0.18487(15)$ & $0.0493(11)$ \\
\hline H8A & 0.195912 & 0.154133 & -0.204906 & $0.059^{*}$ \\
\hline C9 & $0.2481(3)$ & $0.1367(3)$ & $-0.09923(15)$ & $0.0381(9)$ \\
\hline H9A & 0.315408 & 0.138178 & -0.118294 & $0.046^{*}$ \\
\hline $\mathrm{C} 10$ & $0.0513(3)$ & $0.1235(3)$ & $-0.04268(13)$ & $0.0297(8)$ \\
\hline $\mathrm{H} 10 \mathrm{~A}$ & -0.015070 & 0.117228 & -0.023102 & $0.036^{*}$ \\
\hline C11 & $0.2555(3)$ & $0.1312(3)$ & $-0.04438(14)$ & $0.0313(8)$ \\
\hline $\mathrm{C} 12$ & $0.1552(3)$ & $0.1205(3)$ & $-0.01571(13)$ & $0.0276(8)$ \\
\hline $\mathrm{C} 13$ & $0.0415(3)$ & $0.1359(3)$ & $-0.09917(13)$ & $0.0330(8)$ \\
\hline $\mathrm{C} 14$ & $0.1416(3)$ & $0.1402(3)$ & $-0.12837(14)$ & $0.0348(9)$ \\
\hline $\mathrm{C} 15$ & $0.2630(3)$ & $0.3741(3)$ & $0.02621(14)$ & $0.0301(8)$ \\
\hline N1 & $0.2304(2)$ & $0.4627(2)$ & $0.00412(12)$ & $0.0356(7)$ \\
\hline $\mathrm{C} 16$ & $0.1942(3)$ & $0.5721(3)$ & $-0.02091(13)$ & $0.0299(8)$ \\
\hline $\mathrm{C} 17$ & $0.1030(3)$ & $0.5683(3)$ & $-0.05881(13)$ & $0.0323(8)$ \\
\hline $\mathrm{C} 18$ & $0.0705(3)$ & $0.6776(3)$ & $-0.08270(14)$ & $0.0377(9)$ \\
\hline H18A & 0.008746 & 0.678274 & -0.108631 & $0.045^{*}$ \\
\hline C19 & $0.1257(3)$ & $0.7863(3)$ & $-0.06976(14)$ & $0.0389(9)$ \\
\hline H19A & 0.101896 & 0.860406 & -0.086943 & $0.047^{*}$ \\
\hline $\mathrm{C} 20$ & $0.2143(3)$ & $0.7875(3)$ & $-0.03237(14)$ & $0.0363(8)$ \\
\hline $\mathrm{H} 20 \mathrm{~A}$ & 0.251753 & 0.862681 & -0.023879 & $0.044 *$ \\
\hline
\end{tabular}




\begin{tabular}{|c|c|c|c|c|}
\hline $\mathrm{C} 21$ & $0.2506(3)$ & $0.6801(3)$ & $-0.00653(13)$ & $0.0320(8)$ \\
\hline $\mathrm{C} 22$ & 0.0448 & $0.4489(3)$ & $-0.07298(16)$ & $0.0487(10)$ \\
\hline $\mathrm{H} 22 \mathrm{~A}$ & -0.015053 & 0.463279 & -0.100939 & $0.073^{*}$ \\
\hline $\mathrm{H} 22 \mathrm{~B}$ & 0.011639 & 0.414999 & -0.040972 & $0.073^{*}$ \\
\hline $\mathrm{H} 22 \mathrm{C}$ & 0.099809 & 0.390683 & -0.086233 & $0.073^{*}$ \\
\hline $\mathrm{C} 23$ & 0.3474 & $0.6811(4)$ & $0.03499(17)$ & $0.0537(11)$ \\
\hline $\mathrm{H} 23 \mathrm{~A}$ & 0.327591 & 0.632025 & 0.066055 & $0.081^{*}$ \\
\hline $\mathrm{H} 23 \mathrm{~B}$ & 0.363206 & 0.765629 & 0.046433 & $0.081^{*}$ \\
\hline $\mathrm{H} 23 \mathrm{C}$ & 0.414650 & 0.646176 & 0.019532 & $0.081^{*}$ \\
\hline $\mathrm{C} 24$ & $0.4511(3)$ & $0.2860(3)$ & $0.08974(14)$ & $0.0316(8)$ \\
\hline N2 & $0.5361(2)$ & $0.3168(3)$ & $0.11121(12)$ & $0.0385(7)$ \\
\hline $\mathrm{C} 25$ & $0.6315(3)$ & $0.3606(3)$ & $0.14059(13)$ & $0.0296(8)$ \\
\hline $\mathrm{C} 26$ & 0.6539 & $0.4863(3)$ & $0.13917(14)$ & $0.0340(8)$ \\
\hline $\mathrm{C} 27$ & 0.7473 & $0.5284(3)$ & $0.16943(15)$ & $0.0426(9)$ \\
\hline $\mathrm{H} 27 \mathrm{~A}$ & 0.764292 & 0.613569 & 0.169569 & $0.051^{*}$ \\
\hline C28 & $0.8156(3)$ & 0.4497 (4) & $0.19920(15)$ & $0.0469(10)$ \\
\hline $\mathrm{H} 28 \mathrm{~A}$ & 0.879716 & 0.480378 & 0.219426 & $0.056^{*}$ \\
\hline $\mathrm{C} 29$ & $0.7912(3)$ & $0.3261(4)$ & $0.19977(14)$ & $0.0427(9)$ \\
\hline $\mathrm{H} 29 \mathrm{~A}$ & 0.838982 & 0.272388 & 0.220648 & $0.051^{*}$ \\
\hline C 30 & $0.6986(3)$ & $0.2783(3)$ & $0.17056(14)$ & $0.0348(8)$ \\
\hline $\mathrm{C} 31$ & 0.5800 & $0.5698(3)$ & $0.10484(17)$ & 0.0539 (11) \\
\hline $\mathrm{H} 31 \mathrm{~A}$ & 0.575586 & 0.539425 & 0.067785 & $0.081 *$ \\
\hline $\mathrm{H} 31 \mathrm{~B}$ & 0.612114 & 0.652662 & 0.105700 & $0.081^{*}$ \\
\hline $\mathrm{H} 31 \mathrm{C}$ & 0.504147 & 0.571857 & 0.118604 & $0.081 *$ \\
\hline $\mathrm{C} 32$ & $0.6720(4)$ & $0.1442(3)$ & $0.17089(18)$ & $0.0558(12)$ \\
\hline $\mathrm{H} 32 \mathrm{~A}$ & 0.731368 & 0.100511 & 0.192037 & $0.084 *$ \\
\hline $\mathrm{H} 32 \mathrm{~B}$ & 0.668099 & 0.113055 & 0.133959 & $0.084 *$ \\
\hline $\mathrm{H} 32 \mathrm{C}$ & 0.599212 & 0.131257 & 0.186880 & $0.084 *$ \\
\hline C33 & $0.2496(3)$ & $0.2732(3)$ & $0.12404(14)$ & $0.0316(8)$ \\
\hline N3 & $0.2010(2)$ & $0.2945(3)$ & $0.16316(12)$ & $0.0386(7)$ \\
\hline C34 & $0.1558(3)$ & $0.3463(3)$ & $0.20781(14)$ & $0.0322(8)$ \\
\hline C35 & $0.1799(3)$ & 0.4689 (3) & $0.21915(15)$ & $0.0415(9)$ \\
\hline $\mathrm{C} 36$ & 0.1398 & $0.5169(4)$ & $0.26596(18)$ & $0.0538(11)$ \\
\hline $\mathrm{H} 36 \mathrm{~A}$ & 0.154918 & 0.600200 & 0.274916 & $0.065^{*}$ \\
\hline C37 & $0.0787(3)$ & $0.4468(4)$ & 0.29957 (17) & 0.0585 (13) \\
\hline H37A & 0.054290 & 0.480930 & 0.332048 & $0.070^{*}$ \\
\hline C38 & $0.0521(3)$ & 0.3257 (4) & $0.28640(16)$ & $0.0496(10)$ \\
\hline $\mathrm{H} 38 \mathrm{~A}$ & 0.008566 & 0.278127 & 0.309637 & $0.060^{*}$ \\
\hline C39 & 0.0888 & $0.2741(3)$ & $0.23960(14)$ & $0.0365(8)$ \\
\hline $\mathrm{C} 40$ & $0.2463(4)$ & $0.5432(4)$ & $0.18151(18)$ & 0.0639 (13) \\
\hline $\mathrm{H} 40 \mathrm{~B}$ & 0.251798 & 0.627969 & 0.194415 & $0.096^{*}$ \\
\hline $\mathrm{H} 40 \mathrm{C}$ & 0.208241 & 0.541810 & 0.145624 & $0.096^{*}$ \\
\hline $\mathrm{H} 40 \mathrm{D}$ & 0.322229 & 0.508409 & 0.179802 & $0.096^{*}$ \\
\hline C41 & $0.0569(3)$ & 0.1459 (3) & $0.22234(17)$ & $0.0484(10)$ \\
\hline $\mathrm{H} 41 \mathrm{~B}$ & 0.125365 & 0.099304 & 0.215470 & $0.073^{*}$ \\
\hline $\mathrm{H} 41 \mathrm{C}$ & 0.007835 & 0.149243 & 0.189459 & $0.073^{*}$ \\
\hline H41D & 0.016726 & 0.105596 & 0.250836 & $0.073 *$ \\
\hline
\end{tabular}


Atomic displacement parameters $\left(\AA^{2}\right)$

\begin{tabular}{|c|c|c|c|c|c|c|}
\hline & $U^{11}$ & $U^{22}$ & $U^{33}$ & $U^{12}$ & $U^{13}$ & $U^{23}$ \\
\hline $\mathrm{Fe} 1$ & 0.0248 & $0.0193(2)$ & $0.0384(3)$ & $-0.0033(2)$ & $-0.0010(2)$ & $-0.0014(2)$ \\
\hline $\mathrm{C} 1$ & $0.030(2)$ & $0.0243(17)$ & $0.050(2)$ & $-0.0045(15)$ & $0.0110(19)$ & $-0.0073(16)$ \\
\hline $\mathrm{C} 2$ & $0.034(2)$ & $0.0200(17)$ & $0.058(3)$ & $0.0009(15)$ & $-0.005(2)$ & $-0.0062(17)$ \\
\hline $\mathrm{C} 3$ & $0.039(2)$ & $0.0187(17)$ & $0.046(2)$ & $-0.0078(14)$ & $-0.0027(19)$ & $0.0000(16)$ \\
\hline $\mathrm{C} 4$ & $0.028(2)$ & $0.0227(17)$ & $0.035(2)$ & $-0.0071(14)$ & $0.0047(17)$ & $0.0002(14)$ \\
\hline $\mathrm{C} 5$ & $0.050(2)$ & $0.0282(19)$ & $0.044(2)$ & $-0.0124(16)$ & $-0.003(2)$ & $0.0019(16)$ \\
\hline C6 & $0.071(3)$ & $0.036(2)$ & $0.042(2)$ & $-0.0143(19)$ & $-0.014(2)$ & $0.0046(18)$ \\
\hline $\mathrm{C} 7$ & $0.092(4)$ & $0.038(2)$ & $0.033(2)$ & $-0.020(2)$ & $-0.004(3)$ & $-0.0010(18)$ \\
\hline $\mathrm{C} 8$ & $0.076(3)$ & $0.033(2)$ & $0.041(2)$ & $-0.015(2)$ & $0.020(2)$ & $-0.0076(17)$ \\
\hline C9 & $0.043(2)$ & $0.0262(18)$ & $0.046(2)$ & $-0.0077(16)$ & 0.0167 (19) & $-0.0104(16)$ \\
\hline $\mathrm{C} 10$ & $0.034(2)$ & $0.0229(17)$ & $0.0324(19)$ & $-0.0107(14)$ & $0.0061(16)$ & $-0.0002(14)$ \\
\hline C11 & $0.033(2)$ & $0.0197(16)$ & $0.042(2)$ & $-0.0054(14)$ & $0.0088(17)$ & $-0.0046(14)$ \\
\hline $\mathrm{C} 12$ & $0.032(2)$ & $0.0161(15)$ & $0.035(2)$ & $-0.0077(13)$ & $0.0047(16)$ & $-0.0042(13)$ \\
\hline $\mathrm{C} 13$ & $0.046(2)$ & $0.0202(16)$ & $0.033(2)$ & $-0.0109(15)$ & $0.0022(18)$ & $-0.0021(14)$ \\
\hline $\mathrm{C} 14$ & $0.054(2)$ & $0.0183(16)$ & $0.032(2)$ & $-0.0130(15)$ & $0.0089(18)$ & $-0.0047(14)$ \\
\hline $\mathrm{C} 15$ & 0.0269 (19) & $0.0263(18)$ & $0.037(2)$ & $-0.0082(14)$ & $0.0034(16)$ & $-0.0060(16)$ \\
\hline N1 & $0.0382(17)$ & $0.0258(16)$ & $0.0432(18)$ & $0.0025(13)$ & $0.0062(14)$ & $0.0059(13)$ \\
\hline C16 & 0.0307 (19) & $0.0235(17)$ & $0.036(2)$ & $0.0025(14)$ & $0.0096(16)$ & $0.0030(14)$ \\
\hline $\mathrm{C} 17$ & 0.0299 (19) & $0.0338(19)$ & $0.034(2)$ & $-0.0008(15)$ & $0.0065(16)$ & $-0.0029(15)$ \\
\hline $\mathrm{C} 18$ & $0.033(2)$ & $0.045(2)$ & $0.035(2)$ & $0.0029(17)$ & 0.0002 (17) & $-0.0013(17)$ \\
\hline C19 & $0.049(2)$ & $0.029(2)$ & $0.039(2)$ & $0.0106(16)$ & 0.0089 (19) & $0.0046(16)$ \\
\hline $\mathrm{C} 20$ & $0.044(2)$ & 0.0275 (19) & $0.037(2)$ & $-0.0039(15)$ & 0.0065 (18) & $-0.0032(15)$ \\
\hline $\mathrm{C} 21$ & $0.032(2)$ & $0.0301(18)$ & 0.0338 (19) & $-0.0009(15)$ & $0.0027(16)$ & $-0.0007(15)$ \\
\hline $\mathrm{C} 22$ & $0.050(2)$ & $0.042(2)$ & $0.054(3)$ & $-0.0151(18)$ & $0.000(2)$ & -0.0075 (19) \\
\hline $\mathrm{C} 23$ & $0.047(2)$ & $0.055(2)$ & $0.057(3)$ & $-0.011(2)$ & -0.009 (2) & $0.002(2)$ \\
\hline $\mathrm{C} 24$ & 0.0312 (19) & $0.0214(17)$ & $0.043(2)$ & $0.0012(14)$ & $0.0041(17)$ & $-0.0005(15)$ \\
\hline N2 & $0.0294(17)$ & $0.0393(17)$ & 0.0463 (19) & $-0.0059(14)$ & $-0.0011(15)$ & $-0.0091(14)$ \\
\hline $\mathrm{C} 25$ & $0.0219(18)$ & $0.0326(18)$ & 0.0344 (19) & $-0.0039(14)$ & $0.0021(15)$ & $-0.0050(15)$ \\
\hline $\mathrm{C} 26$ & $0.035(2)$ & 0.0324 (19) & $0.035(2)$ & $-0.0044(15)$ & $0.0035(16)$ & $-0.0024(15)$ \\
\hline $\mathrm{C} 27$ & $0.043(2)$ & $0.038(2)$ & $0.047(2)$ & $-0.0148(18)$ & $0.006(2)$ & $-0.0104(18)$ \\
\hline $\mathrm{C} 28$ & $0.033(2)$ & $0.069(3)$ & $0.038(2)$ & $-0.011(2)$ & 0.0007 (18) & $-0.020(2)$ \\
\hline $\mathrm{C} 29$ & $0.033(2)$ & $0.060(3)$ & $0.035(2)$ & 0.0077 (18) & $-0.0001(18)$ & $-0.0004(18)$ \\
\hline $\mathrm{C} 30$ & 0.0337 (19) & $0.0333(18)$ & $0.038(2)$ & $0.0005(15)$ & $0.0071(16)$ & $-0.0003(16)$ \\
\hline C31 & $0.053(3)$ & $0.040(2)$ & $0.069(3)$ & 0.0018 (19) & $-0.003(2)$ & $0.007(2)$ \\
\hline $\mathrm{C} 32$ & $0.058(3)$ & $0.035(2)$ & $0.074(3)$ & 0.0019 (19) & $0.008(2)$ & $0.011(2)$ \\
\hline C33 & $0.0275(18)$ & $0.0254(17)$ & $0.041(2)$ & $-0.0044(14)$ & $-0.0090(17)$ & $-0.0011(16)$ \\
\hline N3 & $0.0342(17)$ & $0.0408(18)$ & 0.0401 (19) & $-0.0038(13)$ & $-0.0034(15)$ & $-0.0076(14)$ \\
\hline $\mathrm{C} 34$ & $0.0280(19)$ & 0.0350 (19) & $0.033(2)$ & $0.0043(15)$ & $-0.0060(16)$ & $-0.0070(16)$ \\
\hline $\mathrm{C} 35$ & $0.034(2)$ & $0.042(2)$ & $0.047(2)$ & 0.0007 (17) & $-0.0158(18)$ & $-0.0085(18)$ \\
\hline $\mathrm{C} 36$ & $0.046(3)$ & $0.050(2)$ & $0.063(3)$ & $0.012(2)$ & $-0.018(2)$ & $-0.025(2)$ \\
\hline $\mathrm{C} 37$ & $0.042(3)$ & $0.085(3)$ & $0.047(3)$ & $0.027(2)$ & $-0.009(2)$ & $-0.027(2)$ \\
\hline C38 & $0.036(2)$ & $0.070(3)$ & $0.042(2)$ & $0.013(2)$ & -0.0038 (19) & $0.002(2)$ \\
\hline C39 & 0.0307 (19) & 0.039 (2) & $0.039(2)$ & $0.0108(16)$ & $-0.0059(16)$ & $0.0011(17)$ \\
\hline $\mathrm{C} 40$ & $0.068(3)$ & $0.049(3)$ & $0.073(3)$ & $-0.018(2)$ & -0.017 & $0.005(2)$ \\
\hline $\mathrm{C} 41$ & $0.043(2)$ & $0.042(2)$ & $0.060(3)$ & $0.0004(18)$ & $0.000(2)$ & 0.0053 (19) \\
\hline
\end{tabular}


Geometric parameters $\left(\AA,{ }^{\circ}\right)$

\begin{tabular}{|c|c|c|c|}
\hline $\mathrm{Fe} 1-\mathrm{C} 33$ & $1.800(4)$ & $\mathrm{C} 21-\mathrm{C} 23$ & $1.504(5)$ \\
\hline $\mathrm{Fe} 1-\mathrm{C} 24$ & $1.840(3)$ & $\mathrm{C} 22-\mathrm{H} 22 \mathrm{~A}$ & 0.9800 \\
\hline $\mathrm{Fe} 1-\mathrm{C} 15$ & $1.847(3)$ & $\mathrm{C} 22-\mathrm{H} 22 \mathrm{~B}$ & 0.9800 \\
\hline $\mathrm{Fe} 1-\mathrm{C} 3$ & $2.034(3)$ & $\mathrm{C} 22-\mathrm{H} 22 \mathrm{C}$ & 0.9800 \\
\hline $\mathrm{Fe} 1-\mathrm{C} 2$ & $2.044(3)$ & $\mathrm{C} 23-\mathrm{H} 23 \mathrm{~A}$ & 0.9800 \\
\hline $\mathrm{Fe} 1-\mathrm{C} 4$ & $2.125(3)$ & $\mathrm{C} 23-\mathrm{H} 23 \mathrm{~B}$ & 0.9800 \\
\hline $\mathrm{Fe} 1-\mathrm{C} 1$ & $2.172(4)$ & $\mathrm{C} 23-\mathrm{H} 23 \mathrm{C}$ & 0.9800 \\
\hline $\mathrm{C} 1-\mathrm{C} 2$ & $1.415(5)$ & $\mathrm{C} 24-\mathrm{N} 2$ & $1.165(4)$ \\
\hline $\mathrm{C} 1-\mathrm{C} 11$ & $1.473(5)$ & $\mathrm{N} 2-\mathrm{C} 25$ & $1.398(4)$ \\
\hline $\mathrm{C} 1-\mathrm{H} 1 \mathrm{~A}$ & $0.96(4)$ & $\mathrm{C} 25-\mathrm{C} 30$ & $1.389(4)$ \\
\hline $\mathrm{C} 2-\mathrm{C} 3$ & $1.399(5)$ & $\mathrm{C} 25-\mathrm{C} 26$ & $1.396(4)$ \\
\hline $\mathrm{C} 2-\mathrm{H} 2 \mathrm{~A}$ & $0.93(3)$ & $\mathrm{C} 26-\mathrm{C} 27$ & $1.384(5)$ \\
\hline $\mathrm{C} 3-\mathrm{C} 4$ & $1.421(5)$ & $\mathrm{C} 26-\mathrm{C} 31$ & $1.499(5)$ \\
\hline $\mathrm{C} 3-\mathrm{H} 3 \mathrm{~A}$ & $0.94(4)$ & $\mathrm{C} 27-\mathrm{C} 28$ & $1.371(5)$ \\
\hline $\mathrm{C} 4-\mathrm{C} 12$ & $1.465(5)$ & $\mathrm{C} 27-\mathrm{H} 27 \mathrm{~A}$ & 0.9500 \\
\hline $\mathrm{C} 4-\mathrm{H} 4 \mathrm{~A}$ & $0.91(3)$ & $\mathrm{C} 28-\mathrm{C} 29$ & $1.379(5)$ \\
\hline $\mathrm{C} 5-\mathrm{C} 6$ & $1.375(5)$ & $\mathrm{C} 28-\mathrm{H} 28 \mathrm{~A}$ & 0.9500 \\
\hline $\mathrm{C} 5-\mathrm{C} 13$ & $1.411(5)$ & $\mathrm{C} 29-\mathrm{C} 30$ & $1.386(5)$ \\
\hline $\mathrm{C} 5-\mathrm{H} 5 \mathrm{~A}$ & 0.9500 & $\mathrm{C} 29-\mathrm{H} 29 \mathrm{~A}$ & 0.9500 \\
\hline $\mathrm{C} 6-\mathrm{C} 7$ & $1.395(6)$ & $\mathrm{C} 30-\mathrm{C} 32$ & $1.496(5)$ \\
\hline C6-H6A & 0.9500 & $\mathrm{C} 31-\mathrm{H} 31 \mathrm{~A}$ & 0.9800 \\
\hline $\mathrm{C} 7-\mathrm{C} 8$ & $1.363(6)$ & $\mathrm{C} 31-\mathrm{H} 31 \mathrm{~B}$ & 0.9800 \\
\hline C7-H7A & 0.9500 & $\mathrm{C} 31-\mathrm{H} 31 \mathrm{C}$ & 0.9800 \\
\hline $\mathrm{C} 8-\mathrm{C} 14$ & $1.414(5)$ & $\mathrm{C} 32-\mathrm{H} 32 \mathrm{~A}$ & 0.9800 \\
\hline $\mathrm{C} 8-\mathrm{H} 8 \mathrm{~A}$ & 0.9500 & $\mathrm{C} 32-\mathrm{H} 32 \mathrm{~B}$ & 0.9800 \\
\hline $\mathrm{C} 9-\mathrm{C} 11$ & $1.367(5)$ & $\mathrm{C} 32-\mathrm{H} 32 \mathrm{C}$ & 0.9800 \\
\hline $\mathrm{C} 9-\mathrm{C} 14$ & $1.424(5)$ & $\mathrm{C} 33-\mathrm{N} 3$ & $1.181(4)$ \\
\hline C9- H9A & 0.9500 & $\mathrm{~N} 3-\mathrm{C} 34$ & $1.382(4)$ \\
\hline $\mathrm{C} 10-\mathrm{C} 12$ & $1.371(4)$ & $\mathrm{C} 34-\mathrm{C} 35$ & $1.393(5)$ \\
\hline $\mathrm{C} 10-\mathrm{C} 13$ & $1.413(5)$ & $\mathrm{C} 34-\mathrm{C} 39$ & $1.395(5)$ \\
\hline $\mathrm{C} 10-\mathrm{H} 10 \mathrm{~A}$ & 0.9500 & $\mathrm{C} 35-\mathrm{C} 36$ & $1.386(6)$ \\
\hline $\mathrm{C} 11-\mathrm{C} 12$ & $1.425(4)$ & $\mathrm{C} 35-\mathrm{C} 40$ & $1.494(6)$ \\
\hline $\mathrm{C} 13-\mathrm{C} 14$ & $1.425(5)$ & $\mathrm{C} 36-\mathrm{C} 37$ & $1.369(6)$ \\
\hline $\mathrm{C} 15-\mathrm{N} 1$ & $1.167(4)$ & $\mathrm{C} 36-\mathrm{H} 36 \mathrm{~A}$ & 0.9500 \\
\hline $\mathrm{N} 1-\mathrm{C} 16$ & $1.403(4)$ & $\mathrm{C} 37-\mathrm{C} 38$ & $1.393(6)$ \\
\hline $\mathrm{C} 16-\mathrm{C} 21$ & $1.392(4)$ & $\mathrm{C} 37-\mathrm{H} 37 \mathrm{~A}$ & 0.9500 \\
\hline $\mathrm{C} 16-\mathrm{C} 17$ & $1.398(5)$ & $\mathrm{C} 38-\mathrm{C} 39$ & $1.386(5)$ \\
\hline $\mathrm{C} 17-\mathrm{C} 18$ & $1.378(5)$ & $\mathrm{C} 38-\mathrm{H} 38 \mathrm{~A}$ & 0.9500 \\
\hline $\mathrm{C} 17-\mathrm{C} 22$ & $1.507(4)$ & $\mathrm{C} 39-\mathrm{C} 41$ & $1.505(5)$ \\
\hline $\mathrm{C} 18-\mathrm{C} 19$ & $1.384(5)$ & $\mathrm{C} 40-\mathrm{H} 40 \mathrm{~B}$ & 0.9800 \\
\hline $\mathrm{C} 18-\mathrm{H} 18 \mathrm{~A}$ & 0.9500 & $\mathrm{C} 40-\mathrm{H} 40 \mathrm{C}$ & 0.9800 \\
\hline $\mathrm{C} 19-\mathrm{C} 20$ & $1.367(5)$ & $\mathrm{C} 40-\mathrm{H} 40 \mathrm{D}$ & 0.9800 \\
\hline C19-H19A & 0.9500 & $\mathrm{C} 41-\mathrm{H} 41 \mathrm{~B}$ & 0.9800 \\
\hline $\mathrm{C} 20-\mathrm{C} 21$ & $1.393(4)$ & $\mathrm{C} 41-\mathrm{H} 41 \mathrm{C}$ & 0.9800 \\
\hline $\mathrm{C} 20-\mathrm{H} 20 \mathrm{~A}$ & 0.9500 & $\mathrm{C} 41-\mathrm{H} 41 \mathrm{D}$ & 0.9800 \\
\hline
\end{tabular}




\begin{tabular}{|c|c|c|c|}
\hline $\mathrm{C} 33-\mathrm{Fe} 1-\mathrm{C} 24$ & $90.40(14)$ & $\mathrm{C} 20-\mathrm{C} 19-\mathrm{C} 18$ & $120.3(3)$ \\
\hline $\mathrm{C} 33-\mathrm{Fe} 1-\mathrm{C} 15$ & $94.97(14)$ & $\mathrm{C} 20-\mathrm{C} 19-\mathrm{H} 19 \mathrm{~A}$ & 119.9 \\
\hline $\mathrm{C} 24-\mathrm{Fe} 1-\mathrm{C} 15$ & $100.38(14)$ & $\mathrm{C} 18-\mathrm{C} 19-\mathrm{H} 19 \mathrm{~A}$ & 119.9 \\
\hline $\mathrm{C} 33-\mathrm{Fe} 1-\mathrm{C} 3$ & $94.45(15)$ & $\mathrm{C} 19-\mathrm{C} 20-\mathrm{C} 21$ & $120.9(3)$ \\
\hline $\mathrm{C} 24-\mathrm{Fe} 1-\mathrm{C} 3$ & $119.62(14)$ & $\mathrm{C} 19-\mathrm{C} 20-\mathrm{H} 20 \mathrm{~A}$ & 119.5 \\
\hline $\mathrm{C} 15-\mathrm{Fe} 1-\mathrm{C} 3$ & $138.74(14)$ & $\mathrm{C} 21-\mathrm{C} 20-\mathrm{H} 20 \mathrm{~A}$ & 119.5 \\
\hline $\mathrm{C} 33-\mathrm{Fe} 1-\mathrm{C} 2$ & $128.22(15)$ & $\mathrm{C} 16-\mathrm{C} 21-\mathrm{C} 20$ & $117.4(3)$ \\
\hline $\mathrm{C} 24-\mathrm{Fe} 1-\mathrm{C} 2$ & $94.40(14)$ & $\mathrm{C} 16-\mathrm{C} 21-\mathrm{C} 23$ & $121.4(3)$ \\
\hline $\mathrm{C} 15-\mathrm{Fe} 1-\mathrm{C} 2$ & $134.22(15)$ & $\mathrm{C} 20-\mathrm{C} 21-\mathrm{C} 23$ & $121.2(3)$ \\
\hline $\mathrm{C} 3-\mathrm{Fe} 1-\mathrm{C} 2$ & $40.14(14)$ & $\mathrm{C} 17-\mathrm{C} 22-\mathrm{H} 22 \mathrm{~A}$ & 109.5 \\
\hline $\mathrm{C} 33-\mathrm{Fe} 1-\mathrm{C} 4$ & $89.44(14)$ & $\mathrm{C} 17-\mathrm{C} 22-\mathrm{H} 22 \mathrm{~B}$ & 109.5 \\
\hline $\mathrm{C} 24-\mathrm{Fe} 1-\mathrm{C} 4$ & $159.38(13)$ & $\mathrm{H} 22 \mathrm{~A}-\mathrm{C} 22-\mathrm{H} 22 \mathrm{~B}$ & 109.5 \\
\hline $\mathrm{C} 15-\mathrm{Fe} 1-\mathrm{C} 4$ & $100.17(13)$ & $\mathrm{C} 17-\mathrm{C} 22-\mathrm{H} 22 \mathrm{C}$ & 109.5 \\
\hline $\mathrm{C} 3-\mathrm{Fe} 1-\mathrm{C} 4$ & $39.90(13)$ & $\mathrm{H} 22 \mathrm{~A}-\mathrm{C} 22-\mathrm{H} 22 \mathrm{C}$ & 109.5 \\
\hline $\mathrm{C} 2-\mathrm{Fe} 1-\mathrm{C} 4$ & $69.82(14)$ & $\mathrm{H} 22 \mathrm{~B}-\mathrm{C} 22-\mathrm{H} 22 \mathrm{C}$ & 109.5 \\
\hline $\mathrm{C} 33-\mathrm{Fe} 1-\mathrm{C} 1$ & $162.98(14)$ & $\mathrm{C} 21-\mathrm{C} 23-\mathrm{H} 23 \mathrm{~A}$ & 109.5 \\
\hline $\mathrm{C} 24-\mathrm{Fe} 1-\mathrm{C} 1$ & $101.02(14)$ & $\mathrm{C} 21-\mathrm{C} 23-\mathrm{H} 23 \mathrm{~B}$ & 109.5 \\
\hline $\mathrm{C} 15-\mathrm{Fe} 1-\mathrm{C} 1$ & $95.33(14)$ & $\mathrm{H} 23 \mathrm{~A}-\mathrm{C} 23-\mathrm{H} 23 \mathrm{~B}$ & 109.5 \\
\hline $\mathrm{C} 3-\mathrm{Fe} 1-\mathrm{C} 1$ & $68.97(14)$ & $\mathrm{C} 21-\mathrm{C} 23-\mathrm{H} 23 \mathrm{C}$ & 109.5 \\
\hline $\mathrm{C} 2-\mathrm{Fe} 1-\mathrm{C} 1$ & 39.09 (14) & $\mathrm{H} 23 \mathrm{~A}-\mathrm{C} 23-\mathrm{H} 23 \mathrm{C}$ & 109.5 \\
\hline $\mathrm{C} 4-\mathrm{Fe} 1-\mathrm{C} 1$ & $75.42(14)$ & $\mathrm{H} 23 \mathrm{~B}-\mathrm{C} 23-\mathrm{H} 23 \mathrm{C}$ & 109.5 \\
\hline $\mathrm{C} 2-\mathrm{C} 1-\mathrm{C} 11$ & $120.1(3)$ & $\mathrm{N} 2-\mathrm{C} 24-\mathrm{Fe} 1$ & $174.7(3)$ \\
\hline $\mathrm{C} 2-\mathrm{C} 1-\mathrm{Fe} 1$ & $65.6(2)$ & $\mathrm{C} 24-\mathrm{N} 2-\mathrm{C} 25$ & $174.1(3)$ \\
\hline $\mathrm{C} 11-\mathrm{C} 1-\mathrm{Fe} 1$ & $105.2(2)$ & $\mathrm{C} 30-\mathrm{C} 25-\mathrm{C} 26$ & $122.9(3)$ \\
\hline $\mathrm{C} 2-\mathrm{C} 1-\mathrm{H} 1 \mathrm{~A}$ & $118(2)$ & $\mathrm{C} 30-\mathrm{C} 25-\mathrm{N} 2$ & $119.0(3)$ \\
\hline $\mathrm{C} 11-\mathrm{C} 1-\mathrm{H} 1 \mathrm{~A}$ & $116(2)$ & $\mathrm{C} 26-\mathrm{C} 25-\mathrm{N} 2$ & $118.1(3)$ \\
\hline $\mathrm{Fe} 1-\mathrm{C} 1-\mathrm{H} 1 \mathrm{~A}$ & $122(2)$ & $\mathrm{C} 27-\mathrm{C} 26-\mathrm{C} 25$ & $117.4(3)$ \\
\hline $\mathrm{C} 3-\mathrm{C} 2-\mathrm{C} 1$ & $115.8(3)$ & $\mathrm{C} 27-\mathrm{C} 26-\mathrm{C} 31$ & $122.4(3)$ \\
\hline $\mathrm{C} 3-\mathrm{C} 2-\mathrm{Fe} 1$ & $69.5(2)$ & $\mathrm{C} 25-\mathrm{C} 26-\mathrm{C} 31$ & $120.3(3)$ \\
\hline $\mathrm{C} 1-\mathrm{C} 2-\mathrm{Fe} 1$ & $75.3(2)$ & $\mathrm{C} 28-\mathrm{C} 27-\mathrm{C} 26$ & $121.3(3)$ \\
\hline $\mathrm{C} 3-\mathrm{C} 2-\mathrm{H} 2 \mathrm{~A}$ & $120(2)$ & $\mathrm{C} 28-\mathrm{C} 27-\mathrm{H} 27 \mathrm{~A}$ & 119.3 \\
\hline $\mathrm{C} 1-\mathrm{C} 2-\mathrm{H} 2 \mathrm{~A}$ & $124(2)$ & $\mathrm{C} 26-\mathrm{C} 27-\mathrm{H} 27 \mathrm{~A}$ & 119.3 \\
\hline $\mathrm{Fe} 1-\mathrm{C} 2-\mathrm{H} 2 \mathrm{~A}$ & $119(2)$ & $\mathrm{C} 27-\mathrm{C} 28-\mathrm{C} 29$ & $120.0(3)$ \\
\hline $\mathrm{C} 2-\mathrm{C} 3-\mathrm{C} 4$ & $115.6(3)$ & $\mathrm{C} 27-\mathrm{C} 28-\mathrm{H} 28 \mathrm{~A}$ & 120.0 \\
\hline $\mathrm{C} 2-\mathrm{C} 3-\mathrm{Fe} 1$ & $70.33(19)$ & $\mathrm{C} 29-\mathrm{C} 28-\mathrm{H} 28 \mathrm{~A}$ & 120.0 \\
\hline $\mathrm{C} 4-\mathrm{C} 3-\mathrm{Fe} 1$ & $73.50(19)$ & $\mathrm{C} 28-\mathrm{C} 29-\mathrm{C} 30$ & $121.5(3)$ \\
\hline $\mathrm{C} 2-\mathrm{C} 3-\mathrm{H} 3 \mathrm{~A}$ & $123(2)$ & $\mathrm{C} 28-\mathrm{C} 29-\mathrm{H} 29 \mathrm{~A}$ & 119.3 \\
\hline $\mathrm{C} 4-\mathrm{C} 3-\mathrm{H} 3 \mathrm{~A}$ & $121(2)$ & $\mathrm{C} 30-\mathrm{C} 29-\mathrm{H} 29 \mathrm{~A}$ & 119.3 \\
\hline $\mathrm{Fe} 1-\mathrm{C} 3-\mathrm{H} 3 \mathrm{~A}$ & $125(2)$ & $\mathrm{C} 29-\mathrm{C} 30-\mathrm{C} 25$ & $117.0(3)$ \\
\hline $\mathrm{C} 3-\mathrm{C} 4-\mathrm{C} 12$ & $119.8(3)$ & $\mathrm{C} 29-\mathrm{C} 30-\mathrm{C} 32$ & $121.7(3)$ \\
\hline $\mathrm{C} 3-\mathrm{C} 4-\mathrm{Fe} 1$ & $66.60(18)$ & $\mathrm{C} 25-\mathrm{C} 30-\mathrm{C} 32$ & $121.3(3)$ \\
\hline $\mathrm{C} 12-\mathrm{C} 4-\mathrm{Fe} 1$ & $106.0(2)$ & $\mathrm{C} 26-\mathrm{C} 31-\mathrm{H} 31 \mathrm{~A}$ & 109.5 \\
\hline $\mathrm{C} 3-\mathrm{C} 4-\mathrm{H} 4 \mathrm{~A}$ & $116(2)$ & $\mathrm{C} 26-\mathrm{C} 31-\mathrm{H} 31 \mathrm{~B}$ & 109.5 \\
\hline $\mathrm{C} 12-\mathrm{C} 4-\mathrm{H} 4 \mathrm{~A}$ & $118(2)$ & $\mathrm{H} 31 \mathrm{~A}-\mathrm{C} 31-\mathrm{H} 31 \mathrm{~B}$ & 109.5 \\
\hline $\mathrm{Fe} 1-\mathrm{C} 4-\mathrm{H} 4 \mathrm{~A}$ & $120(2)$ & $\mathrm{C} 26-\mathrm{C} 31-\mathrm{H} 31 \mathrm{C}$ & 109.5 \\
\hline $\mathrm{C} 6-\mathrm{C} 5-\mathrm{C} 13$ & $121.5(4)$ & $\mathrm{H} 31 \mathrm{~A}-\mathrm{C} 31-\mathrm{H} 31 \mathrm{C}$ & 109.5 \\
\hline $\mathrm{C} 6-\mathrm{C} 5-\mathrm{H} 5 \mathrm{~A}$ & 119.2 & $\mathrm{H} 31 \mathrm{~B}-\mathrm{C} 31-\mathrm{H} 31 \mathrm{C}$ & 109.5 \\
\hline $\mathrm{C} 13-\mathrm{C} 5-\mathrm{H} 5 \mathrm{~A}$ & 119.2 & $\mathrm{C} 30-\mathrm{C} 32-\mathrm{H} 32 \mathrm{~A}$ & 109.5 \\
\hline
\end{tabular}




\begin{tabular}{|c|c|}
\hline $\mathrm{C} 5-\mathrm{C} 6-\mathrm{C} 7$ & $119.9(4)$ \\
\hline $\mathrm{C} 5-\mathrm{C} 6-\mathrm{H} 6 \mathrm{~A}$ & 120.0 \\
\hline $\mathrm{C} 7-\mathrm{C} 6-\mathrm{H} 6 \mathrm{~A}$ & 120.0 \\
\hline $\mathrm{C} 8-\mathrm{C} 7-\mathrm{C} 6$ & $120.1(4)$ \\
\hline $\mathrm{C} 8-\mathrm{C} 7-\mathrm{H} 7 \mathrm{~A}$ & 119.9 \\
\hline $\mathrm{C} 6-\mathrm{C} 7-\mathrm{H} 7 \mathrm{~A}$ & 119.9 \\
\hline $\mathrm{C} 7-\mathrm{C} 8-\mathrm{C} 14$ & $121.8(4)$ \\
\hline $\mathrm{C} 7-\mathrm{C} 8-\mathrm{H} 8 \mathrm{~A}$ & 119.1 \\
\hline $\mathrm{C} 14-\mathrm{C} 8-\mathrm{H} 8 \mathrm{~A}$ & 119.1 \\
\hline $\mathrm{C} 11-\mathrm{C} 9-\mathrm{C} 14$ & $121.3(3)$ \\
\hline $\mathrm{C} 11-\mathrm{C} 9-\mathrm{H} 9 \mathrm{~A}$ & 119.3 \\
\hline $\mathrm{C} 14-\mathrm{C} 9-\mathrm{H} 9 \mathrm{~A}$ & 119.3 \\
\hline $\mathrm{C} 12-\mathrm{C} 10-\mathrm{C} 13$ & $121.0(3)$ \\
\hline $\mathrm{C} 12-\mathrm{C} 10-\mathrm{H} 10 \mathrm{~A}$ & 119.5 \\
\hline $\mathrm{C} 13-\mathrm{C} 10-\mathrm{H} 10 \mathrm{~A}$ & 119.5 \\
\hline $\mathrm{C} 9-\mathrm{C} 11-\mathrm{C} 12$ & $119.6(3)$ \\
\hline $\mathrm{C} 9-\mathrm{C} 11-\mathrm{C} 1$ & $126.6(3)$ \\
\hline $\mathrm{C} 12-\mathrm{C} 11-\mathrm{C} 1$ & $113.8(3)$ \\
\hline $\mathrm{C} 10-\mathrm{C} 12-\mathrm{C} 11$ & $120.3(3)$ \\
\hline $\mathrm{C} 10-\mathrm{C} 12-\mathrm{C} 4$ & $125.0(3)$ \\
\hline $\mathrm{C} 11-\mathrm{C} 12-\mathrm{C} 4$ & $114.6(3)$ \\
\hline $\mathrm{C} 5-\mathrm{C} 13-\mathrm{C} 10$ & $122.5(3)$ \\
\hline $\mathrm{C} 5-\mathrm{C} 13-\mathrm{C} 14$ & $118.4(3)$ \\
\hline $\mathrm{C} 10-\mathrm{C} 13-\mathrm{C} 14$ & $119.1(3)$ \\
\hline $\mathrm{C} 8-\mathrm{C} 14-\mathrm{C} 9$ & $123.2(4)$ \\
\hline $\mathrm{C} 8-\mathrm{C} 14-\mathrm{C} 13$ & $118.2(4)$ \\
\hline $\mathrm{C} 9-\mathrm{C} 14-\mathrm{C} 13$ & $118.6(3)$ \\
\hline $\mathrm{N} 1-\mathrm{C} 15-\mathrm{Fe} 1$ & $178.8(3)$ \\
\hline $\mathrm{C} 15-\mathrm{N} 1-\mathrm{C} 16$ & $177.5(4)$ \\
\hline $\mathrm{C} 21-\mathrm{C} 16-\mathrm{C} 17$ & $122.9(3)$ \\
\hline $\mathrm{C} 21-\mathrm{C} 16-\mathrm{N} 1$ & $118.3(3)$ \\
\hline $\mathrm{C} 17-\mathrm{C} 16-\mathrm{N} 1$ & $118.9(3)$ \\
\hline $\mathrm{C} 18-\mathrm{C} 17-\mathrm{C} 16$ & $117.1(3)$ \\
\hline $\mathrm{C} 18-\mathrm{C} 17-\mathrm{C} 22$ & $122.1(3)$ \\
\hline $\mathrm{C} 16-\mathrm{C} 17-\mathrm{C} 22$ & $120.8(3)$ \\
\hline $\mathrm{C} 17-\mathrm{C} 18-\mathrm{C} 19$ & $121.5(3)$ \\
\hline $\mathrm{C} 17-\mathrm{C} 18-\mathrm{H} 18 \mathrm{~A}$ & 119.3 \\
\hline $\mathrm{C} 19-\mathrm{C} 18-\mathrm{H} 18 \mathrm{~A}$ & 119.3 \\
\hline $\mathrm{C} 11-\mathrm{C} 1-\mathrm{C} 2-\mathrm{C} 3$ & $-35.8(5)$ \\
\hline $\mathrm{Fe} 1-\mathrm{C} 1-\mathrm{C} 2-\mathrm{C} 3$ & $58.2(3)$ \\
\hline $\mathrm{C} 11-\mathrm{C} 1-\mathrm{C} 2-\mathrm{Fe} 1$ & $-93.9(3)$ \\
\hline $\mathrm{C} 1-\mathrm{C} 2-\mathrm{C} 3-\mathrm{C} 4$ & $-1.7(4)$ \\
\hline $\mathrm{Fe} 1-\mathrm{C} 2-\mathrm{C} 3-\mathrm{C} 4$ & $59.6(3)$ \\
\hline $\mathrm{C} 1-\mathrm{C} 2-\mathrm{C} 3-\mathrm{Fe} 1$ & $-61.3(3)$ \\
\hline $\mathrm{C} 2-\mathrm{C} 3-\mathrm{C} 4-\mathrm{C} 12$ & $37.7(4)$ \\
\hline $\mathrm{Fe} 1-\mathrm{C} 3-\mathrm{C} 4-\mathrm{C} 12$ & $95.6(3)$ \\
\hline $\mathrm{C} 2-\mathrm{C} 3-\mathrm{C} 4-\mathrm{Fe} 1$ & $-57.9(3)$ \\
\hline
\end{tabular}

$\begin{array}{ll}\mathrm{C} 30-\mathrm{C} 32-\mathrm{H} 32 \mathrm{~B} & 109.5 \\ \mathrm{H} 32 \mathrm{~A}-\mathrm{C} 32-\mathrm{H} 32 \mathrm{~B} & 109.5 \\ \mathrm{C} 30-\mathrm{C} 32-\mathrm{H} 32 \mathrm{C} & 109.5 \\ \mathrm{H} 32 \mathrm{~A}-\mathrm{C} 32-\mathrm{H} 32 \mathrm{C} & 109.5 \\ \mathrm{H} 32 \mathrm{~B}-\mathrm{C} 32-\mathrm{H} 32 \mathrm{C} & 109.5 \\ \mathrm{~N} 3-\mathrm{C} 33-\mathrm{Fe} 1 & 174.9(3) \\ \mathrm{C} 33-\mathrm{N} 3-\mathrm{C} 34 & 166.5(3) \\ \mathrm{N} 3-\mathrm{C} 34-\mathrm{C} 35 & 118.1(3) \\ \mathrm{N} 3-\mathrm{C} 34-\mathrm{C} 39 & 118.9(3) \\ \mathrm{C} 35-\mathrm{C} 34-\mathrm{C} 39 & 122.9(3) \\ \mathrm{C} 36-\mathrm{C} 35-\mathrm{C} 34 & 117.2(4) \\ \mathrm{C} 36-\mathrm{C} 35-\mathrm{C} 40 & 122.7(4) \\ \mathrm{C} 34-\mathrm{C} 35-\mathrm{C} 40 & 120.1(4) \\ \mathrm{C} 37-\mathrm{C} 36-\mathrm{C} 35 & 121.4(4) \\ \mathrm{C} 37-\mathrm{C} 36-\mathrm{H} 36 \mathrm{~A} & 119.3\end{array}$

$\mathrm{C} 37-\mathrm{C} 36-\mathrm{H} 36 \mathrm{~A}$

$\mathrm{C} 35-\mathrm{C} 36-\mathrm{H} 36 \mathrm{~A} \quad 119.3$

$\mathrm{C} 36-\mathrm{C} 37-\mathrm{C} 38 \quad 120.4$ (4)

$\mathrm{C} 36-\mathrm{C} 37-\mathrm{H} 37 \mathrm{~A} \quad 119.8$

C38-C37-H37A $\quad 119.8$

$\mathrm{C} 39-\mathrm{C} 38-\mathrm{C} 37 \quad 120.4(4)$

$\mathrm{C} 39-\mathrm{C} 38-\mathrm{H} 38 \mathrm{~A} \quad 119.8$

$\mathrm{C} 37-\mathrm{C} 38-\mathrm{H} 38 \mathrm{~A} \quad 119.8$

$\mathrm{C} 38-\mathrm{C} 39-\mathrm{C} 34 \quad 117.5$ (3)

$\mathrm{C} 38-\mathrm{C} 39-\mathrm{C} 41 \quad 122.2(4)$

$\mathrm{C} 34-\mathrm{C} 39-\mathrm{C} 41 \quad 120.3$ (3)

$\mathrm{C} 35-\mathrm{C} 40-\mathrm{H} 40 \mathrm{~B} \quad 109.5$

$\mathrm{C} 35-\mathrm{C} 40-\mathrm{H} 40 \mathrm{C} \quad 109.5$

$\mathrm{H} 40 \mathrm{~B}-\mathrm{C} 40-\mathrm{H} 40 \mathrm{C} \quad 109.5$

$\mathrm{C} 35-\mathrm{C} 40-\mathrm{H} 40 \mathrm{D} \quad 109.5$

$\mathrm{H} 40 \mathrm{~B}-\mathrm{C} 40-\mathrm{H} 40 \mathrm{D} \quad 109.5$

$\mathrm{H} 40 \mathrm{C}-\mathrm{C} 40-\mathrm{H} 40 \mathrm{D} \quad 109.5$

$\mathrm{C} 39-\mathrm{C} 41-\mathrm{H} 41 \mathrm{~B} \quad 109.5$

$\mathrm{C} 39-\mathrm{C} 41-\mathrm{H} 41 \mathrm{C} \quad 109.5$

$\mathrm{H} 41 \mathrm{~B}-\mathrm{C} 41-\mathrm{H} 41 \mathrm{C} \quad 109.5$

C39-C41-H41D $\quad 109.5$

$\mathrm{H} 41 \mathrm{~B}-\mathrm{C} 41-\mathrm{H} 41 \mathrm{D} \quad 109.5$

H41C-C41-H41D $\quad 109.5$

$\begin{array}{ll}\mathrm{C} 21-\mathrm{C} 16-\mathrm{C} 17-\mathrm{C} 22 & -180.0(3) \\ \mathrm{N} 1-\mathrm{C} 16-\mathrm{C} 17-\mathrm{C} 22 & -0.4(5) \\ \mathrm{C} 16-\mathrm{C} 17-\mathrm{C} 18-\mathrm{C} 19 & 0.0(5) \\ \mathrm{C} 22-\mathrm{C} 17-\mathrm{C} 18-\mathrm{C} 19 & -179.2(3) \\ \mathrm{C} 17-\mathrm{C} 18-\mathrm{C} 19-\mathrm{C} 20 & -0.3(5) \\ \mathrm{C} 18-\mathrm{C} 19-\mathrm{C} 20-\mathrm{C} 21 & -0.2(5) \\ \mathrm{C} 17-\mathrm{C} 16-\mathrm{C} 21-\mathrm{C} 20 & -1.3(5) \\ \mathrm{N} 1-\mathrm{C} 16-\mathrm{C} 21-\mathrm{C} 20 & 179.1(3) \\ \mathrm{C} 17-\mathrm{C} 16-\mathrm{C} 21-\mathrm{C} 23 & 179.2(3)\end{array}$




\begin{tabular}{|c|c|c|c|}
\hline $\mathrm{C} 13-\mathrm{C} 5-\mathrm{C} 6-\mathrm{C} 7$ & $-0.2(5)$ & $\mathrm{N} 1-\mathrm{C} 16-\mathrm{C} 21-\mathrm{C} 23$ & $-0.4(5)$ \\
\hline $\mathrm{C} 5-\mathrm{C} 6-\mathrm{C} 7-\mathrm{C} 8$ & $-0.3(5)$ & $\mathrm{C} 19-\mathrm{C} 20-\mathrm{C} 21-\mathrm{C} 16$ & $0.9(5)$ \\
\hline $\mathrm{C} 6-\mathrm{C} 7-\mathrm{C} 8-\mathrm{C} 14$ & $-0.2(5)$ & $\mathrm{C} 19-\mathrm{C} 20-\mathrm{C} 21-\mathrm{C} 23$ & $-179.5(3)$ \\
\hline $\mathrm{C} 14-\mathrm{C} 9-\mathrm{C} 11-\mathrm{C} 12$ & $4.3(5)$ & $\mathrm{C} 30-\mathrm{C} 25-\mathrm{C} 26-\mathrm{C} 27$ & $-0.2(5)$ \\
\hline $\mathrm{C} 14-\mathrm{C} 9-\mathrm{C} 11-\mathrm{C} 1$ & $-174.6(3)$ & $\mathrm{N} 2-\mathrm{C} 25-\mathrm{C} 26-\mathrm{C} 27$ & $178.6(3)$ \\
\hline $\mathrm{C} 2-\mathrm{C} 1-\mathrm{C} 11-\mathrm{C} 9$ & $-144.3(4)$ & $\mathrm{C} 30-\mathrm{C} 25-\mathrm{C} 26-\mathrm{C} 31$ & $178.4(3)$ \\
\hline $\mathrm{Fe} 1-\mathrm{C} 1-\mathrm{C} 11-\mathrm{C} 9$ & $145.5(3)$ & $\mathrm{N} 2-\mathrm{C} 25-\mathrm{C} 26-\mathrm{C} 31$ & $-2.8(5)$ \\
\hline $\mathrm{C} 2-\mathrm{C} 1-\mathrm{C} 11-\mathrm{C} 12$ & $36.8(4)$ & $\mathrm{C} 25-\mathrm{C} 26-\mathrm{C} 27-\mathrm{C} 28$ & $0.6(5)$ \\
\hline $\mathrm{Fe} 1-\mathrm{C} 1-\mathrm{C} 11-\mathrm{C} 12$ & $-33.4(3)$ & $\mathrm{C} 31-\mathrm{C} 26-\mathrm{C} 27-\mathrm{C} 28$ & $-177.9(4)$ \\
\hline $\mathrm{C} 13-\mathrm{C} 10-\mathrm{C} 12-\mathrm{C} 11$ & $0.7(4)$ & $\mathrm{C} 26-\mathrm{C} 27-\mathrm{C} 28-\mathrm{C} 29$ & $-0.6(6)$ \\
\hline $\mathrm{C} 13-\mathrm{C} 10-\mathrm{C} 12-\mathrm{C} 4$ & $176.3(3)$ & $\mathrm{C} 27-\mathrm{C} 28-\mathrm{C} 29-\mathrm{C} 30$ & $0.2(6)$ \\
\hline $\mathrm{C} 9-\mathrm{C} 11-\mathrm{C} 12-\mathrm{C} 10$ & $-4.2(4)$ & $\mathrm{C} 28-\mathrm{C} 29-\mathrm{C} 30-\mathrm{C} 25$ & $0.1(5)$ \\
\hline $\mathrm{C} 1-\mathrm{C} 11-\mathrm{C} 12-\mathrm{C} 10$ & $174.8(3)$ & $\mathrm{C} 28-\mathrm{C} 29-\mathrm{C} 30-\mathrm{C} 32$ & $179.8(3)$ \\
\hline $\mathrm{C} 9-\mathrm{C} 11-\mathrm{C} 12-\mathrm{C} 4$ & $179.8(3)$ & $\mathrm{C} 26-\mathrm{C} 25-\mathrm{C} 30-\mathrm{C} 29$ & $-0.2(5)$ \\
\hline $\mathrm{C} 1-\mathrm{C} 11-\mathrm{C} 12-\mathrm{C} 4$ & $-1.2(4)$ & $\mathrm{N} 2-\mathrm{C} 25-\mathrm{C} 30-\mathrm{C} 29$ & $-179.0(3)$ \\
\hline $\mathrm{C} 3-\mathrm{C} 4-\mathrm{C} 12-\mathrm{C} 10$ & $148.6(3)$ & $\mathrm{C} 26-\mathrm{C} 25-\mathrm{C} 30-\mathrm{C} 32$ & $-179.8(3)$ \\
\hline $\mathrm{Fe} 1-\mathrm{C} 4-\mathrm{C} 12-\mathrm{C} 10$ & $-139.5(3)$ & $\mathrm{N} 2-\mathrm{C} 25-\mathrm{C} 30-\mathrm{C} 32$ & $1.4(5)$ \\
\hline $\mathrm{C} 3-\mathrm{C} 4-\mathrm{C} 12-\mathrm{C} 11$ & $-35.6(4)$ & $\mathrm{C} 33-\mathrm{N} 3-\mathrm{C} 34-\mathrm{C} 35$ & $2.0(15)$ \\
\hline $\mathrm{Fe} 1-\mathrm{C} 4-\mathrm{C} 12-\mathrm{C} 11$ & $36.2(3)$ & $\mathrm{C} 33-\mathrm{N} 3-\mathrm{C} 34-\mathrm{C} 39$ & $-177.6(13)$ \\
\hline $\mathrm{C} 6-\mathrm{C} 5-\mathrm{C} 13-\mathrm{C} 10$ & $179.4(3)$ & $\mathrm{N} 3-\mathrm{C} 34-\mathrm{C} 35-\mathrm{C} 36$ & $-176.0(3)$ \\
\hline $\mathrm{C} 6-\mathrm{C} 5-\mathrm{C} 13-\mathrm{C} 14$ & $1.2(5)$ & $\mathrm{C} 39-\mathrm{C} 34-\mathrm{C} 35-\mathrm{C} 36$ & $3.6(5)$ \\
\hline $\mathrm{C} 12-\mathrm{C} 10-\mathrm{C} 13-\mathrm{C} 5$ & $-175.7(3)$ & $\mathrm{N} 3-\mathrm{C} 34-\mathrm{C} 35-\mathrm{C} 40$ & $4.2(5)$ \\
\hline $\mathrm{C} 12-\mathrm{C} 10-\mathrm{C} 13-\mathrm{C} 14$ & $2.6(4)$ & $\mathrm{C} 39-\mathrm{C} 34-\mathrm{C} 35-\mathrm{C} 40$ & $-176.2(3)$ \\
\hline $\mathrm{C} 7-\mathrm{C} 8-\mathrm{C} 14-\mathrm{C} 9$ & $-176.2(3)$ & $\mathrm{C} 34-\mathrm{C} 35-\mathrm{C} 36-\mathrm{C} 37$ & $-0.1(5)$ \\
\hline $\mathrm{C} 7-\mathrm{C} 8-\mathrm{C} 14-\mathrm{C} 13$ & $1.2(5)$ & $\mathrm{C} 40-\mathrm{C} 35-\mathrm{C} 36-\mathrm{C} 37$ & $179.7(4)$ \\
\hline $\mathrm{C} 11-\mathrm{C} 9-\mathrm{C} 14-\mathrm{C} 8$ & $176.3(3)$ & $\mathrm{C} 35-\mathrm{C} 36-\mathrm{C} 37-\mathrm{C} 38$ & $-2.2(6)$ \\
\hline $\mathrm{C} 11-\mathrm{C} 9-\mathrm{C} 14-\mathrm{C} 13$ & $-1.0(5)$ & $\mathrm{C} 36-\mathrm{C} 37-\mathrm{C} 38-\mathrm{C} 39$ & $1.1(6)$ \\
\hline $\mathrm{C} 5-\mathrm{C} 13-\mathrm{C} 14-\mathrm{C} 8$ & $-1.6(4)$ & $\mathrm{C} 37-\mathrm{C} 38-\mathrm{C} 39-\mathrm{C} 34$ & $2.2(5)$ \\
\hline $\mathrm{C} 10-\mathrm{C} 13-\mathrm{C} 14-\mathrm{C} 8$ & $-179.9(3)$ & $\mathrm{C} 37-\mathrm{C} 38-\mathrm{C} 39-\mathrm{C} 41$ & $-176.7(3)$ \\
\hline $\mathrm{C} 5-\mathrm{C} 13-\mathrm{C} 14-\mathrm{C} 9$ & $175.8(3)$ & $\mathrm{N} 3-\mathrm{C} 34-\mathrm{C} 39-\mathrm{C} 38$ & $175.0(3)$ \\
\hline $\mathrm{C} 10-\mathrm{C} 13-\mathrm{C} 14-\mathrm{C} 9$ & $-2.4(4)$ & $\mathrm{C} 35-\mathrm{C} 34-\mathrm{C} 39-\mathrm{C} 38$ & $-4.6(5)$ \\
\hline $\mathrm{C} 21-\mathrm{C} 16-\mathrm{C} 17-\mathrm{C} 18$ & $0.8(5)$ & $\mathrm{N} 3-\mathrm{C} 34-\mathrm{C} 39-\mathrm{C} 41$ & $-6.2(5)$ \\
\hline $\mathrm{N} 1-\mathrm{C} 16-\mathrm{C} 17-\mathrm{C} 18$ & $-179.6(3)$ & $\mathrm{C} 35-\mathrm{C} 34-\mathrm{C} 39-\mathrm{C} 41$ & $174.2(3)$ \\
\hline
\end{tabular}

\{5,6-Bis(2,6-dimethylanilino)-3-(2,6-dimethylphenyl)-1,2,7-tris[(2,6-dimethylphenyl)imino]-3-azoniahept-3ene-1,4,7-triido\}tris(2,6-dimethylphenyl isocyanide)iron tetrahydrofuran disolvate (2)

\section{Crystal data}

$\left[\mathrm{Fe}\left(\mathrm{C}_{54} \mathrm{H}_{56} \mathrm{~N}_{6}\right)\left(\mathrm{C}_{9} \mathrm{H}_{9} \mathrm{~N}\right)_{3}\right] \cdot 2 \mathrm{C}_{4} \mathrm{H}_{8} \mathrm{O}$

$M_{r}=1382.62$

Triclinic, $P \overline{1}$

$a=13.8912(10) \AA$

$b=15.4941(11) \AA$

$c=19.7902(14) \AA$

$\alpha=85.342(3)^{\circ}$

$\beta=74.001(3)^{\circ}$

$\gamma=70.884(3)^{\circ}$

$V=3868.5(5) \AA^{3}$
$Z=2$

$F(000)=1476$

$D_{\mathrm{x}}=1.187 \mathrm{Mg} \mathrm{m}^{-3}$

Mo $K \alpha$ radiation, $\lambda=0.71073 \AA$

Cell parameters from 3451 reflections

$\theta=2.3-22.7^{\circ}$

$\mu=0.25 \mathrm{~mm}^{-1}$

$T=293 \mathrm{~K}$

Block, dark red

$0.34 \times 0.30 \times 0.24 \mathrm{~mm}$ 


\section{Data collection}

Bruker SMART CCD platform diffractometer

Radiation source: fine-focus sealed tube $\omega$ scans

Absorption correction: multi-scan

(SADABS; Krause et al., 2015)

$T_{\min }=0.925, T_{\max }=1.000$

31905 measured reflections

\section{Refinement}

Refinement on $F^{2}$

Least-squares matrix: full

$R\left[F^{2}>2 \sigma\left(F^{2}\right)\right]=0.051$

$w R\left(F^{2}\right)=0.136$

$S=1.02$

13640 reflections

1192 parameters

382 restraints

Primary atom site location: structure-invariant direct methods
13640 independent reflections

9367 reflections with $I>2 \sigma(I)$

$R_{\text {int }}=0.040$

$\theta_{\max }=25.1^{\circ}, \theta_{\min }=1.6^{\circ}$

$h=-16 \rightarrow 16$

$k=-18 \rightarrow 18$

$l=-23 \rightarrow 23$

Secondary atom site location: difference Fourier map

Hydrogen site location: mixed

$\mathrm{H}$ atoms treated by a mixture of independent and constrained refinement

$w=1 /\left[\sigma^{2}\left(F_{\mathrm{o}}^{2}\right)+(0.060 P)^{2}+0.9501 P\right]$ where $P=\left(F_{\mathrm{o}}^{2}+2 F_{\mathrm{c}}^{2}\right) / 3$

$(\Delta / \sigma)_{\max }<0.001$

$\Delta \rho_{\max }=0.38 \mathrm{e} \AA^{-3}$

$\Delta \rho_{\min }=-0.18$ e $\AA^{-3}$

\section{Special details}

Geometry. All esds (except the esd in the dihedral angle between two 1.s. planes) are estimated using the full covariance matrix. The cell esds are taken into account individually in the estimation of esds in distances, angles and torsion angles; correlations between esds in cell parameters are only used when they are defined by crystal symmetry. An approximate (isotropic) treatment of cell esds is used for estimating esds involving l.s. planes.

Refinement. Two CNXyl groups were modeled as disordered over two positions each: N1/C2-C9, 0.52 (2):0.48 (2) and N8/C65-C72, 0.57 (2):0.43 (2). The two thf solvent molecules were modeled as disordered over two positions each: $\mathrm{O} 1 / \mathrm{C} 82-\mathrm{C} 85,0.55$ (2):0.45 (2) and O2/C86-C89, 0.69 (1):0.31 (1).

For the various pairs of components of disorder, analogous bond lengths and angles were restrained to be similar and anisotropic displacement parameters for proximal atoms were restrained to be similar. Bond lengths for the thf solvent molecules were restrained toward ideal values. Anisotropic displacement parameters for the thf solvent molecules were also restrained toward the expected motion relative to bond direction.

Fractional atomic coordinates and isotropic or equivalent isotropic displacement parameters $\left(\AA^{2}\right)$

\begin{tabular}{llllll}
\hline & $x$ & $y$ & $z$ & $U_{\text {iso }} * U_{\text {eq }}$ & Occ. $(<1)$ \\
\hline Fe1 & $0.35113(3)$ & $0.21957(2)$ & $0.30062(2)$ & $0.03572(11)$ & \\
C1 & $0.25165(18)$ & $0.18309(16)$ & $0.36939(12)$ & $0.0374(5)$ & $0.48(2)$ \\
N1 & $0.1973(17)$ & $0.163(2)$ & $0.4210(8)$ & $0.038(3)$ & $0.48(2)$ \\
C2 & $0.1256(12)$ & $0.1360(14)$ & $0.4769(8)$ & $0.040(3)$ & $0.48(2)$ \\
C3 & $0.1659(12)$ & $0.0698(12)$ & $0.5224(9)$ & $0.050(3)$ & $0.48(2)$ \\
C4 & $0.0935(14)$ & $0.0492(11)$ & $0.5792(8)$ & $0.084(4)$ & $0.48(2)$ \\
H4 & 0.117586 & 0.005216 & 0.610856 & $0.101^{*}$ & $0.48(2)$ \\
C5 & $-0.0125(14)$ & $0.0917(13)$ & $0.5900(10)$ & $0.114(5)$ & $0.48(2)$ \\
H5 & -0.059463 & 0.077798 & 0.629450 & $0.137 *$ & $0.48(2)$ \\
C6 & $-0.0499(12)$ & $0.1545(12)$ & $0.5433(9)$ & $0.088(4)$ & $0.48(2)$ \\
H6 & -0.122488 & 0.181145 & 0.550534 & $0.106 *$ & $0.48(2)$ \\
C7 & $0.0172(12)$ & $0.1794(11)$ & $0.4858(8)$ & $0.053(3)$ & $0.48(2)$ \\
C8 & $0.2805(13)$ & $0.017(2)$ & $0.5158(18)$ & $0.069(5)$ & $0.103 *$ \\
H8A & 0.286422 & -0.042250 & 0.535705 & $(2)$
\end{tabular}




\begin{tabular}{|c|c|c|c|c|c|}
\hline H8B & 0.309270 & 0.049390 & 0.540343 & $0.103^{*}$ & $0.48(2)$ \\
\hline $\mathrm{H} 8 \mathrm{C}$ & 0.318798 & 0.011109 & 0.467017 & $0.103 *$ & $0.48(2)$ \\
\hline C9 & $-0.035(2)$ & $0.2440(19)$ & $0.4353(16)$ & $0.089(6)$ & $0.48(2)$ \\
\hline H9A & -0.054436 & 0.210192 & 0.405471 & $0.133 *$ & $0.48(2)$ \\
\hline H9B & 0.012745 & 0.273556 & 0.407097 & $0.133^{*}$ & $0.48(2)$ \\
\hline $\mathrm{H} 9 \mathrm{C}$ & -0.097647 & 0.289155 & 0.461464 & $0.133^{*}$ & $0.48(2)$ \\
\hline $\mathrm{N} 1^{\prime}$ & $0.1885(16)$ & 0.1561 (19) & $0.4099(8)$ & $0.039(3)$ & $0.52(2)$ \\
\hline $\mathrm{C} 2^{\prime}$ & $0.1201(11)$ & $0.1246(12)$ & $0.4650(8)$ & $0.040(3)$ & $0.52(2)$ \\
\hline $\mathrm{C} 3^{\prime}$ & $0.1615(12)$ & $0.0563(12)$ & $0.5086(8)$ & $0.050(3)$ & $0.52(2)$ \\
\hline $\mathrm{C} 4^{\prime}$ & $0.0903(12)$ & $0.0241(10)$ & $0.5586(8)$ & $0.078(3)$ & $0.52(2)$ \\
\hline $\mathrm{H}^{\prime \prime}$ & 0.115114 & -0.021889 & 0.588672 & $0.093 *$ & $0.52(2)$ \\
\hline $\mathrm{C} 5^{\prime}$ & $-0.0159(11)$ & $0.0583(12)$ & $0.5648(8)$ & $0.099(4)$ & $0.52(2)$ \\
\hline H5' & -0.062225 & 0.034129 & 0.597690 & $0.119^{*}$ & $0.52(2)$ \\
\hline $\mathrm{C} 6^{\prime}$ & $-0.0538(11)$ & $0.1280(11)$ & $0.5227(8)$ & $0.082(4)$ & $0.52(2)$ \\
\hline H6 & -0.126460 & 0.152483 & 0.528949 & $0.098 *$ & $0.52(2)$ \\
\hline$C 7^{\prime}$ & $0.0115(11)$ & $0.1629(10)$ & $0.4718(7)$ & $0.052(3)$ & $0.52(2)$ \\
\hline $\mathrm{C} 8^{\prime}$ & $0.2783(13)$ & $0.024(2)$ & $0.5028(18)$ & $0.074(5)$ & $0.52(2)$ \\
\hline H8D & 0.313260 & 0.054203 & 0.463980 & $0.111^{*}$ & $0.52(2)$ \\
\hline H8E & 0.306287 & -0.040505 & 0.495216 & $0.111^{*}$ & $0.52(2)$ \\
\hline $\mathrm{H} 8 \mathrm{~F}$ & 0.289881 & 0.039270 & 0.545434 & $0.111^{*}$ & $0.52(2)$ \\
\hline $\mathrm{C} 9^{\prime}$ & $-0.024(2)$ & $0.2447(16)$ & $0.4271(14)$ & $0.081(5)$ & $0.52(2)$ \\
\hline H9D & 0.026936 & 0.238549 & 0.382300 & $0.121 *$ & $0.52(2)$ \\
\hline H9E & -0.031125 & 0.299351 & 0.450271 & $0.121^{*}$ & $0.52(2)$ \\
\hline H9F & -0.091234 & 0.248324 & 0.420245 & $0.121^{*}$ & $0.52(2)$ \\
\hline $\mathrm{C} 10$ & $0.34098(19)$ & $0.29901(17)$ & $0.36912(13)$ & $0.0430(6)$ & \\
\hline $\mathrm{N} 2$ & $0.32553(18)$ & $0.34287(15)$ & $0.41886(12)$ & $0.0532(6)$ & \\
\hline C11 & $0.3071(2)$ & 0.39771 (19) & $0.47604(16)$ & $0.0601(8)$ & \\
\hline C12 & 0.3699 & $0.4569(2)$ & $0.4668(2)$ & $0.0751(10)$ & \\
\hline C13 & $0.3503(4)$ & $0.5122(3)$ & 0.5228 & $0.1084(15)$ & \\
\hline H13 & 0.389612 & 0.551578 & 0.519289 & $0.130 *$ & \\
\hline C14 & $0.2763(4)$ & $0.5113(3)$ & $0.5825(3)$ & $0.1104(17)$ & \\
\hline H14 & 0.265382 & 0.550597 & 0.618980 & $0.133^{*}$ & \\
\hline $\mathrm{C} 15$ & 0.2149 & $0.4537(3)$ & $0.5921(2)$ & 0.1005 (14) & \\
\hline H15 & 0.164033 & 0.454590 & 0.634154 & $0.121 *$ & \\
\hline $\mathrm{C} 16$ & $0.2314(3)$ & $0.3935(2)$ & $0.53630(17)$ & $0.0723(9)$ & \\
\hline $\mathrm{C} 17$ & $0.4513(3)$ & $0.4572(3)$ & $0.4001(2)$ & $0.0970(12)$ & \\
\hline H17A & 0.419412 & 0.468432 & 0.361580 & $0.146^{*}$ & \\
\hline H17B & 0.505805 & 0.398944 & 0.393440 & $0.146^{*}$ & \\
\hline $\mathrm{H} 17 \mathrm{C}$ & 0.481311 & 0.504254 & 0.402151 & $0.146^{*}$ & \\
\hline $\mathrm{C} 18$ & 0.1678 & 0.3303 & $0.54419(18)$ & 0.0888 (11) & \\
\hline H18A & 0.110799 & 0.345926 & 0.586198 & $0.133^{*}$ & \\
\hline H18B & 0.212062 & 0.268604 & 0.547200 & $0.133^{*}$ & \\
\hline $\mathrm{H} 18 \mathrm{C}$ & 0.139776 & 0.335815 & 0.504237 & $0.133^{*}$ & \\
\hline C19 & $0.4540(2)$ & $0.25518(16)$ & $0.23412(13)$ & $0.0433(6)$ & \\
\hline N3 & $0.51689(18)$ & $0.27967(16)$ & $0.19195(12)$ & $0.0577(6)$ & \\
\hline $\mathrm{C} 20$ & $0.5842(2)$ & $0.3167(2)$ & $0.13985(18)$ & $0.0667(8)$ & \\
\hline $\mathrm{C} 21$ & $0.5967(3)$ & 0.2979 & $0.0692(2)$ & $0.0917(12)$ & \\
\hline $\mathrm{C} 22$ & $0.6598(5)$ & $0.3372(4)$ & $0.0190(3)$ & $0.141(2)$ & \\
\hline
\end{tabular}




\begin{tabular}{|c|c|c|c|c|}
\hline $\mathrm{H} 22$ & 0.669592 & 0.326608 & -0.028387 & $0.169 *$ \\
\hline $\mathrm{C} 23$ & $0.7074(5)$ & $0.3904(5)$ & $0.0376(4)$ & $0.158(2)$ \\
\hline $\mathrm{H} 23$ & 0.749511 & 0.415784 & 0.002305 & $0.190^{*}$ \\
\hline $\mathrm{C} 24$ & $0.6971(4)$ & $0.4091(4)$ & $0.1052(3)$ & $0.1276(18)$ \\
\hline $\mathrm{H} 24$ & 0.732090 & 0.445884 & 0.115935 & $0.153 *$ \\
\hline $\mathrm{C} 25$ & $0.6318(3)$ & $0.3716(3)$ & $0.1599(2)$ & $0.0947(12)$ \\
\hline $\mathrm{C} 26$ & $0.5409(4)$ & $0.2409(3)$ & $0.0490(2)$ & $0.1274(17)$ \\
\hline $\mathrm{H} 26 \mathrm{~A}$ & 0.468143 & 0.260308 & 0.075514 & $0.191 *$ \\
\hline $\mathrm{H} 26 \mathrm{~B}$ & 0.573494 & 0.177966 & 0.058805 & $0.191^{*}$ \\
\hline $\mathrm{H} 26 \mathrm{C}$ & 0.545128 & 0.247637 & -0.000267 & $0.191^{*}$ \\
\hline $\mathrm{C} 27$ & 0.6145 & $0.3914(3)$ & 0.2352 & $0.1335(18)$ \\
\hline $\mathrm{H} 27 \mathrm{~A}$ & 0.640446 & 0.440613 & 0.239048 & $0.200^{*}$ \\
\hline $\mathrm{H} 27 \mathrm{~B}$ & 0.651428 & 0.337972 & 0.256977 & $0.200 *$ \\
\hline $\mathrm{H} 27 \mathrm{C}$ & 0.540285 & 0.408553 & 0.258234 & $0.200^{*}$ \\
\hline $\mathrm{C} 28$ & 0.24879 (19) & $0.30335(17)$ & $0.25034(12)$ & $0.0406(6)$ \\
\hline N4 & $0.20694(17)$ & $0.38993(14)$ & $0.24343(12)$ & $0.0509(5)$ \\
\hline $\mathrm{C} 29$ & $0.2172(2)$ & $0.45872(17)$ & $0.28145(16)$ & $0.0539(7)$ \\
\hline $\mathrm{C} 30$ & $0.2816(2)$ & $0.51023(19)$ & $0.24651(18)$ & $0.0640(8)$ \\
\hline $\mathrm{C} 31$ & 0.2848 & $0.5822(2)$ & $0.2823(2)$ & $0.0820(10)$ \\
\hline H31 & 0.328483 & 0.616191 & 0.259955 & $0.098^{*}$ \\
\hline $\mathrm{C} 32$ & 0.2248 & $0.6042(2)$ & $0.3502(2)$ & $0.0910(12)$ \\
\hline H32 & 0.229517 & 0.651535 & 0.373849 & $0.109^{*}$ \\
\hline $\mathrm{C} 33$ & 0.1577 (3) & $0.5562(2)$ & 0.38299 (19) & $0.0775(10)$ \\
\hline H33 & 0.115221 & 0.572878 & 0.428293 & $0.093 *$ \\
\hline C34 & $0.1524(2)$ & 0.48277 (19) & $0.34933(17)$ & $0.0608(8)$ \\
\hline $\mathrm{C} 35$ & 0.3405 & $0.4929(2)$ & $0.17021(19)$ & $0.0876(11)$ \\
\hline $\mathrm{H} 35 \mathrm{~A}$ & 0.377563 & 0.428753 & 0.162594 & $0.131 *$ \\
\hline H35B & 0.390138 & 0.526179 & 0.157491 & $0.131^{*}$ \\
\hline $\mathrm{H} 35 \mathrm{C}$ & 0.291318 & 0.512636 & 0.141852 & $0.131 *$ \\
\hline $\mathrm{C} 36$ & $0.0763(3)$ & $0.4330(2)$ & $0.38418(18)$ & $0.0772(9)$ \\
\hline H36A & 0.114516 & 0.370046 & 0.389743 & $0.116^{*}$ \\
\hline H36B & 0.029164 & 0.437033 & 0.355702 & $0.116^{*}$ \\
\hline $\mathrm{H} 36 \mathrm{C}$ & 0.036362 & 0.460045 & 0.429484 & $0.116^{*}$ \\
\hline $\mathrm{C} 37$ & 0.21966 (19) & $0.25081(16)$ & $0.20101(12)$ & $0.0395(6)$ \\
\hline N5 & $0.15384(17)$ & $0.27524(14)$ & $0.16458(11)$ & $0.0496(5)$ \\
\hline $\mathrm{C} 38$ & $0.0871(2)$ & $0.36446(18)$ & $0.15718(15)$ & $0.0548(7)$ \\
\hline C39 & -0.0167 & $0.3937(2)$ & $0.19960(18)$ & $0.0687(9)$ \\
\hline $\mathrm{C} 40$ & $-0.0847(3)$ & 0.4763 & 0.1850 & $0.0979(12)$ \\
\hline $\mathrm{H} 40$ & -0.153475 & 0.496925 & 0.213467 & $0.118^{*}$ \\
\hline $\mathrm{C} 41$ & $-0.0532(4)$ & 0.5280 & 0.1299 & $0.1214(17)$ \\
\hline H41 & -0.099957 & 0.583743 & 0.121359 & $0.146^{*}$ \\
\hline $\mathrm{C} 42$ & $0.0480(4)$ & $0.4979(3)$ & $0.0867(2)$ & $0.1056(14)$ \\
\hline $\mathrm{H} 42$ & 0.068513 & 0.532855 & 0.048386 & $0.127 *$ \\
\hline $\mathrm{C} 43$ & $0.1197(3)$ & $0.4162(2)$ & $0.09944(17)$ & $0.0698(9)$ \\
\hline $\mathrm{C} 44$ & -0.0534 & $0.3352(3)$ & $0.2585(2)$ & $0.0938(11)$ \\
\hline $\mathrm{H} 44 \mathrm{~A}$ & -0.040509 & 0.275608 & 0.240580 & $0.141^{*}$ \\
\hline H44B & -0.127639 & 0.362572 & 0.279375 & $0.141^{*}$ \\
\hline $\mathrm{H} 44 \mathrm{C}$ & -0.015496 & 0.330053 & 0.293340 & $0.141 *$ \\
\hline
\end{tabular}




\begin{tabular}{|c|c|c|c|c|c|}
\hline $\mathrm{C} 45$ & $0.2297(3)$ & $0.3817(3)$ & 0.05277 (19) & $0.0975(12)$ & \\
\hline $\mathrm{H} 45 \mathrm{~A}$ & 0.244439 & 0.319767 & 0.038922 & $0.146^{*}$ & \\
\hline $\mathrm{H} 45 \mathrm{~B}$ & 0.279026 & 0.384155 & 0.077726 & $0.146^{*}$ & \\
\hline $\mathrm{H} 45 \mathrm{C}$ & 0.236145 & 0.419151 & 0.011719 & $0.146^{*}$ & \\
\hline $\mathrm{C} 46$ & $0.34745(17)$ & $0.12871(16)$ & $0.23919(11)$ & $0.0358(5)$ & \\
\hline N6 & $0.27881(15)$ & $0.15579(13)$ & $0.19868(10)$ & $0.0387(5)$ & \\
\hline $\mathrm{C} 47$ & $0.2492(2)$ & $0.09565(17)$ & $0.16096(14)$ & $0.0475(6)$ & \\
\hline $\mathrm{C} 48$ & $0.1703(2)$ & 0.05989 (19) & $0.19782(17)$ & $0.0618(8)$ & \\
\hline C49 & $0.1442(3)$ & 0.0007 (3) & $0.1624(3)$ & $0.0936(12)$ & \\
\hline $\mathrm{H} 49$ & 0.092437 & -0.024861 & 0.186113 & $0.112 *$ & \\
\hline $\mathrm{C} 50$ & $0.1938(4)$ & $-0.0203(3)$ & $0.0931(3)$ & $0.1080(15)$ & \\
\hline $\mathrm{H} 50$ & 0.175252 & -0.060106 & 0.070233 & $0.130^{*}$ & \\
\hline $\mathrm{C} 51$ & $0.2700(4)$ & $0.0162(2)$ & $0.0569(2)$ & $0.0883(12)$ & \\
\hline H51 & 0.302034 & 0.001541 & 0.009619 & $0.106^{*}$ & \\
\hline C52 & $0.3005(3)$ & $0.07522(19)$ & $0.08996(15)$ & $0.0597(8)$ & \\
\hline $\mathrm{C} 53$ & $0.1133(3)$ & $0.0839(2)$ & $0.27325(19)$ & $0.0793(10)$ & \\
\hline H53A & 0.090769 & 0.148977 & 0.279523 & $0.119 *$ & \\
\hline H53B & 0.159759 & 0.055074 & 0.302332 & $0.119^{*}$ & \\
\hline $\mathrm{H} 53 \mathrm{C}$ & 0.052766 & 0.063136 & 0.286284 & $0.119^{*}$ & \\
\hline C54 & 0.3860 & $0.1129(2)$ & $0.05188(16)$ & $0.0798(10)$ & \\
\hline H54A & 0.362848 & 0.177606 & 0.060169 & $0.120^{*}$ & \\
\hline H54B & 0.402603 & 0.101166 & 0.002415 & $0.120^{*}$ & \\
\hline $\mathrm{H} 54 \mathrm{C}$ & 0.447690 & 0.084315 & 0.068461 & $0.120^{*}$ & \\
\hline C55 & $0.40919(18)$ & $0.03657(16)$ & $0.24366(12)$ & $0.0376(5)$ & \\
\hline N7 & $0.42428(19)$ & $-0.03613(14)$ & $0.19870(11)$ & $0.0469(5)$ & \\
\hline $\mathrm{H} 7$ & $0.368(2)$ & $-0.0414(17)$ & $0.1943(13)$ & $0.047(8)^{*}$ & \\
\hline C56 & $0.5200(2)$ & $-0.10162(18)$ & $0.16579(13)$ & $0.0517(7)$ & \\
\hline C57 & 0.5144 & $-0.1858(2)$ & $0.14824(15)$ & $0.0666(9)$ & \\
\hline C58 & $0.6081(4)$ & $-0.2537(2)$ & $0.11867(18)$ & $0.0911(12)$ & \\
\hline $\mathrm{H} 58$ & 0.605816 & -0.309865 & 0.107167 & $0.109^{*}$ & \\
\hline C59 & $0.7037(4)$ & $-0.2398(3)$ & $0.1061(2)$ & $0.1055(16)$ & \\
\hline H59 & 0.765816 & -0.287274 & 0.088764 & $0.127^{*}$ & \\
\hline $\mathrm{C} 60$ & 0.7078 & $-0.1557(3)$ & $0.11908(17)$ & $0.0909(12)$ & \\
\hline H60 & 0.773083 & -0.146271 & 0.108416 & $0.109^{*}$ & \\
\hline C61 & $0.6169(2)$ & $-0.0840(2)$ & $0.14781(14)$ & $0.0623(8)$ & \\
\hline C62 & $0.4103(3)$ & $-0.2018(2)$ & $0.15989(18)$ & $0.0833(11)$ & \\
\hline H62A & 0.371305 & -0.189685 & 0.208266 & $0.125^{*}$ & \\
\hline H62B & 0.422128 & -0.264150 & 0.148473 & $0.125^{*}$ & \\
\hline $\mathrm{H} 62 \mathrm{C}$ & 0.370665 & -0.161861 & 0.130402 & $0.125^{*}$ & \\
\hline C63 & $0.6280(2)$ & $0.0076(2)$ & $0.15438(16)$ & $0.0756(9)$ & \\
\hline H63A & 0.572458 & 0.054107 & 0.139812 & $0.113^{*}$ & \\
\hline H63B & 0.695168 & 0.009104 & 0.125112 & $0.113 *$ & \\
\hline $\mathrm{H} 63 \mathrm{C}$ & 0.623362 & 0.018380 & 0.202434 & $0.113 *$ & \\
\hline C64 & $0.45758(17)$ & $0.02443(16)$ & $0.29812(11)$ & $0.0366(5)$ & \\
\hline N8 & $0.5048(13)$ & $-0.0503(6)$ & $0.3313(10)$ & $0.041(2)$ & $0.568(16)$ \\
\hline $\mathrm{H} 8$ & 0.529233 & -0.039870 & 0.364177 & $0.049 *$ & $0.568(16)$ \\
\hline C65 & $0.5203(11)$ & $-0.1449(8)$ & $0.320(2)$ & $0.043(2)$ & $0.568(16)$ \\
\hline C66 & $0.4371(11)$ & $-0.1796(9)$ & $0.3306(13)$ & $0.054(3)$ & $0.568(16)$ \\
\hline
\end{tabular}




\begin{tabular}{|c|c|c|c|c|c|}
\hline C67 & $0.4577(10)$ & $-0.2743(8)$ & $0.3258(10)$ & $0.068(3)$ & $0.568(16)$ \\
\hline H67 & 0.402487 & -0.298439 & 0.333925 & $0.081^{*}$ & $0.568(16)$ \\
\hline C68 & $0.5607(12)$ & $-0.3305(7)$ & $0.3090(6)$ & $0.076(4)$ & $0.568(16)$ \\
\hline H68 & 0.575015 & -0.392896 & 0.303746 & $0.091^{*}$ & $0.568(16)$ \\
\hline C69 & $0.6438(11)$ & $-0.2959(8)$ & $0.2996(8)$ & $0.075(3)$ & $0.568(16)$ \\
\hline H69 & 0.712835 & -0.335344 & 0.287442 & $0.090^{*}$ & $0.568(16)$ \\
\hline $\mathrm{C} 70$ & $0.6254(11)$ & $-0.2029(8)$ & $0.3080(13)$ & $0.055(3)$ & $0.568(16)$ \\
\hline $\mathrm{C} 71$ & $0.3243(11)$ & $-0.1204(11)$ & $0.3543(10)$ & $0.065(3)$ & $0.568(16)$ \\
\hline H71A & 0.279197 & -0.151494 & 0.345844 & $0.098^{*}$ & $0.568(16)$ \\
\hline H71B & 0.314728 & -0.064218 & 0.328714 & $0.098^{*}$ & $0.568(16)$ \\
\hline $\mathrm{H} 71 \mathrm{C}$ & 0.306562 & -0.107421 & 0.403678 & $0.098^{*}$ & $0.568(16)$ \\
\hline $\mathrm{C} 72$ & $0.7172(13)$ & $-0.1687(14)$ & $0.2994(12)$ & $0.081(4)$ & $0.568(16)$ \\
\hline $\mathrm{H} 72 \mathrm{~A}$ & 0.702402 & -0.127201 & 0.337189 & $0.121^{*}$ & $0.568(16)$ \\
\hline H72B & 0.728860 & -0.137668 & 0.255390 & $0.121^{*}$ & $0.568(16)$ \\
\hline $\mathrm{H} 72 \mathrm{C}$ & 0.779292 & -0.219388 & 0.300150 & $0.121^{*}$ & $0.568(16)$ \\
\hline $\mathrm{N} 8^{\prime}$ & $0.5095(16)$ & $-0.0569(8)$ & $0.3213(14)$ & $0.042(4)$ & $0.432(16)$ \\
\hline $\mathrm{H} 8^{\prime}$ & 0.556817 & -0.053030 & 0.340151 & $0.050^{*}$ & $0.432(16)$ \\
\hline $\mathrm{C} 65^{\prime}$ & $0.5056(15)$ & $-0.1479(11)$ & $0.322(3)$ & $0.051(3)$ & $0.432(16)$ \\
\hline C66 & $0.4096(14)$ & $-0.1637(10)$ & $0.3363(17)$ & $0.052(3)$ & $0.432(16)$ \\
\hline C67' & $0.4144(14)$ & $-0.2542(11)$ & $0.3297(14)$ & $0.074(4)$ & $0.432(16)$ \\
\hline H67' & 0.352638 & -0.267872 & 0.333863 & $0.089^{*}$ & $0.432(16)$ \\
\hline $\mathrm{C} 68^{\prime}$ & $0.5091(15)$ & $-0.3227(11)$ & $0.3172(9)$ & $0.078(4)$ & $0.432(16)$ \\
\hline H68' & 0.509234 & -0.382652 & 0.315929 & $0.094 *$ & $0.432(16)$ \\
\hline C69' & $0.6055(13)$ & $-0.3071(11)$ & $0.3063(10)$ & $0.073(4)$ & $0.432(16)$ \\
\hline $\mathrm{H} 69^{\prime}$ & 0.668441 & -0.355385 & 0.298997 & $0.088^{*}$ & $0.432(16)$ \\
\hline $\mathrm{C} 70^{\prime}$ & $0.6047(15)$ & $-0.2166(12)$ & $0.3066(19)$ & $0.054(3)$ & $0.432(16)$ \\
\hline $\mathrm{C} 71^{\prime}$ & $0.3032(15)$ & $-0.0927(15)$ & $0.3537(14)$ & $0.070(4)$ & $0.432(16)$ \\
\hline H71D & 0.257377 & -0.112164 & 0.393280 & $0.105^{*}$ & $0.432(16)$ \\
\hline H71E & 0.274080 & -0.084125 & 0.314022 & $0.105^{*}$ & $0.432(16)$ \\
\hline $\mathrm{H} 71 \mathrm{~F}$ & 0.309763 & -0.036180 & 0.365077 & $0.105^{*}$ & $0.432(16)$ \\
\hline $\mathrm{C} 72^{\prime}$ & $0.7029(18)$ & $-0.1920(18)$ & $0.2990(14)$ & $0.070(4)$ & $0.432(16)$ \\
\hline H72D & 0.696102 & -0.134069 & 0.276136 & $0.105^{*}$ & $0.432(16)$ \\
\hline $\mathrm{H} 72 \mathrm{E}$ & 0.762631 & -0.237989 & 0.271407 & $0.105^{*}$ & $0.432(16)$ \\
\hline $\mathrm{H} 72 \mathrm{~F}$ & 0.712747 & -0.188141 & 0.344693 & $0.105^{*}$ & $0.432(16)$ \\
\hline $\mathrm{C} 73$ & $0.46293(18)$ & $0.11139(16)$ & $0.32544(11)$ & $0.0371(5)$ & \\
\hline N9 & $0.54009(16)$ & $0.09251(14)$ & $0.35388(11)$ & $0.0449(5)$ & \\
\hline C74 & $0.5761(2)$ & $0.15374(17)$ & $0.38172(14)$ & $0.0478(6)$ & \\
\hline $\mathrm{C} 75$ & $0.6692(2)$ & $0.1691(2)$ & $0.34225(17)$ & $0.0608(8)$ & \\
\hline $\mathrm{C} 76$ & $0.7139(3)$ & $0.2188(2)$ & $0.3733(2)$ & $0.0768(10)$ & \\
\hline $\mathrm{H} 76$ & 0.775449 & 0.230044 & 0.347570 & $0.092 *$ & \\
\hline $\mathrm{C} 77$ & $0.6687(3)$ & $0.2511(2)$ & $0.4410(2)$ & $0.0815(11)$ & \\
\hline $\mathrm{H} 77$ & 0.699474 & 0.284169 & 0.460721 & $0.098 *$ & \\
\hline $\mathrm{C} 78$ & $0.5783(3)$ & $0.2347(2)$ & $0.47949(18)$ & $0.0690(9)$ & \\
\hline $\mathrm{H} 78$ & 0.547801 & 0.257474 & 0.525202 & $0.083^{*}$ & \\
\hline $\mathrm{C} 79$ & $0.5313(2)$ & $0.18475(18)$ & $0.45160(15)$ & $0.0520(7)$ & \\
\hline $\mathrm{C} 80$ & $0.7237(3)$ & $0.1294(3)$ & $0.26970(19)$ & $0.0857(11)$ & \\
\hline H80A & 0.789078 & 0.142530 & 0.252515 & $0.129 *$ & \\
\hline H80B & 0.679167 & 0.155711 & 0.238954 & $0.129 *$ & \\
\hline
\end{tabular}




\begin{tabular}{|c|c|c|c|c|c|}
\hline $\mathrm{H} 80 \mathrm{C}$ & 0.737762 & 0.064399 & 0.271225 & $0.129 *$ & \\
\hline $\mathrm{C} 81$ & $0.4401(2)$ & $0.1584(2)$ & $0.49680(15)$ & $0.0638(8)$ & \\
\hline H81A & 0.387461 & 0.167967 & 0.471652 & $0.096^{*}$ & \\
\hline H81B & 0.410226 & 0.195206 & 0.538860 & $0.096^{*}$ & \\
\hline $\mathrm{H} 81 \mathrm{C}$ & 0.463872 & 0.095154 & 0.508977 & $0.096^{*}$ & \\
\hline $\mathrm{O} 1$ & $0.9343(13)$ & $0.1372(13)$ & $0.0105(9)$ & $0.228(8)$ & $0.552(19)$ \\
\hline $\mathrm{C} 82$ & $0.8985(19)$ & $0.1455(18)$ & $0.0847(9)$ & $0.180(7)$ & $0.552(19)$ \\
\hline H82A & 0.919797 & 0.086408 & 0.106537 & $0.216^{*}$ & 0.552 (19) \\
\hline H82B & 0.822160 & 0.171249 & 0.099722 & $0.216^{*}$ & $0.552(19)$ \\
\hline $\mathrm{C} 83$ & $0.9497(16)$ & $0.2080(14)$ & $0.1033(8)$ & $0.154(6)$ & $0.552(19)$ \\
\hline H83A & 0.994015 & 0.177455 & 0.134021 & $0.184^{*}$ & $0.552(19)$ \\
\hline H83B & 0.896455 & 0.262114 & 0.127383 & $0.184^{*}$ & $0.552(19)$ \\
\hline C84 & $1.0165(11)$ & $0.2343(12)$ & $0.0345(11)$ & $0.181(6)$ & $0.552(19)$ \\
\hline H84A & 0.981219 & 0.295282 & 0.020252 & $0.217^{*}$ & $0.552(19)$ \\
\hline H84B & 1.085147 & 0.231743 & 0.039001 & $0.217^{*}$ & $0.552(19)$ \\
\hline $\mathrm{C} 85$ & $1.0264(12)$ & $0.1656(19)$ & $-0.0155(9)$ & $0.182(9)$ & $0.552(19)$ \\
\hline H85A & 1.029009 & 0.191379 & -0.062034 & $0.219^{*}$ & $0.552(19)$ \\
\hline H85B & 1.090121 & 0.114196 & -0.017944 & $0.219^{*}$ & $0.552(19)$ \\
\hline $\mathrm{O} 1^{\prime}$ & $0.996(3)$ & $0.2346(19)$ & $0.0573(12)$ & $0.357(16)$ & $0.448(19)$ \\
\hline $\mathrm{C} 82^{\prime}$ & $0.9945(14)$ & $0.2279(10)$ & $-0.0142(9)$ & $0.121(5)$ & $0.448(19)$ \\
\hline $\mathrm{H} 82 \mathrm{C}$ & 1.059140 & 0.231871 & -0.047128 & $0.145^{*}$ & $0.448(19)$ \\
\hline H82D & 0.934419 & 0.274420 & -0.024916 & $0.145^{*}$ & $0.448(19)$ \\
\hline C83' & $0.985(3)$ & $0.1349(14)$ & $-0.0142(11)$ & $0.202(15)$ & 0.448 (19) \\
\hline $\mathrm{H} 83 \mathrm{C}$ & 1.053561 & 0.088211 & -0.020926 & $0.242 *$ & 0.448 (19) \\
\hline H83D & 0.953558 & 0.128606 & -0.050832 & $0.242 *$ & $0.448(19)$ \\
\hline C84' & $0.914(2)$ & $0.1284(19)$ & $0.0577(12)$ & $0.178(10)$ & $0.448(19)$ \\
\hline $\mathrm{H} 84 \mathrm{C}$ & 0.841062 & 0.146918 & 0.056007 & $0.214 *$ & $0.448(19)$ \\
\hline H84D & 0.933465 & 0.066102 & 0.074487 & $0.214^{*}$ & $0.448(19)$ \\
\hline $\mathrm{C} 85^{\prime}$ & $0.928(3)$ & $0.190(2)$ & $0.1051(11)$ & $0.229(16)$ & $0.448(19)$ \\
\hline $\mathrm{H} 85 \mathrm{C}$ & 0.861217 & 0.234413 & 0.128114 & $0.275^{*}$ & $0.448(19)$ \\
\hline H85D & 0.961376 & 0.156266 & 0.140532 & $0.275^{*}$ & $0.448(19)$ \\
\hline $\mathrm{O} 2$ & $0.0069(11)$ & $0.6883(9)$ & $0.2445(8)$ & $0.281(7)$ & $0.692(11)$ \\
\hline $\mathrm{C} 86$ & $0.0053(11)$ & $0.7656(11)$ & $0.2816(7)$ & $0.213(6)$ & $0.692(11)$ \\
\hline H86A & 0.065590 & 0.750775 & 0.301101 & $0.256^{*}$ & $0.692(11)$ \\
\hline H86B & -0.059219 & 0.786982 & 0.318953 & $0.256^{*}$ & $0.692(11)$ \\
\hline $\mathrm{C} 87$ & $0.011(2)$ & $0.8350(10)$ & $0.2237(11)$ & $0.317(14)$ & $0.692(11)$ \\
\hline H87A & 0.015446 & 0.891272 & 0.238782 & $0.380^{*}$ & $0.692(11)$ \\
\hline H87B & -0.045608 & 0.847792 & 0.200871 & $0.380^{*}$ & $0.692(11)$ \\
\hline $\mathrm{C} 88$ & $0.1153(16)$ & $0.7737(13)$ & $0.1806(13)$ & $0.293(12)$ & $0.692(11)$ \\
\hline H88A & 0.169603 & 0.756091 & 0.205728 & $0.351^{*}$ & $0.692(11)$ \\
\hline H88B & 0.140321 & 0.799965 & 0.135616 & $0.351^{*}$ & $0.692(11)$ \\
\hline C89 & $0.0748(13)$ & $0.6963(11)$ & $0.1735(8)$ & $0.232(6)$ & $0.692(11)$ \\
\hline H89A & 0.034344 & 0.710457 & 0.138899 & $0.279^{*}$ & $0.692(11)$ \\
\hline H89B & 0.132786 & 0.640049 & 0.159755 & $0.279^{*}$ & $0.692(11)$ \\
\hline $\mathrm{O} 2^{\prime}$ & $0.0890(18)$ & $0.7946(14)$ & $0.2499(12)$ & $0.198(9)$ & $0.308(11)$ \\
\hline C86' & 0.1048 (19) & $0.733(2)$ & $0.1917(18)$ & 0.239 (19) & $0.308(11)$ \\
\hline $\mathrm{H} 86 \mathrm{C}$ & 0.139241 & 0.670218 & 0.202345 & $0.287^{*}$ & $0.308(11)$ \\
\hline H86D & 0.147548 & 0.749869 & 0.148331 & $0.287 *$ & 0.308 \\
\hline
\end{tabular}




\begin{tabular}{llllll} 
C87' & $-0.007(2)$ & $0.7461(15)$ & $0.1857(16)$ & $0.188(11)$ & $0.308(11)$ \\
H87C & -0.008325 & 0.720700 & 0.142863 & $0.225^{*}$ & $0.308(11)$ \\
H87D & -0.051167 & 0.725750 & 0.226628 & $0.225^{*}$ & $0.308(11)$ \\
C88' & $-0.029(2)$ & $0.8471(15)$ & $0.1836(14)$ & $0.201(11)$ & $0.308(11)$ \\
H88C & -0.097787 & 0.881231 & 0.177046 & $0.241^{*}$ & $0.308(11)$ \\
H88D & 0.026203 & 0.866060 & 0.150442 & $0.241^{*}$ & $0.308(11)$ \\
C89' & $-0.0235(19)$ & $0.846(2)$ & $0.2592(13)$ & $0.210(15)$ & $0.308(11)$ \\
H89C & -0.041572 & 0.906993 & 0.277449 & $0.252^{*}$ & $0.308(11)$ \\
H89D & -0.068652 & 0.814257 & 0.289556 & $0.252^{*}$ & $0.308(11)$ \\
\hline
\end{tabular}

Atomic displacement parameters $\left(\AA^{2}\right)$

\begin{tabular}{|c|c|c|c|c|c|c|}
\hline & $U^{11}$ & $U^{22}$ & $U^{33}$ & $U^{12}$ & $U^{13}$ & $U^{23}$ \\
\hline $\mathrm{Fe} 1$ & $0.0388(2)$ & $0.0358(2)$ & $0.03504(19)$ & $-0.01454(15)$ & $-0.01060(14)$ & $0.00090(14)$ \\
\hline $\mathrm{C} 1$ & $0.0386(13)$ & $0.0378(14)$ & $0.0373(14)$ & $-0.0095(11)$ & $-0.0151(11)$ & $-0.0015(11)$ \\
\hline N1 & $0.045(5)$ & $0.051(5)$ & $0.027(4)$ & $-0.020(3)$ & $-0.015(4)$ & $-0.006(4)$ \\
\hline $\mathrm{C} 2$ & $0.050(4)$ & $0.044(5)$ & $0.038(6)$ & -0.030 & -0.008 & $-0.010(5)$ \\
\hline $\mathrm{C} 3$ & $0.069(4)$ & $0.049(6)$ & $0.042(6)$ & -0.029 & -0.023 & $0.003(5)$ \\
\hline $\mathrm{C} 4$ & $0.104(6)$ & $0.091(8)$ & $0.065(8)$ & $-0.049(6)$ & $-0.020(6)$ & $0.029(6)$ \\
\hline $\mathrm{C} 5$ & $0.099(6)$ & $0.126(11)$ & $0.100(10)$ & $-0.054(7)$ & $0.013(7)$ & $0.034(8)$ \\
\hline C6 & $0.065(5)$ & $0.091(8)$ & $0.101(9)$ & $-0.040(5)$ & $0.006(5)$ & $0.012(7)$ \\
\hline $\mathrm{C} 7$ & $0.055(4)$ & $0.056(6)$ & $0.057(6)$ & $-0.034(4)$ & $-0.011(4)$ & $-0.001(5)$ \\
\hline $\mathrm{C} 8$ & $0.071(6)$ & $0.078(10)$ & $0.059(10)$ & $-0.024(6)$ & $-0.027(5)$ & $0.019(6)$ \\
\hline C9 & $0.059(8)$ & $0.104(10)$ & $0.100(10)$ & $-0.042(7)$ & $-0.003(6)$ & $0.026(8)$ \\
\hline $\mathrm{N} 1^{\prime}$ & $0.040(3)$ & $0.053(4)$ & $0.035(5)$ & $-0.028(3)$ & $-0.012(4)$ & $-0.002(5)$ \\
\hline $\mathrm{C} 2^{\prime}$ & $0.051(4)$ & $0.046(5)$ & $0.030(5)$ & $-0.027(3)$ & $-0.008(3)$ & $-0.006(4)$ \\
\hline $\mathrm{C} 3^{\prime}$ & $0.067(4)$ & $0.054(5)$ & $0.041(6)$ & $-0.034(3)$ & $-0.013(4)$ & $-0.004(4)$ \\
\hline $\mathrm{C} 4^{\prime}$ & $0.097(5)$ & $0.080(7)$ & $0.064(7)$ & $-0.046(5)$ & $-0.020(5)$ & $0.028(5)$ \\
\hline $\mathrm{C} 5^{\prime}$ & $0.081(5)$ & $0.121(10)$ & $0.095(9)$ & $-0.061(6)$ & $-0.002(6)$ & $0.045(7)$ \\
\hline $\mathrm{C} 6^{\prime}$ & $0.057(4)$ & $0.098(9)$ & $0.088(8)$ & $-0.038(5)$ & $-0.004(4)$ & $0.018(6)$ \\
\hline $\mathrm{C} 7^{\prime}$ & $0.047(4)$ & $0.065(6)$ & $0.055(5)$ & $-0.031(4)$ & $-0.011(3)$ & $-0.007(4)$ \\
\hline $\mathrm{C} 8^{\prime}$ & $0.094(7)$ & $0.067(7)$ & $0.074(12)$ & $-0.027(5)$ & $-0.050(6)$ & $0.027(6)$ \\
\hline $\mathrm{C} 9^{\prime}$ & $0.047(7)$ & $0.093(8)$ & $0.101(9)$ & $-0.016(6)$ & $-0.032(8)$ & $0.035(7)$ \\
\hline $\mathrm{C} 10$ & $0.0408(14)$ & $0.0392(14)$ & $0.0499(15)$ & $-0.0104(11)$ & $-0.0168(12)$ & $0.0030(12)$ \\
\hline $\mathrm{N} 2$ & $0.0618(14)$ & $0.0461(13)$ & $0.0544(14)$ & $-0.0100(11)$ & $-0.0253(12)$ & $-0.0097(11)$ \\
\hline $\mathrm{C} 11$ & $0.0696(19)$ & $0.0483(17)$ & $0.0621(19)$ & $0.0020(15)$ & $-0.0377(17)$ & $-0.0142(14)$ \\
\hline $\mathrm{C} 12$ & $0.094(2)$ & $0.0451(18)$ & $0.098(3)$ & $-0.0025(17)$ & $-0.062(2)$ & $-0.0181(17)$ \\
\hline $\mathrm{C} 13$ & $0.114(3)$ & $0.074(3)$ & $0.145(4)$ & $0.000(2)$ & $-0.071(3)$ & $-0.041(3)$ \\
\hline C14 & $0.114(4)$ & $0.092(3)$ & $0.122(4)$ & $0.013(3)$ & $-0.065(3)$ & $-0.060(3)$ \\
\hline $\mathrm{C} 15$ & $0.087(3)$ & $0.110(3)$ & $0.077(3)$ & $0.027(2)$ & $-0.038(2)$ & $-0.034(2)$ \\
\hline $\mathrm{C} 16$ & $0.070(2)$ & $0.073(2)$ & $0.063(2)$ & $0.0087(16)$ & $-0.0324(18)$ & $-0.0187(17)$ \\
\hline $\mathrm{C} 17$ & $0.109(3)$ & $0.081(3)$ & $0.123(3)$ & $-0.043(2)$ & $-0.054(3)$ & $0.015(2)$ \\
\hline $\mathrm{C} 18$ & $0.070(2)$ & $0.113(3)$ & $0.063(2)$ & $-0.008(2)$ & $-0.0109(17)$ & $0.000(2)$ \\
\hline C19 & $0.0455(15)$ & $0.0394(14)$ & $0.0482(15)$ & $-0.0151(12)$ & $-0.0165(12)$ & $0.0030(12)$ \\
\hline N3 & $0.0548(14)$ & $0.0583(15)$ & $0.0595(15)$ & $-0.0268(12)$ & $-0.0084(12)$ & $0.0162(12)$ \\
\hline $\mathrm{C} 20$ & $0.0568(18)$ & $0.064(2)$ & $0.076(2)$ & $-0.0296(16)$ & $-0.0067(16)$ & $0.0218(16)$ \\
\hline $\mathrm{C} 21$ & $0.096(3)$ & $0.101(3)$ & $0.072(2)$ & $-0.045(2)$ & $-0.004(2)$ & $0.027(2)$ \\
\hline $\mathrm{C} 22$ & $0.175(5)$ & $0.154(5)$ & 0.089 & $-0.086(4)$ & $0.003(3)$ & $0.037(3)$ \\
\hline
\end{tabular}




\begin{tabular}{|c|c|c|c|c|c|c|}
\hline $\mathrm{C} 23$ & $0.167(6)$ & $0.176(6)$ & $0.146(5)$ & $-0.114(5)$ & $-0.006(4)$ & $0.045(5)$ \\
\hline $\mathrm{C} 24$ & $0.124(4)$ & $0.136(4)$ & $0.157(5)$ & $-0.095(3)$ & $-0.038(4)$ & $0.039(4)$ \\
\hline $\mathrm{C} 25$ & $0.093(3)$ & $0.092(3)$ & $0.116(3)$ & $-0.056(2)$ & $-0.031(2)$ & $0.026(2)$ \\
\hline $\mathrm{C} 26$ & $0.185(5)$ & $0.134(4)$ & $0.085(3)$ & $-0.081(4)$ & $-0.037(3)$ & $0.012(3)$ \\
\hline $\mathrm{C} 27$ & $0.176(5)$ & $0.120(4)$ & $0.148(5)$ & $-0.083(4)$ & $-0.069(4)$ & $0.005(3)$ \\
\hline $\mathrm{C} 28$ & $0.0436(14)$ & $0.0416(15)$ & $0.0379(13)$ & $-0.0155(12)$ & $-0.0112(11)$ & $0.0030(11)$ \\
\hline $\mathrm{N} 4$ & $0.0595(14)$ & $0.0379(13)$ & $0.0616(14)$ & $-0.0123(11)$ & $-0.0306(12)$ & $0.0032(10)$ \\
\hline $\mathrm{C} 29$ & $0.0577(17)$ & $0.0364(15)$ & $0.074(2)$ & $-0.0072(13)$ & $-0.0371(15)$ & $0.0009(14)$ \\
\hline $\mathrm{C} 30$ & $0.071(2)$ & $0.0430(17)$ & $0.089(2)$ & $-0.0169(15)$ & $-0.0421(18)$ & $0.0078(15)$ \\
\hline C31 & $0.094(3)$ & $0.052(2)$ & 0.120 & -0.0301 (19) & $-0.052(2)$ & $0.005(2)$ \\
\hline $\mathrm{C} 32$ & $0.107(3)$ & $0.056(2)$ & $0.128(4)$ & $-0.022(2)$ & $-0.058(3)$ & $-0.017(2)$ \\
\hline $\mathrm{C} 33$ & $0.084(2)$ & $0.059(2)$ & $0.088(2)$ & $-0.0017(18)$ & $-0.039(2)$ & $-0.0197(18)$ \\
\hline $\mathrm{C} 34$ & $0.0621(18)$ & $0.0437(16)$ & $0.077(2)$ & $-0.0026(14)$ & $-0.0342(17)$ & $-0.0060(15)$ \\
\hline $\mathrm{C} 35$ & $0.102(3)$ & $0.069(2)$ & $0.097(3)$ & $-0.035(2)$ & $-0.029(2)$ & $0.014(2)$ \\
\hline $\mathrm{C} 36$ & $0.069(2)$ & $0.070(2)$ & $0.083(2)$ & $-0.0125(18)$ & $-0.0158(18)$ & $-0.0082(18)$ \\
\hline $\mathrm{C} 37$ & $0.0437(14)$ & $0.0391(14)$ & $0.0364(13)$ & $-0.0140(11)$ & $-0.0115(11)$ & $0.0033(11)$ \\
\hline N5 & $0.0586(14)$ & $0.0451(13)$ & $0.0492(13)$ & $-0.0113(11)$ & $-0.0275(11)$ & $0.0027(10)$ \\
\hline C38 & 0.0644 (19) & $0.0472(17)$ & $0.0592(18)$ & $-0.0090(14)$ & $-0.0372(15)$ & $0.0014(14)$ \\
\hline $\mathrm{C} 39$ & $0.065(2)$ & $0.061(2)$ & $0.083(2)$ & $-0.0084(17)$ & $-0.0372(18)$ & $-0.0034(17)$ \\
\hline $\mathrm{C} 40$ & $0.077(3)$ & $0.077(3)$ & $0.133(4)$ & $0.002(2)$ & $-0.049(3)$ & $-0.001(3)$ \\
\hline $\mathrm{C} 41$ & $0.122(4)$ & $0.069(3)$ & $0.168(5)$ & $0.010(3)$ & $-0.084(4)$ & $0.023(3)$ \\
\hline $\mathrm{C} 42$ & $0.140(4)$ & $0.066(3)$ & 0.119 & -0.019 (3) & $-0.073(3)$ & 0.033 \\
\hline $\mathrm{C} 43$ & $0.094(2)$ & $0.0542(19)$ & $0.066(2)$ & $-0.0192(18)$ & $-0.0384(19)$ & $0.0095(16)$ \\
\hline $\mathrm{C} 44$ & $0.078(2)$ & $0.096(3)$ & $0.094(3)$ & $-0.018(2)$ & $-0.012(2)$ & $-0.002(2)$ \\
\hline $\mathrm{C} 45$ & $0.133(4)$ & $0.079(3)$ & $0.074(2)$ & $-0.038(2)$ & $-0.016(2)$ & $0.013(2)$ \\
\hline $\mathrm{C} 46$ & $0.0359(12)$ & $0.0412(14)$ & $0.0308(12)$ & $-0.0159(11)$ & $-0.0067(10)$ & $0.0050(10)$ \\
\hline N6 & $0.0468(12)$ & $0.0354(11)$ & $0.0381(11)$ & $-0.0141(9)$ & $-0.0172(9)$ & $0.0030(9)$ \\
\hline $\mathrm{C} 47$ & $0.0604(17)$ & $0.0382(14)$ & $0.0520(16)$ & $-0.0130(13)$ & $-0.0311(13)$ & $0.0006(12)$ \\
\hline $\mathrm{C} 48$ & $0.070(2)$ & $0.0504(17)$ & $0.080(2)$ & $-0.0257(15)$ & $-0.0369(17)$ & $0.0079(15)$ \\
\hline C49 & $0.110(3)$ & $0.075(3)$ & $0.130(4)$ & $-0.050(2)$ & $-0.064(3)$ & $0.009(2)$ \\
\hline $\mathrm{C} 50$ & $0.157(4)$ & $0.078(3)$ & $0.131(4)$ & $-0.047(3)$ & $-0.092(4)$ & $-0.004(3)$ \\
\hline $\mathrm{C} 51$ & $0.135(3)$ & $0.068(2)$ & $0.071(2)$ & $-0.013(2)$ & $-0.062(2)$ & $-0.0150(18)$ \\
\hline $\mathrm{C} 52$ & $0.081(2)$ & $0.0481(17)$ & $0.0518(17)$ & $-0.0080(15)$ & $-0.0346(16)$ & $-0.0027(13)$ \\
\hline C53 & $0.072(2)$ & $0.076(2)$ & $0.095(3)$ & $-0.0385(18)$ & $-0.0188(19)$ & $0.0206(19)$ \\
\hline $\mathrm{C} 54$ & $0.099(3)$ & $0.076(2)$ & $0.0475(18)$ & $-0.011(2)$ & $-0.0131(17)$ & $-0.0015(16)$ \\
\hline $\mathrm{C} 55$ & $0.0414(13)$ & $0.0350(13)$ & $0.0382(13)$ & $-0.0137(11)$ & $-0.0110(11)$ & $-0.0014(10)$ \\
\hline N7 & $0.0504(14)$ & $0.0394(12)$ & $0.0521(13)$ & $-0.0082(11)$ & $-0.0205(11)$ & $-0.0082(10)$ \\
\hline $\mathrm{C} 56$ & $0.0673(19)$ & $0.0462(16)$ & $0.0334(14)$ & $-0.0023(14)$ & $-0.0184(13)$ & $-0.0028(12)$ \\
\hline $\mathrm{C} 57$ & $0.101(2)$ & $0.0456(17)$ & $0.0453(16)$ & $-0.0033(17)$ & $-0.0291(17)$ & $-0.0051(13)$ \\
\hline $\mathrm{C} 58$ & $0.132(4)$ & $0.056(2)$ & $0.062(2)$ & $0.012(2)$ & $-0.034(2)$ & $-0.0182(17)$ \\
\hline $\mathrm{C} 59$ & $0.107(3)$ & $0.096(3)$ & $0.070(3)$ & $0.038(3)$ & $-0.032(2)$ & $-0.023(2)$ \\
\hline $\mathrm{C} 60$ & $0.072(2)$ & $0.120(3)$ & $0.053(2)$ & $0.008(2)$ & $-0.0155(17)$ & $-0.014(2)$ \\
\hline C61 & $0.0622(19)$ & $0.076(2)$ & $0.0361(15)$ & $-0.0035(17)$ & $-0.0137(14)$ & $-0.0047(14)$ \\
\hline C62 & $0.138(3)$ & $0.0514(19)$ & $0.071(2)$ & $-0.033(2)$ & $-0.039(2)$ & $-0.0058(16)$ \\
\hline C63 & $0.063(2)$ & $0.109(3)$ & $0.0546(19)$ & $-0.0346(19)$ & $-0.0045(15)$ & $-0.0038(18)$ \\
\hline C64 & $0.0344(12)$ & $0.0390(14)$ & $0.0344(13)$ & $-0.0117(11)$ & $-0.0065(10)$ & $0.0018(10)$ \\
\hline N8 & $0.059(4)$ & $0.029(3)$ & $0.035(5)$ & $-0.013(3)$ & $-0.015(3)$ & $0.001(3)$ \\
\hline C65 & $0.063(4)$ & $0.037(4)$ & $0.035(4)$ & $-0.021(3)$ & $-0.017(5)$ & $0.005(3)$ \\
\hline
\end{tabular}




\begin{tabular}{|c|c|c|c|c|c|c|}
\hline C66 & $0.073(6)$ & $0.046(4)$ & $0.040(4)$ & $-0.018(4)$ & $-0.012(6)$ & $0.000(4)$ \\
\hline C67 & $0.092(10)$ & $0.046(6)$ & $0.079(4)$ & $-0.029(6)$ & $-0.039(8)$ & $0.014(6)$ \\
\hline C68 & $0.104(10)$ & $0.039(4)$ & $0.087(4)$ & $-0.020(5)$ & $-0.035(7)$ & $0.008(3)$ \\
\hline C69 & $0.098(7)$ & $0.044(4)$ & $0.076(4)$ & $-0.007(5)$ & $-0.033(6)$ & $0.003(3)$ \\
\hline C70 & $0.075(6)$ & $0.040(4)$ & $0.050(3)$ & -0.009 (4) & $-0.030(6)$ & $0.001(4)$ \\
\hline C71 & $0.070(7)$ & $0.068(9)$ & $0.070(5)$ & $-0.037(6)$ & $-0.021(5)$ & $0.005(6)$ \\
\hline $\mathrm{C} 72$ & $0.051(5)$ & $0.084(11)$ & $0.094(7)$ & $-0.001(5)$ & $-0.024(4)$ & $0.004(6)$ \\
\hline N8' & $0.037(4)$ & $0.054(6)$ & $0.039(7)$ & $-0.011(4)$ & $-0.019(4)$ & $-0.004(4)$ \\
\hline C65' & $0.073(7)$ & $0.034(5)$ & $0.043(5)$ & -0.015 (4) & $-0.016(7)$ & $0.004(5)$ \\
\hline C66' & $0.071(7)$ & $0.047(6)$ & $0.048(6)$ & $-0.034(6)$ & $-0.017(8)$ & $0.006(6)$ \\
\hline C67' & $0.104(10)$ & $0.058(7)$ & $0.075(5)$ & $-0.032(7)$ & $-0.042(10)$ & $0.013(6)$ \\
\hline C68' & $0.108(11)$ & $0.045(6)$ & $0.090(6)$ & $-0.025(7)$ & $-0.041(8)$ & $0.007(5)$ \\
\hline C69' & $0.093(10)$ & $0.043(6)$ & $0.084(5)$ & $-0.014(6)$ & $-0.033(8)$ & $0.003(5)$ \\
\hline $\mathrm{C} 70^{\prime}$ & $0.078(6)$ & $0.042(5)$ & $0.055(4)$ & $-0.021(4)$ & $-0.034(5)$ & $-0.002(5)$ \\
\hline $\mathrm{C} 71^{\prime}$ & $0.063(7)$ & $0.065(11)$ & $0.076(7)$ & $-0.029(6)$ & $-0.002(6)$ & $0.008(8)$ \\
\hline $\mathrm{C} 72^{\prime}$ & $0.071(9)$ & $0.066(10)$ & $0.065(6)$ & $0.002(7)$ & $-0.034(7)$ & $0.001(6)$ \\
\hline C73 & $0.0369(13)$ & $0.0428(14)$ & $0.0319(12)$ & -0.0157 (11) & $-0.0068(10)$ & $0.0034(10)$ \\
\hline N9 & $0.0461(12)$ & $0.0450(12)$ & $0.0494(12)$ & $-0.0170(10)$ & $-0.0198(10)$ & $0.0033(10)$ \\
\hline $\mathrm{C} 74$ & $0.0513(16)$ & $0.0419(15)$ & $0.0604(17)$ & $-0.0159(12)$ & $-0.0324(13)$ & $0.0101(12)$ \\
\hline $\mathrm{C} 75$ & $0.0538(17)$ & $0.0613(19)$ & $0.077(2)$ & $-0.0240(15)$ & $-0.0286(16)$ & $0.0111(16)$ \\
\hline $\mathrm{C} 76$ & $0.065(2)$ & $0.071(2)$ & $0.114(3)$ & $-0.0345(18)$ & $-0.043(2)$ & $0.017(2)$ \\
\hline $\mathrm{C} 77$ & $0.093(3)$ & $0.057(2)$ & 0.125 & $-0.0309(19)$ & $-0.071(3)$ & $0.007(2)$ \\
\hline $\mathrm{C} 78$ & $0.086(2)$ & $0.0534(19)$ & $0.082(2)$ & $-0.0179(17)$ & $-0.0511(19)$ & $-0.0015(16)$ \\
\hline C79 & $0.0629(17)$ & $0.0422(15)$ & $0.0589(17)$ & $-0.0137(13)$ & $-0.0345(14)$ & $0.0063(13)$ \\
\hline $\mathrm{C} 80$ & $0.061(2)$ & $0.107(3)$ & $0.093(3)$ & $-0.038(2)$ & $-0.0128(19)$ & $0.005(2)$ \\
\hline $\mathrm{C} 81$ & $0.074(2)$ & $0.065(2)$ & $0.0521(17)$ & $-0.0163(16)$ & $-0.0250(15)$ & $0.0024(14)$ \\
\hline $\mathrm{O} 1$ & $0.217(14)$ & $0.334(16)$ & $0.126(12)$ & $-0.104(11)$ & $0.013(12)$ & $-0.093(12)$ \\
\hline $\mathrm{C} 82$ & $0.171(14)$ & $0.232(19)$ & $0.118(13)$ & $-0.045(13)$ & $-0.007(11)$ & $-0.078(12)$ \\
\hline $\mathrm{C} 83$ & $0.163(12)$ & $0.159(14)$ & $0.130(9)$ & $0.007(10)$ & $-0.092(9)$ & $-0.008(9)$ \\
\hline $\mathrm{C} 84$ & $0.109(9)$ & $0.251(17)$ & $0.169(13)$ & $-0.009(9)$ & $-0.076(9)$ & $0.008(10)$ \\
\hline $\mathrm{C} 85$ & $0.112(9)$ & $0.28(3)$ & $0.113(10)$ & $-0.007(14)$ & $-0.023(8)$ & $-0.016(14)$ \\
\hline $\mathrm{O} 1^{\prime}$ & $0.59(4)$ & $0.44(3)$ & $0.205(18)$ & $-0.32(3)$ & $-0.17(2)$ & $0.000(17)$ \\
\hline C82' & $0.113(11)$ & $0.137(11)$ & $0.129(10)$ & $-0.035(9)$ & $-0.063(9)$ & $0.004(9)$ \\
\hline C83' & $0.26(4)$ & $0.199(17)$ & $0.121(14)$ & $-0.11(2)$ & $0.047(18)$ & $-0.035(13)$ \\
\hline $\mathrm{C} 84^{\prime}$ & $0.23(2)$ & $0.175(18)$ & $0.087(16)$ & $-0.052(15)$ & $0.024(17)$ & $-0.031(15)$ \\
\hline $\mathrm{C} 85^{\prime}$ & $0.31(4)$ & $0.20(3)$ & $0.123(14)$ & $-0.01(2)$ & $-0.050(18)$ & $-0.050(14)$ \\
\hline $\mathrm{O} 2$ & $0.221(10)$ & $0.248(12)$ & $0.376(17)$ & $-0.092(10)$ & $-0.084(10)$ & $0.083(11)$ \\
\hline $\mathrm{C} 86$ & $0.142(9)$ & $0.235(15)$ & $0.243(13)$ & $-0.042(11)$ & $-0.066(9)$ & $0.092(10)$ \\
\hline $\mathrm{C} 87$ & $0.44(4)$ & $0.198(16)$ & $0.30(3)$ & $-0.09(2)$ & $-0.13(2)$ & $0.120(13)$ \\
\hline C88 & $0.35(3)$ & $0.33(2)$ & $0.27(2)$ & $-0.25(2)$ & $-0.048(16)$ & 0.109 (17) \\
\hline C89 & $0.236(18)$ & $0.226(14)$ & $0.284(14)$ & $-0.091(12)$ & $-0.142(12)$ & $0.060(12)$ \\
\hline $\mathrm{O} 2^{\prime}$ & 0.235 (19) & $0.166(17)$ & $0.200(19)$ & $-0.070(14)$ & $-0.082(17)$ & $0.070(13)$ \\
\hline $\mathrm{C} 86^{\prime}$ & $0.23(2)$ & $0.22(3)$ & $0.29(4)$ & $-0.02(2)$ & $-0.17(3)$ & $0.02(2)$ \\
\hline $\mathrm{C} 87^{\prime}$ & $0.17(2)$ & $0.153(16)$ & $0.21(3)$ & $0.026(15)$ & $-0.092(19)$ & $0.015(16)$ \\
\hline C88' & $0.25(3)$ & $0.178(16)$ & $0.184(19)$ & $-0.053(17)$ & $-0.11(2)$ & 0.057 (19) \\
\hline C89' & $0.23(2)$ & $0.26(4)$ & $0.16(2)$ & $-0.09(2)$ & $-0.084(19)$ & $0.02(2)$ \\
\hline
\end{tabular}


Geometric parameters $\left(\AA,{ }^{\circ}\right)$

\begin{tabular}{|c|c|c|c|}
\hline $\mathrm{Fe} 1-\mathrm{C} 10$ & $1.851(3)$ & $\mathrm{C} 53-\mathrm{H} 53 \mathrm{C}$ & 0.9600 \\
\hline $\mathrm{Fe} 1-\mathrm{C} 1$ & $1.852(2)$ & C54-H54A & 0.9600 \\
\hline $\mathrm{Fe} 1-\mathrm{C} 19$ & $1.854(3)$ & С54-H54B & 0.9600 \\
\hline $\mathrm{Fe} 1-\mathrm{C} 46$ & $1.955(2)$ & $\mathrm{C} 54-\mathrm{H} 54 \mathrm{C}$ & 0.9600 \\
\hline $\mathrm{Fe} 1-\mathrm{C} 73$ & $2.011(2)$ & $\mathrm{C} 55-\mathrm{C} 64$ & $1.390(3)$ \\
\hline $\mathrm{Fe} 1-\mathrm{C} 28$ & $2.014(2)$ & $\mathrm{C} 55-\mathrm{N} 7$ & $1.418(3)$ \\
\hline $\mathrm{C} 1-\mathrm{N} 1^{\prime}$ & $1.177(6)$ & $\mathrm{N} 7-\mathrm{C} 56$ & $1.403(3)$ \\
\hline $\mathrm{C} 1-\mathrm{N} 1$ & $1.178(7)$ & $\mathrm{N} 7-\mathrm{H} 7$ & $0.85(3)$ \\
\hline $\mathrm{N} 1-\mathrm{C} 2$ & $1.407(10)$ & $\mathrm{C} 56-\mathrm{C} 61$ & $1.405(4)$ \\
\hline $\mathrm{C} 2-\mathrm{C} 3$ & $1.388(10)$ & $\mathrm{C} 56-\mathrm{C} 57$ & $1.408(4)$ \\
\hline $\mathrm{C} 2-\mathrm{C} 7$ & $1.401(11)$ & $\mathrm{C} 57-\mathrm{C} 58$ & $1.388(5)$ \\
\hline $\mathrm{C} 3-\mathrm{C} 4$ & $1.381(11)$ & $\mathrm{C} 57-\mathrm{C} 62$ & $1.500(5)$ \\
\hline $\mathrm{C} 3-\mathrm{C} 8$ & $1.506(8)$ & $\mathrm{C} 58-\mathrm{C} 59$ & $1.367(6)$ \\
\hline $\mathrm{C} 4-\mathrm{C} 5$ & $1.365(12)$ & $\mathrm{C} 58-\mathrm{H} 58$ & 0.9300 \\
\hline $\mathrm{C} 4-\mathrm{H} 4$ & 0.9300 & $\mathrm{C} 59-\mathrm{C} 60$ & $1.370(6)$ \\
\hline $\mathrm{C} 5-\mathrm{C} 6$ & $1.363(12)$ & C59-H59 & 0.9300 \\
\hline $\mathrm{C} 5-\mathrm{H} 5$ & 0.9300 & $\mathrm{C} 60-\mathrm{C} 61$ & $1.393(4)$ \\
\hline $\mathrm{C} 6-\mathrm{C} 7$ & $1.376(11)$ & $\mathrm{C} 60-\mathrm{H} 60$ & 0.9300 \\
\hline $\mathrm{C} 6-\mathrm{H} 6$ & 0.9300 & $\mathrm{C} 61-\mathrm{C} 63$ & $1.497(4)$ \\
\hline $\mathrm{C} 7-\mathrm{C} 9$ & $1.513(8)$ & C62-H62A & 0.9600 \\
\hline $\mathrm{C} 8-\mathrm{H} 8 \mathrm{~A}$ & 0.9600 & C62-H62B & 0.9600 \\
\hline $\mathrm{C} 8-\mathrm{H} 8 \mathrm{~B}$ & 0.9600 & C62-H62C & 0.9600 \\
\hline $\mathrm{C} 8-\mathrm{H} 8 \mathrm{C}$ & 0.9600 & C63-H63A & 0.9600 \\
\hline C9-H9A & 0.9600 & С63-H63В & 0.9600 \\
\hline C9-H9B & 0.9600 & C63-H63C & 0.9600 \\
\hline C9- $\mathrm{H} 9 \mathrm{C}$ & 0.9600 & $\mathrm{C} 64-\mathrm{N} 8^{\prime}$ & $1.345(7)$ \\
\hline $\mathrm{N} 1^{\prime}-\mathrm{C} 2^{\prime}$ & $1.406(9)$ & $\mathrm{C} 64-\mathrm{N} 8$ & $1.348(6)$ \\
\hline $\mathrm{C} 2^{\prime}-\mathrm{C} 3^{\prime}$ & $1.387(10)$ & $\mathrm{C} 64-\mathrm{C} 73$ & $1.523(3)$ \\
\hline $\mathrm{C} 2{ }^{\prime}-\mathrm{C} 7^{\prime}$ & $1.401(10)$ & $\mathrm{N} 8-\mathrm{C} 65$ & $1.438(10)$ \\
\hline $\mathrm{C} 3^{\prime}-\mathrm{C} 4^{\prime}$ & $1.382(10)$ & $\mathrm{N} 8-\mathrm{H} 8$ & 0.8600 \\
\hline $\mathrm{C} 3^{\prime}-\mathrm{C} 8^{\prime}$ & $1.508(8)$ & $\mathrm{C} 65-\mathrm{C} 66$ & $1.387(10)$ \\
\hline $\mathrm{C} 4^{\prime}-\mathrm{C} 5^{\prime}$ & $1.368(11)$ & $\mathrm{C} 65-\mathrm{C} 70$ & $1.408(10)$ \\
\hline $\mathrm{C} 4^{\prime}-\mathrm{H} 4^{\prime}$ & 0.9300 & $\mathrm{C} 66-\mathrm{C} 67$ & $1.405(10)$ \\
\hline $\mathrm{C} 5^{\prime}-\mathrm{C} 6^{\prime}$ & $1.364(10)$ & $\mathrm{C} 66-\mathrm{C} 71$ & $1.497(7)$ \\
\hline $\mathrm{C}^{\prime}-\mathrm{H} 5^{\prime}$ & 0.9300 & $\mathrm{C} 67-\mathrm{C} 68$ & $1.373(11)$ \\
\hline $\mathrm{C} 6^{\prime}-\mathrm{C} 7^{\prime}$ & $1.364(10)$ & C67-H67 & 0.9300 \\
\hline $\mathrm{C} 6^{\prime}-\mathrm{H} 6^{\prime}$ & 0.9300 & $\mathrm{C} 68-\mathrm{C} 69$ & $1.387(11)$ \\
\hline $\mathrm{C} 7^{\prime}-\mathrm{C} 9^{\prime}$ & $1.511(8)$ & $\mathrm{C} 68-\mathrm{H} 68$ & 0.9300 \\
\hline $\mathrm{C} 8^{\prime}-\mathrm{H} 8 \mathrm{D}$ & 0.9600 & $\mathrm{C} 69-\mathrm{C} 70$ & $1.394(10)$ \\
\hline $\mathrm{C} 8^{\prime}-\mathrm{H} 8 \mathrm{E}$ & 0.9600 & C69-H69 & 0.9300 \\
\hline $\mathrm{C} 8^{\prime}-\mathrm{H} 8 \mathrm{~F}$ & 0.9600 & $\mathrm{C} 70-\mathrm{C} 72$ & $1.498(7)$ \\
\hline C9'-H9D & 0.9600 & $\mathrm{C} 71-\mathrm{H} 71 \mathrm{~A}$ & 0.9600 \\
\hline C9'-H9E & 0.9600 & $\mathrm{C} 71-\mathrm{H} 71 \mathrm{~B}$ & 0.9600 \\
\hline C9'-H9F & 0.9600 & $\mathrm{C} 71-\mathrm{H} 71 \mathrm{C}$ & 0.9600 \\
\hline $\mathrm{C} 10-\mathrm{N} 2$ & $1.172(3)$ & $\mathrm{C} 72-\mathrm{H} 72 \mathrm{~A}$ & 0.9600 \\
\hline $\mathrm{N} 2-\mathrm{C} 11$ & $1.386(3)$ & $\mathrm{C} 72-\mathrm{H} 72 \mathrm{~B}$ & 0.9600 \\
\hline
\end{tabular}




\begin{tabular}{|c|c|c|c|}
\hline $\mathrm{C} 11-\mathrm{C} 16$ & $1.369(4)$ & $\mathrm{C} 72-\mathrm{H} 72 \mathrm{C}$ & 0.9600 \\
\hline $\mathrm{C} 11-\mathrm{C} 12$ & $1.429(4)$ & $\mathrm{N} 8^{\prime}-\mathrm{C} 65^{\prime}$ & $1.428(12)$ \\
\hline $\mathrm{C} 12-\mathrm{C} 13$ & $1.369(5)$ & $\mathrm{N} 8^{\prime}-\mathrm{H} 8^{\prime}$ & 0.8600 \\
\hline $\mathrm{C} 12-\mathrm{C} 17$ & $1.483(5)$ & $\mathrm{C} 65^{\prime}-\mathrm{C} 66^{\prime}$ & $1.384(12)$ \\
\hline $\mathrm{C} 13-\mathrm{C} 14$ & $1.338(6)$ & $\mathrm{C} 65^{\prime}-\mathrm{C} 70^{\prime}$ & $1.407(12)$ \\
\hline $\mathrm{C} 13-\mathrm{H} 13$ & 0.9300 & $\mathrm{C} 66^{\prime}-\mathrm{C} 67^{\prime}$ & $1.396(12)$ \\
\hline $\mathrm{C} 14-\mathrm{C} 15$ & $1.393(6)$ & $\mathrm{C} 66^{\prime}-\mathrm{C} 71^{\prime}$ & $1.493(8)$ \\
\hline $\mathrm{C} 14-\mathrm{H} 14$ & 0.9300 & $\mathrm{C} 67^{\prime}-\mathrm{C} 68^{\prime}$ & $1.367(13)$ \\
\hline $\mathrm{C} 15-\mathrm{C} 16$ & $1.424(5)$ & C67'-H67' & 0.9300 \\
\hline $\mathrm{C} 15-\mathrm{H} 15$ & 0.9300 & $\mathrm{C} 68^{\prime}-\mathrm{C} 69^{\prime}$ & $1.394(14)$ \\
\hline $\mathrm{C} 16-\mathrm{C} 18$ & $1.493(5)$ & C68 $-\mathrm{H} 68^{\prime}$ & 0.9300 \\
\hline C17-H17A & 0.9600 & $\mathrm{C} 69^{\prime}-\mathrm{C} 70^{\prime}$ & $1.399(13)$ \\
\hline C17-H17B & 0.9600 & $\mathrm{C} 69^{\prime}-\mathrm{H} 69^{\prime}$ & 0.9300 \\
\hline $\mathrm{C} 17-\mathrm{H} 17 \mathrm{C}$ & 0.9600 & $\mathrm{C} 70^{\prime}-\mathrm{C} 72^{\prime}$ & $1.499(8)$ \\
\hline $\mathrm{C} 18-\mathrm{H} 18 \mathrm{~A}$ & 0.9600 & $\mathrm{C} 71^{\prime}-\mathrm{H} 71 \mathrm{D}$ & 0.9600 \\
\hline C18-H18B & 0.9600 & $\mathrm{C} 71^{\prime}-\mathrm{H} 71 \mathrm{E}$ & 0.9600 \\
\hline $\mathrm{C} 18-\mathrm{H} 18 \mathrm{C}$ & 0.9600 & $\mathrm{C} 71^{\prime}-\mathrm{H} 71 \mathrm{~F}$ & 0.9600 \\
\hline $\mathrm{C} 19-\mathrm{N} 3$ & $1.170(3)$ & $\mathrm{C} 72^{\prime}-\mathrm{H} 72 \mathrm{D}$ & 0.9600 \\
\hline $\mathrm{N} 3-\mathrm{C} 20$ & $1.411(3)$ & $\mathrm{C} 72^{\prime}-\mathrm{H} 72 \mathrm{E}$ & 0.9600 \\
\hline $\mathrm{C} 20-\mathrm{C} 25$ & $1.374(5)$ & $\mathrm{C} 72^{\prime}-\mathrm{H} 72 \mathrm{~F}$ & 0.9600 \\
\hline $\mathrm{C} 20-\mathrm{C} 21$ & $1.404(5)$ & $\mathrm{C} 73-\mathrm{N} 9$ & 1.285 \\
\hline $\mathrm{C} 21-\mathrm{C} 22$ & $1.378(5)$ & N9-C74 & $1.415(3)$ \\
\hline $\mathrm{C} 21-\mathrm{C} 26$ & $1.487(5)$ & $\mathrm{C} 74-\mathrm{C} 75$ & $1.398(4)$ \\
\hline $\mathrm{C} 22-\mathrm{C} 23$ & $1.339(7)$ & $\mathrm{C} 74-\mathrm{C} 79$ & $1.403(4)$ \\
\hline $\mathrm{C} 22-\mathrm{H} 22$ & 0.9300 & $\mathrm{C} 75-\mathrm{C} 76$ & $1.398(4)$ \\
\hline $\mathrm{C} 23-\mathrm{C} 24$ & $1.351(7)$ & $\mathrm{C} 75-\mathrm{C} 80$ & $1.501(4)$ \\
\hline $\mathrm{C} 23-\mathrm{H} 23$ & 0.9300 & $\mathrm{C} 76-\mathrm{C} 77$ & $1.371(5)$ \\
\hline $\mathrm{C} 24-\mathrm{C} 25$ & $1.426(6)$ & C76-H76 & 0.9300 \\
\hline $\mathrm{C} 24-\mathrm{H} 24$ & 0.9300 & $\mathrm{C} 77-\mathrm{C} 78$ & $1.368(5)$ \\
\hline $\mathrm{C} 25-\mathrm{C} 27$ & $1.483(6)$ & C77-H77 & 0.9300 \\
\hline $\mathrm{C} 26-\mathrm{H} 26 \mathrm{~A}$ & 0.9600 & $\mathrm{C} 78-\mathrm{C} 79$ & $1.390(4)$ \\
\hline $\mathrm{C} 26-\mathrm{H} 26 \mathrm{~B}$ & 0.9600 & $\mathrm{C} 78-\mathrm{H} 78$ & 0.9300 \\
\hline $\mathrm{C} 26-\mathrm{H} 26 \mathrm{C}$ & 0.9600 & $\mathrm{C} 79-\mathrm{C} 81$ & $1.492(4)$ \\
\hline $\mathrm{C} 27-\mathrm{H} 27 \mathrm{~A}$ & 0.9600 & $\mathrm{C} 80-\mathrm{H} 80 \mathrm{~A}$ & 0.9600 \\
\hline $\mathrm{C} 27-\mathrm{H} 27 \mathrm{~B}$ & 0.9600 & С $80-\mathrm{H} 80 \mathrm{~B}$ & 0.9600 \\
\hline $\mathrm{C} 27-\mathrm{H} 27 \mathrm{C}$ & 0.9600 & $\mathrm{C} 80-\mathrm{H} 80 \mathrm{C}$ & 0.9600 \\
\hline $\mathrm{C} 28-\mathrm{N} 4$ & $1.288(3)$ & C81-H81A & 0.9600 \\
\hline $\mathrm{C} 28-\mathrm{C} 37$ & $1.530(3)$ & C81-H81B & 0.9600 \\
\hline $\mathrm{N} 4-\mathrm{C} 29$ & $1.418(3)$ & $\mathrm{C} 81-\mathrm{H} 81 \mathrm{C}$ & 0.9600 \\
\hline $\mathrm{C} 29-\mathrm{C} 34$ & $1.396(4)$ & $\mathrm{O} 1-\mathrm{C} 82$ & $1.416(11)$ \\
\hline $\mathrm{C} 29-\mathrm{C} 30$ & $1.397(4)$ & $\mathrm{O} 1-\mathrm{C} 85$ & $1.436(12)$ \\
\hline $\mathrm{C} 30-\mathrm{C} 31$ & $1.388(4)$ & $\mathrm{C} 82-\mathrm{C} 83$ & $1.495(11)$ \\
\hline $\mathrm{C} 30-\mathrm{C} 35$ & $1.504(5)$ & $\mathrm{C} 82-\mathrm{H} 82 \mathrm{~A}$ & 0.9700 \\
\hline $\mathrm{C} 31-\mathrm{C} 32$ & $1.373(5)$ & $\mathrm{C} 82-\mathrm{H} 82 \mathrm{~B}$ & 0.9700 \\
\hline $\mathrm{C} 31-\mathrm{H} 31$ & 0.9300 & $\mathrm{C} 83-\mathrm{C} 84$ & $1.530(13)$ \\
\hline $\mathrm{C} 32-\mathrm{C} 33$ & $1.372(5)$ & $\mathrm{C} 83-\mathrm{H} 83 \mathrm{~A}$ & 0.9700 \\
\hline C $32-\mathrm{H} 32$ & 0.9300 & С $83-\mathrm{H} 83 \mathrm{~B}$ & 0.9700 \\
\hline $\mathrm{C} 33-\mathrm{C} 34$ & $1.397(4)$ & $\mathrm{C} 84-\mathrm{C} 85$ & $1.461(12)$ \\
\hline
\end{tabular}




\begin{tabular}{|c|c|c|c|}
\hline C $33-\mathrm{H} 33$ & 0.9300 & $\mathrm{C} 84-\mathrm{H} 84 \mathrm{~A}$ & 0.9700 \\
\hline $\mathrm{C} 34-\mathrm{C} 36$ & $1.494(4)$ & C84-H84B & 0.9700 \\
\hline C $35-\mathrm{H} 35 \mathrm{~A}$ & 0.9600 & $\mathrm{C} 85-\mathrm{H} 85 \mathrm{~A}$ & 0.9700 \\
\hline C35-H35B & 0.9600 & C85-H85B & 0.9700 \\
\hline $\mathrm{C} 35-\mathrm{H} 35 \mathrm{C}$ & 0.9600 & $\mathrm{O} 1^{\prime}-\mathrm{C} 82^{\prime}$ & $1.436(12)$ \\
\hline $\mathrm{C} 36-\mathrm{H} 36 \mathrm{~A}$ & 0.9600 & $\mathrm{O} 1^{\prime}-\mathrm{C} 85^{\prime}$ & $1.444(13)$ \\
\hline C36-H36B & 0.9600 & $\mathrm{C} 82^{\prime}-\mathrm{C} 83^{\prime}$ & $1.489(13)$ \\
\hline $\mathrm{C} 36-\mathrm{H} 36 \mathrm{C}$ & 0.9600 & $\mathrm{C} 82^{\prime}-\mathrm{H} 82 \mathrm{C}$ & 0.9700 \\
\hline $\mathrm{C} 37-\mathrm{N} 5$ & $1.261(3)$ & $\mathrm{C} 82^{\prime}-\mathrm{H} 82 \mathrm{D}$ & 0.9700 \\
\hline $\mathrm{C} 37-\mathrm{N} 6$ & $1.429(3)$ & $\mathrm{C} 83^{\prime}-\mathrm{C} 84^{\prime}$ & $1.508(14)$ \\
\hline $\mathrm{N} 5-\mathrm{C} 38$ & $1.412(3)$ & $\mathrm{C} 83^{\prime}-\mathrm{H} 83 \mathrm{C}$ & 0.9700 \\
\hline C $38-\mathrm{C} 39$ & $1.399(4)$ & $\mathrm{C} 83^{\prime}-\mathrm{H} 83 \mathrm{D}$ & 0.9700 \\
\hline $\mathrm{C} 38-\mathrm{C} 43$ & $1.399(4)$ & $\mathrm{C} 84^{\prime}-\mathrm{C} 85^{\prime}$ & $1.487(12)$ \\
\hline $\mathrm{C} 39-\mathrm{C} 40$ & $1.384(5)$ & $\mathrm{C} 84^{\prime}-\mathrm{H} 84 \mathrm{C}$ & 0.9700 \\
\hline $\mathrm{C} 39-\mathrm{C} 44$ & $1.498(5)$ & $\mathrm{C} 84^{\prime}-\mathrm{H} 84 \mathrm{D}$ & 0.9700 \\
\hline $\mathrm{C} 40-\mathrm{C} 41$ & $1.359(6)$ & $\mathrm{C} 85^{\prime}-\mathrm{H} 85 \mathrm{C}$ & 0.9700 \\
\hline $\mathrm{C} 40-\mathrm{H} 40$ & 0.9300 & $\mathrm{C} 85^{\prime}-\mathrm{H} 85 \mathrm{D}$ & 0.9700 \\
\hline $\mathrm{C} 41-\mathrm{C} 42$ & $1.378(6)$ & $\mathrm{O} 2-\mathrm{C} 86$ & $1.445(11)$ \\
\hline $\mathrm{C} 41-\mathrm{H} 41$ & 0.9300 & $\mathrm{O} 2-\mathrm{C} 89$ & $1.482(11)$ \\
\hline $\mathrm{C} 42-\mathrm{C} 43$ & $1.385(5)$ & $\mathrm{C} 86-\mathrm{C} 87$ & $1.510(12)$ \\
\hline $\mathrm{C} 42-\mathrm{H} 42$ & 0.9300 & $\mathrm{C} 86-\mathrm{H} 86 \mathrm{~A}$ & 0.9700 \\
\hline $\mathrm{C} 43-\mathrm{C} 45$ & $1.497(5)$ & C86-H86B & 0.9700 \\
\hline $\mathrm{C} 44-\mathrm{H} 44 \mathrm{~A}$ & 0.9600 & $\mathrm{C} 87-\mathrm{C} 88$ & $1.510(15)$ \\
\hline $\mathrm{C} 44-\mathrm{H} 44 \mathrm{~B}$ & 0.9600 & C87-H87A & 0.9700 \\
\hline $\mathrm{C} 44-\mathrm{H} 44 \mathrm{C}$ & 0.9600 & C87-H87B & 0.9700 \\
\hline $\mathrm{C} 45-\mathrm{H} 45 \mathrm{~A}$ & 0.9600 & $\mathrm{C} 88-\mathrm{C} 89$ & $1.511(12)$ \\
\hline $\mathrm{C} 45-\mathrm{H} 45 \mathrm{~B}$ & 0.9600 & $\mathrm{C} 88-\mathrm{H} 88 \mathrm{~A}$ & 0.9700 \\
\hline $\mathrm{C} 45-\mathrm{H} 45 \mathrm{C}$ & 0.9600 & $\mathrm{C} 88-\mathrm{H} 88 \mathrm{~B}$ & 0.9700 \\
\hline $\mathrm{C} 46-\mathrm{N} 6$ & $1.354(3)$ & C89-H89A & 0.9700 \\
\hline $\mathrm{C} 46-\mathrm{C} 55$ & $1.414(3)$ & C $89-\mathrm{H} 89 \mathrm{~B}$ & 0.9700 \\
\hline N6-C47 & $1.459(3)$ & $\mathrm{O} 2^{\prime}-\mathrm{C} 89^{\prime}$ & $1.469(13)$ \\
\hline $\mathrm{C} 47-\mathrm{C} 48$ & $1.389(4)$ & $\mathrm{O} 2^{\prime}-\mathrm{C} 86^{\prime}$ & $1.477(13)$ \\
\hline $\mathrm{C} 47-\mathrm{C} 52$ & $1.398(4)$ & $\mathrm{C} 86^{\prime}-\mathrm{C} 87^{\prime}$ & $1.539(13)$ \\
\hline $\mathrm{C} 48-\mathrm{C} 49$ & $1.388(4)$ & $\mathrm{C} 86^{\prime}-\mathrm{H} 86 \mathrm{C}$ & 0.9700 \\
\hline $\mathrm{C} 48-\mathrm{C} 53$ & $1.497(4)$ & $\mathrm{C} 86^{\prime}-\mathrm{H} 86 \mathrm{D}$ & 0.9700 \\
\hline $\mathrm{C} 49-\mathrm{C} 50$ & $1.366(6)$ & $\mathrm{C} 87^{\prime}-\mathrm{C} 88^{\prime}$ & $1.492(15)$ \\
\hline C49-H49 & 0.9300 & $\mathrm{C} 87^{\prime}-\mathrm{H} 87 \mathrm{C}$ & 0.9700 \\
\hline $\mathrm{C} 50-\mathrm{C} 51$ & $1.365(6)$ & $\mathrm{C} 87^{\prime}-\mathrm{H} 87 \mathrm{D}$ & 0.9700 \\
\hline $\mathrm{C} 50-\mathrm{H} 50$ & 0.9300 & $\mathrm{C} 88^{\prime}-\mathrm{C} 89^{\prime}$ & $1.515(14)$ \\
\hline $\mathrm{C} 51-\mathrm{C} 52$ & $1.395(4)$ & $\mathrm{C} 88^{\prime}-\mathrm{H} 88 \mathrm{C}$ & 0.9700 \\
\hline C51-H51 & 0.9300 & $\mathrm{C} 88^{\prime}-\mathrm{H} 88 \mathrm{D}$ & 0.9700 \\
\hline $\mathrm{C} 52-\mathrm{C} 54$ & $1.486(4)$ & $\mathrm{C} 89^{\prime}-\mathrm{H} 89 \mathrm{C}$ & 0.9700 \\
\hline $\mathrm{C} 53-\mathrm{H} 53 \mathrm{~A}$ & 0.9600 & $\mathrm{C} 89^{\prime}-\mathrm{H} 89 \mathrm{D}$ & 0.9700 \\
\hline C53-H53B & 0.9600 & & \\
\hline $\mathrm{C} 10-\mathrm{Fe} 1-\mathrm{C} 1$ & $84.67(10)$ & $\mathrm{C} 52-\mathrm{C} 54-\mathrm{H} 54 \mathrm{C}$ & 109.5 \\
\hline $\mathrm{C} 10-\mathrm{Fe} 1-\mathrm{C} 19$ & $94.05(11)$ & $\mathrm{H} 54 \mathrm{~A}-\mathrm{C} 54-\mathrm{H} 54 \mathrm{C}$ & 109.5 \\
\hline $\mathrm{C} 1-\mathrm{Fe} 1-\mathrm{C} 19$ & $177.81(11)$ & $\mathrm{H} 54 \mathrm{~B}-\mathrm{C} 54-\mathrm{H} 54 \mathrm{C}$ & 109.5 \\
\hline
\end{tabular}




\begin{tabular}{|c|c|}
\hline $\mathrm{C} 10-\mathrm{Fe} 1-\mathrm{C} 46$ & $171.45(10)$ \\
\hline $\mathrm{C} 1-\mathrm{Fe} 1-\mathrm{C} 46$ & $86.80(9)$ \\
\hline $\mathrm{C} 19-\mathrm{Fe} 1-\mathrm{C} 46$ & $94.46(10)$ \\
\hline $\mathrm{C} 10-\mathrm{Fe} 1-\mathrm{C} 73$ & $97.51(10)$ \\
\hline $\mathrm{C} 1-\mathrm{Fe} 1-\mathrm{C} 73$ & $88.02(9)$ \\
\hline $\mathrm{C} 19-\mathrm{Fe} 1-\mathrm{C} 73$ & $90.39(10)$ \\
\hline $\mathrm{C} 46-\mathrm{Fe} 1-\mathrm{C} 73$ & $81.56(9)$ \\
\hline $\mathrm{C} 10-\mathrm{Fe} 1-\mathrm{C} 28$ & $99.78(10)$ \\
\hline $\mathrm{C} 1-\mathrm{Fe} 1-\mathrm{C} 28$ & $96.36(10)$ \\
\hline $\mathrm{C} 19-\mathrm{Fe} 1-\mathrm{C} 28$ & $85.61(10)$ \\
\hline $\mathrm{C} 46-\mathrm{Fe} 1-\mathrm{C} 28$ & $81.76(10)$ \\
\hline $\mathrm{C} 73-\mathrm{Fe} 1-\mathrm{C} 28$ & $162.48(9)$ \\
\hline $\mathrm{N} 1^{\prime}-\mathrm{C} 1-\mathrm{Fe} 1$ & $175.6(11)$ \\
\hline $\mathrm{N} 1-\mathrm{C} 1-\mathrm{Fe} 1$ & $168.3(11)$ \\
\hline $\mathrm{C} 1-\mathrm{N} 1-\mathrm{C} 2$ & $172(2)$ \\
\hline $\mathrm{C} 3-\mathrm{C} 2-\mathrm{C} 7$ & $122.9(10)$ \\
\hline $\mathrm{C} 3-\mathrm{C} 2-\mathrm{N} 1$ & $118.3(13)$ \\
\hline $\mathrm{C} 7-\mathrm{C} 2-\mathrm{N} 1$ & $118.8(13)$ \\
\hline $\mathrm{C} 4-\mathrm{C} 3-\mathrm{C} 2$ & $116.8(11)$ \\
\hline $\mathrm{C} 4-\mathrm{C} 3-\mathrm{C} 8$ & $116.5(17)$ \\
\hline $\mathrm{C} 2-\mathrm{C} 3-\mathrm{C} 8$ & $126.7(18)$ \\
\hline $\mathrm{C} 5-\mathrm{C} 4-\mathrm{C} 3$ & $121.5(12)$ \\
\hline $\mathrm{C} 5-\mathrm{C} 4-\mathrm{H} 4$ & 119.2 \\
\hline $\mathrm{C} 3-\mathrm{C} 4-\mathrm{H} 4$ & 119.2 \\
\hline $\mathrm{C} 6-\mathrm{C} 5-\mathrm{C} 4$ & $120.3(12)$ \\
\hline $\mathrm{C} 6-\mathrm{C} 5-\mathrm{H} 5$ & 119.8 \\
\hline $\mathrm{C} 4-\mathrm{C} 5-\mathrm{H} 5$ & 119.8 \\
\hline $\mathrm{C} 5-\mathrm{C} 6-\mathrm{C} 7$ & $121.6(12)$ \\
\hline $\mathrm{C} 5-\mathrm{C} 6-\mathrm{H} 6$ & 119.2 \\
\hline $\mathrm{C} 7-\mathrm{C} 6-\mathrm{H} 6$ & 119.2 \\
\hline $\mathrm{C} 6-\mathrm{C} 7-\mathrm{C} 2$ & $116.8(11)$ \\
\hline $\mathrm{C} 6-\mathrm{C} 7-\mathrm{C} 9$ & $115.8(17)$ \\
\hline $\mathrm{C} 2-\mathrm{C} 7-\mathrm{C} 9$ & $127.2(16)$ \\
\hline $\mathrm{C} 3-\mathrm{C} 8-\mathrm{H} 8 \mathrm{~A}$ & 109.5 \\
\hline $\mathrm{C} 3-\mathrm{C} 8-\mathrm{H} 8 \mathrm{~B}$ & 109.5 \\
\hline $\mathrm{H} 8 \mathrm{~A}-\mathrm{C} 8-\mathrm{H} 8 \mathrm{~B}$ & 109.5 \\
\hline $\mathrm{C} 3-\mathrm{C} 8-\mathrm{H} 8 \mathrm{C}$ & 109.5 \\
\hline $\mathrm{H} 8 \mathrm{~A}-\mathrm{C} 8-\mathrm{H} 8 \mathrm{C}$ & 109.5 \\
\hline $\mathrm{H} 8 \mathrm{~B}-\mathrm{C} 8-\mathrm{H} 8 \mathrm{C}$ & 109.5 \\
\hline $\mathrm{C} 7-\mathrm{C} 9-\mathrm{H} 9 \mathrm{~A}$ & 109.5 \\
\hline $\mathrm{C} 7-\mathrm{C} 9-\mathrm{H} 9 \mathrm{~B}$ & 109.5 \\
\hline $\mathrm{H} 9 \mathrm{~A}-\mathrm{C} 9-\mathrm{H} 9 \mathrm{~B}$ & 109.5 \\
\hline $\mathrm{C} 7-\mathrm{C} 9-\mathrm{H} 9 \mathrm{C}$ & 109.5 \\
\hline $\mathrm{H} 9 \mathrm{~A}-\mathrm{C} 9-\mathrm{H} 9 \mathrm{C}$ & 109.5 \\
\hline $\mathrm{H} 9 \mathrm{~B}-\mathrm{C} 9-\mathrm{H} 9 \mathrm{C}$ & 109.5 \\
\hline $\mathrm{C} 1-\mathrm{N} 1^{\prime}-\mathrm{C} 2^{\prime}$ & $172.4(18)$ \\
\hline $\mathrm{C} 3^{\prime}-\mathrm{C} 2^{\prime}-\mathrm{C} 7^{\prime}$ & $122.8(9)$ \\
\hline $\mathrm{C} 3^{\prime}-\mathrm{C} 2^{\prime}-\mathrm{N} 1^{\prime}$ & $119.8(12)$ \\
\hline
\end{tabular}

$\begin{array}{ll}\mathrm{C} 64-\mathrm{C} 55-\mathrm{C} 46 & 111.4(2) \\ \mathrm{C} 64-\mathrm{C} 55-\mathrm{N} 7 & 122.4(2) \\ \mathrm{C} 46-\mathrm{C} 55-\mathrm{N} 7 & 126.2(2) \\ \mathrm{C} 56-\mathrm{N} 7-\mathrm{C} 55 & 127.5(2) \\ \mathrm{C} 56-\mathrm{N} 7-\mathrm{H} 7 & 118.2(17) \\ \mathrm{C} 55-\mathrm{N} 7-\mathrm{H} 7 & 114.2(17) \\ \mathrm{N} 7-\mathrm{C} 56-\mathrm{C} 61 & 122.5(3) \\ \mathrm{N} 7-\mathrm{C} 56-\mathrm{C} 57 & 116.8(3) \\ \mathrm{C} 61-\mathrm{C} 56-\mathrm{C} 57 & 120.7(3) \\ \mathrm{C} 58-\mathrm{C} 57-\mathrm{C} 56 & 118.2(4) \\ \mathrm{C} 58-\mathrm{C} 57-\mathrm{C} 62 & 120.5(3) \\ \mathrm{C} 56-\mathrm{C} 57-\mathrm{C} 62 & 121.3(3) \\ \mathrm{C} 59-\mathrm{C} 58-\mathrm{C} 57 & 121.5(4) \\ \mathrm{C} 59-\mathrm{C} 58-\mathrm{H} 58 & 119.3\end{array}$

$\mathrm{C} 59-\mathrm{C} 58-\mathrm{H} 58 \quad 119.3$

C57-C58-H58 119.3

$\mathrm{C} 58-\mathrm{C} 59-\mathrm{C} 60 \quad 119.8$ (4)

$\mathrm{C} 58-\mathrm{C} 59-\mathrm{H} 59 \quad 120.1$

C60-C59-H59 120.1

C59-C60-C61 121.8 (4)

$\mathrm{C} 59-\mathrm{C} 60-\mathrm{H} 60 \quad 119.1$

$\mathrm{C} 61-\mathrm{C} 60-\mathrm{H} 60 \quad 119.1$

C60-C61-C56 117.6 (3)

$\mathrm{C} 60-\mathrm{C} 61-\mathrm{C} 63 \quad 118.1$ (3)

C56-C61-C63 124.2 (3)

$\mathrm{C} 57-\mathrm{C} 62-\mathrm{H} 62 \mathrm{~A}$

$\mathrm{C} 57-\mathrm{C} 62-\mathrm{H} 62 \mathrm{~B} \quad 109.5$

$\mathrm{H} 62 \mathrm{~A}-\mathrm{C} 62-\mathrm{H} 62 \mathrm{~B} \quad 109.5$

$\mathrm{C} 57-\mathrm{C} 62-\mathrm{H} 62 \mathrm{C} \quad 109.5$

$\mathrm{H} 62 \mathrm{~A}-\mathrm{C} 62-\mathrm{H} 62 \mathrm{C} \quad 109.5$

H62B-C62-H62C 109.5

C61-C63-H63A 109.5

C61- C63-H63B $\quad 109.5$

$\mathrm{H} 63 \mathrm{~A}-\mathrm{C} 63-\mathrm{H} 63 \mathrm{~B} \quad 109.5$

C61- $663-\mathrm{H} 63 \mathrm{C} \quad 109.5$

$\mathrm{H} 63 \mathrm{~A}-\mathrm{C} 63-\mathrm{H} 63 \mathrm{C} \quad 109.5$

H63B-C63-H63C $\quad 109.5$

$\mathrm{N} 8$ - $\mathrm{C} 64-\mathrm{C} 55 \quad 125.1(10)$

$\mathrm{N} 8-\mathrm{C} 64-\mathrm{C} 55 \quad 132.9$ (7)

$\mathrm{N} 8{ }^{\prime}-\mathrm{C} 64-\mathrm{C} 73 \quad 119.1(9)$

$\mathrm{N} 8-\mathrm{C} 64-\mathrm{C} 73 \quad 111.8(7)$

$\mathrm{C} 55-\mathrm{C} 64-\mathrm{C} 73 \quad 115.3$ (2)

C64-N8-C65 129.4 (19)

$\mathrm{C} 64-\mathrm{N} 8-\mathrm{H} 8 \quad 115.3$

$\mathrm{C} 65-\mathrm{N} 8-\mathrm{H} 8 \quad 115.3$

C66- C65-C70 121.4 (9)

C66-C65-N8 $122.7(11)$

$\mathrm{C} 70-\mathrm{C} 65-\mathrm{N} 8 \quad 115.1(10)$

C65-C66-C67 119.8 (9) 


\begin{tabular}{|c|c|c|c|}
\hline $\mathrm{C} 7^{\prime}-\mathrm{C} 2^{\prime}-\mathrm{N} 1^{\prime}$ & $117.4(12)$ & $\mathrm{C} 65-\mathrm{C} 66-\mathrm{C} 71$ & $122.0(11)$ \\
\hline $\mathrm{C} 4^{\prime}-\mathrm{C} 3^{\prime}-\mathrm{C} 2^{\prime}$ & $116.8(11)$ & $\mathrm{C} 67-\mathrm{C} 66-\mathrm{C} 71$ & $118.0(11)$ \\
\hline $\mathrm{C} 4^{\prime}-\mathrm{C} 3^{\prime}-\mathrm{C} 8^{\prime}$ & $123.2(16)$ & C68-C67-C66 & $118.9(9)$ \\
\hline $\mathrm{C} 2^{\prime}-\mathrm{C} 3^{\prime}-\mathrm{C} 8^{\prime}$ & $119.9(16)$ & $\mathrm{C} 68-\mathrm{C} 67-\mathrm{H} 67$ & 120.5 \\
\hline $\mathrm{C} 5^{\prime}-\mathrm{C} 4^{\prime}-\mathrm{C} 3^{\prime}$ & $121.5(11)$ & C66-C67-H67 & 120.5 \\
\hline $\mathrm{C} 5^{\prime}-\mathrm{C} 4^{\prime}-\mathrm{H} 4^{\prime}$ & 119.2 & C67-C68-C69 & $121.2(9)$ \\
\hline $\mathrm{C} 3^{\prime}-\mathrm{C} 4^{\prime}-\mathrm{H} 4^{\prime}$ & 119.2 & C67-C68-H68 & 119.4 \\
\hline $\mathrm{C} 6^{\prime}-\mathrm{C} 5^{\prime}-\mathrm{C} 4^{\prime}$ & $119.9(10)$ & $\mathrm{C} 69-\mathrm{C} 68-\mathrm{H} 68$ & 119.4 \\
\hline $\mathrm{C} 6^{\prime}-\mathrm{C} 5^{\prime}-\mathrm{H} 5^{\prime}$ & 120.1 & $\mathrm{C} 68-\mathrm{C} 69-\mathrm{C} 70$ & $121.1(9)$ \\
\hline $\mathrm{C} 4^{\prime}-\mathrm{C} 5^{\prime}-\mathrm{H} 5^{\prime}$ & 120.1 & $\mathrm{C} 68-\mathrm{C} 69-\mathrm{H} 69$ & 119.5 \\
\hline $\mathrm{C} 5^{\prime}-\mathrm{C} 6^{\prime}-\mathrm{C} 7^{\prime}$ & $122.0(11)$ & $\mathrm{C} 70-\mathrm{C} 69-\mathrm{H} 69$ & 119.5 \\
\hline $\mathrm{C} 5^{\prime}-\mathrm{C} 6^{\prime}-\mathrm{H} 6^{\prime}$ & 119.0 & $\mathrm{C} 69-\mathrm{C} 70-\mathrm{C} 65$ & $117.2(9)$ \\
\hline $\mathrm{C} 7^{\prime}-\mathrm{C} 6^{\prime}-\mathrm{H} 6^{\prime}$ & 119.0 & $\mathrm{C} 69-\mathrm{C} 70-\mathrm{C} 72$ & $119.4(11)$ \\
\hline $\mathrm{C} 6^{\prime}-\mathrm{C} 7^{\prime}-\mathrm{C} 2^{\prime}$ & $116.9(10)$ & $\mathrm{C} 65-\mathrm{C} 70-\mathrm{C} 72$ & $123.3(11)$ \\
\hline $\mathrm{C} 6^{\prime}-\mathrm{C} 7^{\prime}-\mathrm{C} 9^{\prime}$ & $125.3(16)$ & $\mathrm{C} 66-\mathrm{C} 71-\mathrm{H} 71 \mathrm{~A}$ & 109.5 \\
\hline $\mathrm{C} 2^{\prime}-\mathrm{C} 7^{\prime}-\mathrm{C} 9^{\prime}$ & $117.6(14)$ & $\mathrm{C} 66-\mathrm{C} 71-\mathrm{H} 71 \mathrm{~B}$ & 109.5 \\
\hline $\mathrm{C}^{\prime}-\mathrm{C} 8^{\prime}-\mathrm{H} 8 \mathrm{D}$ & 109.5 & $\mathrm{H} 71 \mathrm{~A}-\mathrm{C} 71-\mathrm{H} 71 \mathrm{~B}$ & 109.5 \\
\hline $\mathrm{C} 3^{\prime}-\mathrm{C} 8^{\prime}-\mathrm{H} 8 \mathrm{E}$ & 109.5 & $\mathrm{C} 66-\mathrm{C} 71-\mathrm{H} 71 \mathrm{C}$ & 109.5 \\
\hline $\mathrm{H} 8 \mathrm{D}-\mathrm{C} 8^{\prime}-\mathrm{H} 8 \mathrm{E}$ & 109.5 & $\mathrm{H} 71 \mathrm{~A}-\mathrm{C} 71-\mathrm{H} 71 \mathrm{C}$ & 109.5 \\
\hline $\mathrm{C} 3^{\prime}-\mathrm{C} 8^{\prime}-\mathrm{H} 8 \mathrm{~F}$ & 109.5 & $\mathrm{H} 71 \mathrm{~B}-\mathrm{C} 71-\mathrm{H} 71 \mathrm{C}$ & 109.5 \\
\hline $\mathrm{H} 8 \mathrm{D}-\mathrm{C} 8^{\prime}-\mathrm{H} 8 \mathrm{~F}$ & 109.5 & $\mathrm{C} 70-\mathrm{C} 72-\mathrm{H} 72 \mathrm{~A}$ & 109.5 \\
\hline $\mathrm{H} 8 \mathrm{E}-\mathrm{C} 8^{\prime}-\mathrm{H} 8 \mathrm{~F}$ & 109.5 & $\mathrm{C} 70-\mathrm{C} 72-\mathrm{H} 72 \mathrm{~B}$ & 109.5 \\
\hline $\mathrm{C}^{\prime}-\mathrm{C} 9^{\prime}-\mathrm{H} 9 \mathrm{D}$ & 109.5 & $\mathrm{H} 72 \mathrm{~A}-\mathrm{C} 72-\mathrm{H} 72 \mathrm{~B}$ & 109.5 \\
\hline $\mathrm{C} 7^{\prime}-\mathrm{C} 9^{\prime}-\mathrm{H} 9 \mathrm{E}$ & 109.5 & $\mathrm{C} 70-\mathrm{C} 72-\mathrm{H} 72 \mathrm{C}$ & 109.5 \\
\hline $\mathrm{H} 9 \mathrm{D}-\mathrm{C} 9^{\prime}-\mathrm{H} 9 \mathrm{E}$ & 109.5 & $\mathrm{H} 72 \mathrm{~A}-\mathrm{C} 72-\mathrm{H} 72 \mathrm{C}$ & 109.5 \\
\hline $\mathrm{C} 7^{\prime}-\mathrm{C} 9^{\prime}-\mathrm{H} 9 \mathrm{~F}$ & 109.5 & $\mathrm{H} 72 \mathrm{~B}-\mathrm{C} 72-\mathrm{H} 72 \mathrm{C}$ & 109.5 \\
\hline $\mathrm{H} 9 \mathrm{D}-\mathrm{C}^{\prime}-\mathrm{H} 9 \mathrm{~F}$ & 109.5 & $\mathrm{C} 64-\mathrm{N} 8^{\prime}-\mathrm{C} 65^{\prime}$ & $136(2)$ \\
\hline $\mathrm{H} 9 \mathrm{E}-\mathrm{C} 9^{\prime}-\mathrm{H} 9 \mathrm{~F}$ & 109.5 & $\mathrm{C} 64-\mathrm{N} 8^{\prime}-\mathrm{H} 8^{\prime}$ & 112.1 \\
\hline $\mathrm{N} 2-\mathrm{C} 10-\mathrm{Fe} 1$ & $170.3(2)$ & $\mathrm{C} 65^{\prime}-\mathrm{N} 8^{\prime}-\mathrm{H} 8^{\prime}$ & 112.1 \\
\hline $\mathrm{C} 10-\mathrm{N} 2-\mathrm{C} 11$ & $177.8(3)$ & $\mathrm{C} 66^{\prime}-\mathrm{C} 65^{\prime}-\mathrm{C} 70^{\prime}$ & $124.6(12)$ \\
\hline $\mathrm{C} 16-\mathrm{C} 11-\mathrm{N} 2$ & $119.7(3)$ & $\mathrm{C} 66^{\prime}-\mathrm{C} 65^{\prime}-\mathrm{N} 8^{\prime}$ & $120.4(13)$ \\
\hline $\mathrm{C} 16-\mathrm{C} 11-\mathrm{C} 12$ & $124.2(3)$ & $\mathrm{C} 70^{\prime}-\mathrm{C} 65^{\prime}-\mathrm{N} 8^{\prime}$ & $115.0(13)$ \\
\hline $\mathrm{N} 2-\mathrm{C} 11-\mathrm{C} 12$ & $116.1(3)$ & $\mathrm{C} 65^{\prime}-\mathrm{C} 66^{\prime}-\mathrm{C} 67^{\prime}$ & $116.1(12)$ \\
\hline $\mathrm{C} 13-\mathrm{C} 12-\mathrm{C} 11$ & $116.0(4)$ & $\mathrm{C} 65^{\prime}-\mathrm{C} 66^{\prime}-\mathrm{C} 71^{\prime}$ & $126.0(14)$ \\
\hline $\mathrm{C} 13-\mathrm{C} 12-\mathrm{C} 17$ & $122.7(4)$ & $\mathrm{C} 67^{\prime}-\mathrm{C} 66^{\prime}-\mathrm{C} 71^{\prime}$ & $117.9(14)$ \\
\hline $\mathrm{C} 11-\mathrm{C} 12-\mathrm{C} 17$ & $121.2(3)$ & $\mathrm{C} 68^{\prime}-\mathrm{C} 67^{\prime}-\mathrm{C} 66^{\prime}$ & $120.5(14)$ \\
\hline $\mathrm{C} 14-\mathrm{C} 13-\mathrm{C} 12$ & $121.9(4)$ & $\mathrm{C} 68^{\prime}-\mathrm{C} 67^{\prime}-\mathrm{H} 67^{\prime}$ & 119.7 \\
\hline $\mathrm{C} 14-\mathrm{C} 13-\mathrm{H} 13$ & 119.0 & $\mathrm{C} 66^{\prime}-\mathrm{C} 67^{\prime}-\mathrm{H} 67^{\prime}$ & 119.7 \\
\hline $\mathrm{C} 12-\mathrm{C} 13-\mathrm{H} 13$ & 119.0 & $\mathrm{C} 67^{\prime}-\mathrm{C} 68^{\prime}-\mathrm{C} 69^{\prime}$ & $123.1(13)$ \\
\hline $\mathrm{C} 13-\mathrm{C} 14-\mathrm{C} 15$ & $122.4(4)$ & $\mathrm{C} 67^{\prime}-\mathrm{C} 68^{\prime}-\mathrm{H} 68^{\prime}$ & 118.5 \\
\hline $\mathrm{C} 13-\mathrm{C} 14-\mathrm{H} 14$ & 118.8 & $\mathrm{C} 69^{\prime}-\mathrm{C} 68^{\prime}-\mathrm{H} 68^{\prime}$ & 118.5 \\
\hline $\mathrm{C} 15-\mathrm{C} 14-\mathrm{H} 14$ & 118.8 & $\mathrm{C} 68^{\prime}-\mathrm{C} 69^{\prime}-\mathrm{C} 70^{\prime}$ & $118.0(11)$ \\
\hline $\mathrm{C} 14-\mathrm{C} 15-\mathrm{C} 16$ & $118.9(4)$ & $\mathrm{C} 68^{\prime}-\mathrm{C} 69^{\prime}-\mathrm{H} 69^{\prime}$ & 121.0 \\
\hline $\mathrm{C} 14-\mathrm{C} 15-\mathrm{H} 15$ & 120.5 & $\mathrm{C} 70^{\prime}-\mathrm{C} 69^{\prime}-\mathrm{H} 69^{\prime}$ & 121.0 \\
\hline $\mathrm{C} 16-\mathrm{C} 15-\mathrm{H} 15$ & 120.5 & $\mathrm{C} 69^{\prime}-\mathrm{C} 70^{\prime}-\mathrm{C} 65^{\prime}$ & $117.4(13)$ \\
\hline $\mathrm{C} 11-\mathrm{C} 16-\mathrm{C} 15$ & $116.5(4)$ & $\mathrm{C} 69^{\prime}-\mathrm{C} 70^{\prime}-\mathrm{C} 72^{\prime}$ & $122.6(15)$ \\
\hline $\mathrm{C} 11-\mathrm{C} 16-\mathrm{C} 18$ & $122.7(3)$ & $\mathrm{C} 65^{\prime}-\mathrm{C} 70^{\prime}-\mathrm{C} 72^{\prime}$ & $119.7(16)$ \\
\hline $\mathrm{C} 15-\mathrm{C} 16-\mathrm{C} 18$ & $120.8(4)$ & $\mathrm{C} 66^{\prime}-\mathrm{C} 71^{\prime}-\mathrm{H} 71 \mathrm{D}$ & 109.5 \\
\hline
\end{tabular}


C12-C17-H17A

C12-C17-H17B

H17A-C17-H17B

C12-C17- $\mathrm{H} 17 \mathrm{C}$

$\mathrm{H} 17 \mathrm{~A}-\mathrm{C} 17-\mathrm{H} 17 \mathrm{C}$

$\mathrm{H} 17 \mathrm{~B}-\mathrm{C} 17-\mathrm{H} 17 \mathrm{C}$

C16-C18-H18A

C16-C18-H18B

$\mathrm{H} 18 \mathrm{~A}-\mathrm{C} 18-\mathrm{H} 18 \mathrm{~B}$

C16- $18-\mathrm{H} 18 \mathrm{C}$

$\mathrm{H} 18 \mathrm{~A}-\mathrm{C} 18-\mathrm{H} 18 \mathrm{C}$

$\mathrm{H} 18 \mathrm{~B}-\mathrm{C} 18-\mathrm{H} 18 \mathrm{C}$

N3-C19-Fe1

$\mathrm{C} 19-\mathrm{N} 3-\mathrm{C} 20$

$\mathrm{C} 25-\mathrm{C} 20-\mathrm{C} 21$

$\mathrm{C} 25-\mathrm{C} 20-\mathrm{N} 3$

$\mathrm{C} 21-\mathrm{C} 20-\mathrm{N} 3$

$\mathrm{C} 22-\mathrm{C} 21-\mathrm{C} 20$

$\mathrm{C} 22-\mathrm{C} 21-\mathrm{C} 26$

$\mathrm{C} 20-\mathrm{C} 21-\mathrm{C} 26$

$\mathrm{C} 23-\mathrm{C} 22-\mathrm{C} 21$

$\mathrm{C} 23-\mathrm{C} 22-\mathrm{H} 22$

$\mathrm{C} 21-\mathrm{C} 22-\mathrm{H} 22$

$\mathrm{C} 22-\mathrm{C} 23-\mathrm{C} 24$

$\mathrm{C} 22-\mathrm{C} 23-\mathrm{H} 23$

$\mathrm{C} 24-\mathrm{C} 23-\mathrm{H} 23$

$\mathrm{C} 23-\mathrm{C} 24-\mathrm{C} 25$

$\mathrm{C} 23-\mathrm{C} 24-\mathrm{H} 24$

$\mathrm{C} 25-\mathrm{C} 24-\mathrm{H} 24$

$\mathrm{C} 20-\mathrm{C} 25-\mathrm{C} 24$

$\mathrm{C} 20-\mathrm{C} 25-\mathrm{C} 27$

$\mathrm{C} 24-\mathrm{C} 25-\mathrm{C} 27$

$\mathrm{C} 21-\mathrm{C} 26-\mathrm{H} 26 \mathrm{~A}$

$\mathrm{C} 21-\mathrm{C} 26-\mathrm{H} 26 \mathrm{~B}$

$\mathrm{H} 26 \mathrm{~A}-\mathrm{C} 26-\mathrm{H} 26 \mathrm{~B}$

$\mathrm{C} 21-\mathrm{C} 26-\mathrm{H} 26 \mathrm{C}$

$\mathrm{H} 26 \mathrm{~A}-\mathrm{C} 26-\mathrm{H} 26 \mathrm{C}$

$\mathrm{H} 26 \mathrm{~B}-\mathrm{C} 26-\mathrm{H} 26 \mathrm{C}$

$\mathrm{C} 25-\mathrm{C} 27-\mathrm{H} 27 \mathrm{~A}$

$\mathrm{C} 25-\mathrm{C} 27-\mathrm{H} 27 \mathrm{~B}$

$\mathrm{H} 27 \mathrm{~A}-\mathrm{C} 27-\mathrm{H} 27 \mathrm{~B}$

$\mathrm{C} 25-\mathrm{C} 27-\mathrm{H} 27 \mathrm{C}$

$\mathrm{H} 27 \mathrm{~A}-\mathrm{C} 27-\mathrm{H} 27 \mathrm{C}$

$\mathrm{H} 27 \mathrm{~B}-\mathrm{C} 27-\mathrm{H} 27 \mathrm{C}$

$\mathrm{N} 4-\mathrm{C} 28-\mathrm{C} 37$

$\mathrm{N} 4-\mathrm{C} 28-\mathrm{Fe} 1$

$\mathrm{C} 37-\mathrm{C} 28-\mathrm{Fe} 1$

$\mathrm{C} 28-\mathrm{N} 4-\mathrm{C} 29$
109.5

109.5

109.5

109.5

109.5

109.5

109.5

109.5

109.5

109.5

109.5

109.5

178.3 (2)

$174.2(3)$

$122.9(3)$

$119.1(3)$

$118.0(3)$

$117.1(4)$

$121.3(4)$

$121.6(3)$

120.9 (5)

119.6

119.6

$123.1(5)$

118.5

118.5

$119.1(5)$

120.4

120.4

117.0 (4)

$121.1(3)$

$122.0(4)$

109.5

109.5

109.5

109.5

109.5

109.5

109.5

109.5

109.5

109.5

109.5

109.5

109.9 (2)

$137.80(19)$

111.97 (16)

125.0 (2)
C66'- $771^{\prime}-\mathrm{H} 71 \mathrm{E}$

H71D-C71'-H71E

C66'-C $71^{\prime}-\mathrm{H} 71 \mathrm{~F}$

$\mathrm{H} 71 \mathrm{D}-\mathrm{C} 71^{\prime}-\mathrm{H} 71 \mathrm{~F}$

$\mathrm{H} 71 \mathrm{E}-\mathrm{C} 71^{\prime}-\mathrm{H} 71 \mathrm{~F}$

$\mathrm{C} 70^{\prime}-\mathrm{C} 72^{\prime}-\mathrm{H} 72 \mathrm{D}$

$\mathrm{C} 70^{\prime}-\mathrm{C} 72^{\prime}-\mathrm{H} 72 \mathrm{E}$

$\mathrm{H} 72 \mathrm{D}-\mathrm{C} 72^{\prime}-\mathrm{H} 72 \mathrm{E}$

$\mathrm{C} 70^{\prime}-\mathrm{C} 72^{\prime}-\mathrm{H} 72 \mathrm{~F}$

$\mathrm{H} 72 \mathrm{D}-\mathrm{C} 72^{\prime}-\mathrm{H} 72 \mathrm{~F}$

H72E-C $72^{\prime}-\mathrm{H} 72 \mathrm{~F}$

N9-C73-C64

$\mathrm{N} 9-\mathrm{C} 73-\mathrm{Fe} 1$

$\mathrm{C} 64-\mathrm{C} 73-\mathrm{Fe} 1$

C73-N9-C74

$\mathrm{C} 75-\mathrm{C} 74-\mathrm{C} 79$

C75-C74-N9

C79-C74-N9

$\mathrm{C} 74-\mathrm{C} 75-\mathrm{C} 76$

$\mathrm{C} 74-\mathrm{C} 75-\mathrm{C} 80$

C76-C75-C 80

C77-C76-C75

C77-C76-H76

C75-C76- $\mathrm{H} 76$

C78-C77-C76

C78-C77-H77

C76- C77-H77

C77-C78-C79

C77-C78-H78

C79-C78-H78

$\mathrm{C} 78-\mathrm{C} 79-\mathrm{C} 74$

$\mathrm{C} 78-\mathrm{C} 79-\mathrm{C} 81$

C74-C79-C81

C75-C $80-\mathrm{H} 80 \mathrm{~A}$

$\mathrm{C} 75-\mathrm{C} 80-\mathrm{H} 80 \mathrm{~B}$

$\mathrm{H} 80 \mathrm{~A}-\mathrm{C} 80-\mathrm{H} 80 \mathrm{~B}$

C75-C $80-\mathrm{H} 80 \mathrm{C}$

$\mathrm{H} 80 \mathrm{~A}-\mathrm{C} 80-\mathrm{H} 80 \mathrm{C}$

$\mathrm{H} 80 \mathrm{~B}-\mathrm{C} 80-\mathrm{H} 80 \mathrm{C}$

C79-C $81-\mathrm{H} 81 \mathrm{~A}$

C79-C $81-\mathrm{H} 81 \mathrm{~B}$

H81A-C $81-\mathrm{H} 81 \mathrm{~B}$

C79-C $81-\mathrm{H} 81 \mathrm{C}$

$\mathrm{H} 81 \mathrm{~A}-\mathrm{C} 81-\mathrm{H} 81 \mathrm{C}$

$\mathrm{H} 81 \mathrm{~B}-\mathrm{C} 81-\mathrm{H} 81 \mathrm{C}$

$\mathrm{C} 82-\mathrm{O} 1-\mathrm{C} 85$

$\mathrm{O} 1-\mathrm{C} 82-\mathrm{C} 83$

$\mathrm{O} 1-\mathrm{C} 82-\mathrm{H} 82 \mathrm{~A}$
109.5

109.5

109.5

109.5

109.5

109.5

109.5

109.5

109.5

109.5

109.5

$110.2(2)$

140.42 (18)

$109.28(15)$

128.2 (2)

120.4 (2)

117.8 (2)

120.7 (2)

118.4 (3)

121.3 (3)

120.3 (3)

$121.3(3)$

119.4

119.4

119.9 (3)

120.0

120.0

121.3 (3)

119.4

119.4

118.7 (3)

120.5 (3)

120.7 (2)

109.5

109.5

109.5

109.5

109.5

109.5

109.5

109.5

109.5

109.5

109.5

109.5

110.7 (14)

104.8 (12)

110.8 


\begin{tabular}{|c|c|}
\hline $\mathrm{C} 34-\mathrm{C} 29-\mathrm{C} 30$ & $120.6(3)$ \\
\hline $\mathrm{C} 34-\mathrm{C} 29-\mathrm{N} 4$ & $119.9(3)$ \\
\hline $\mathrm{C} 30-\mathrm{C} 29-\mathrm{N} 4$ & $118.8(3)$ \\
\hline $\mathrm{C} 31-\mathrm{C} 30-\mathrm{C} 29$ & $118.6(3)$ \\
\hline $\mathrm{C} 31-\mathrm{C} 30-\mathrm{C} 35$ & $120.2(3)$ \\
\hline $\mathrm{C} 29-\mathrm{C} 30-\mathrm{C} 35$ & $121.1(3)$ \\
\hline $\mathrm{C} 32-\mathrm{C} 31-\mathrm{C} 30$ & $121.3(3)$ \\
\hline $\mathrm{C} 32-\mathrm{C} 31-\mathrm{H} 31$ & 119.3 \\
\hline $\mathrm{C} 30-\mathrm{C} 31-\mathrm{H} 31$ & 119.3 \\
\hline $\mathrm{C} 33-\mathrm{C} 32-\mathrm{C} 31$ & $119.8(3)$ \\
\hline $\mathrm{C} 33-\mathrm{C} 32-\mathrm{H} 32$ & 120.1 \\
\hline $\mathrm{C} 31-\mathrm{C} 32-\mathrm{H} 32$ & 120.1 \\
\hline $\mathrm{C} 32-\mathrm{C} 33-\mathrm{C} 34$ & 121.0 \\
\hline $\mathrm{C} 32-\mathrm{C} 33-\mathrm{H} 33$ & 119.5 \\
\hline $\mathrm{C} 34-\mathrm{C} 33-\mathrm{H} 33$ & 119.5 \\
\hline $\mathrm{C} 29-\mathrm{C} 34-\mathrm{C} 33$ & $118.6(3)$ \\
\hline $\mathrm{C} 29-\mathrm{C} 34-\mathrm{C} 36$ & $120.7(3)$ \\
\hline $\mathrm{C} 33-\mathrm{C} 34-\mathrm{C} 36$ & $120.7(3)$ \\
\hline $\mathrm{C} 30-\mathrm{C} 35-\mathrm{H} 35 \mathrm{~A}$ & 109.5 \\
\hline $\mathrm{C} 30-\mathrm{C} 35-\mathrm{H} 35 \mathrm{~B}$ & 109.5 \\
\hline $\mathrm{H} 35 \mathrm{~A}-\mathrm{C} 35-\mathrm{H} 35 \mathrm{~B}$ & 109.5 \\
\hline $\mathrm{C} 30-\mathrm{C} 35-\mathrm{H} 35 \mathrm{C}$ & 109.5 \\
\hline $\mathrm{H} 35 \mathrm{~A}-\mathrm{C} 35-\mathrm{H} 35 \mathrm{C}$ & 109.5 \\
\hline $\mathrm{H} 35 \mathrm{~B}-\mathrm{C} 35-\mathrm{H} 35 \mathrm{C}$ & 109.5 \\
\hline $\mathrm{C} 34-\mathrm{C} 36-\mathrm{H} 36 \mathrm{~A}$ & 109.5 \\
\hline $\mathrm{C} 34-\mathrm{C} 36-\mathrm{H} 36 \mathrm{~B}$ & 109.5 \\
\hline $\mathrm{H} 36 \mathrm{~A}-\mathrm{C} 36-\mathrm{H} 36 \mathrm{~B}$ & 109.5 \\
\hline $\mathrm{C} 34-\mathrm{C} 36-\mathrm{H} 36 \mathrm{C}$ & 109.5 \\
\hline $\mathrm{H} 36 \mathrm{~A}-\mathrm{C} 36-\mathrm{H} 36 \mathrm{C}$ & 109.5 \\
\hline $\mathrm{H} 36 \mathrm{~B}-\mathrm{C} 36-\mathrm{H} 36 \mathrm{C}$ & 109.5 \\
\hline $\mathrm{N} 5-\mathrm{C} 37-\mathrm{N} 6$ & $116.3(2)$ \\
\hline $\mathrm{N} 5-\mathrm{C} 37-\mathrm{C} 28$ & $132.5(2)$ \\
\hline $\mathrm{N} 6-\mathrm{C} 37-\mathrm{C} 28$ & $111.2(2)$ \\
\hline $\mathrm{C} 37-\mathrm{N} 5-\mathrm{C} 38$ & $127.4(2)$ \\
\hline $\mathrm{C} 39-\mathrm{C} 38-\mathrm{C} 43$ & $120.4(3)$ \\
\hline $\mathrm{C} 39-\mathrm{C} 38-\mathrm{N} 5$ & $119.8(3)$ \\
\hline $\mathrm{C} 43-\mathrm{C} 38-\mathrm{N} 5$ & $118.9(3)$ \\
\hline $\mathrm{C} 40-\mathrm{C} 39-\mathrm{C} 38$ & $118.7(3)$ \\
\hline $\mathrm{C} 40-\mathrm{C} 39-\mathrm{C} 44$ & $121.1(3)$ \\
\hline $\mathrm{C} 38-\mathrm{C} 39-\mathrm{C} 44$ & $120.3(3)$ \\
\hline $\mathrm{C} 41-\mathrm{C} 40-\mathrm{C} 39$ & $121.4(4)$ \\
\hline $\mathrm{C} 41-\mathrm{C} 40-\mathrm{H} 40$ & 119.3 \\
\hline $\mathrm{C} 39-\mathrm{C} 40-\mathrm{H} 40$ & 119.3 \\
\hline $\mathrm{C} 40-\mathrm{C} 41-\mathrm{C} 42$ & $120.0(4)$ \\
\hline $\mathrm{C} 40-\mathrm{C} 41-\mathrm{H} 41$ & 120.0 \\
\hline $\mathrm{C} 42-\mathrm{C} 41-\mathrm{H} 41$ & 120.0 \\
\hline $\mathrm{C} 41-\mathrm{C} 42-\mathrm{C} 43$ & $121.0(4)$ \\
\hline$C 41 \quad C 42$ & \\
\hline
\end{tabular}

\begin{tabular}{|c|c|}
\hline $\mathrm{C} 83-\mathrm{C} 82-\mathrm{H} 82 \mathrm{~A}$ & 110.8 \\
\hline $\mathrm{O} 1-\mathrm{C} 82-\mathrm{H} 82 \mathrm{~B}$ & 110.8 \\
\hline $\mathrm{C} 83-\mathrm{C} 82-\mathrm{H} 82 \mathrm{~B}$ & 110.8 \\
\hline $\mathrm{H} 82 \mathrm{~A}-\mathrm{C} 82-\mathrm{H} 82 \mathrm{~B}$ & 108.9 \\
\hline $\mathrm{C} 82-\mathrm{C} 83-\mathrm{C} 84$ & $107.1(10)$ \\
\hline $\mathrm{C} 82-\mathrm{C} 83-\mathrm{H} 83 \mathrm{~A}$ & 110.3 \\
\hline $\mathrm{C} 84-\mathrm{C} 83-\mathrm{H} 83 \mathrm{~A}$ & 110.3 \\
\hline $\mathrm{C} 82-\mathrm{C} 83-\mathrm{H} 83 \mathrm{~B}$ & 110.3 \\
\hline $\mathrm{C} 84-\mathrm{C} 83-\mathrm{H} 83 \mathrm{~B}$ & 110.3 \\
\hline $\mathrm{H} 83 \mathrm{~A}-\mathrm{C} 83-\mathrm{H} 83 \mathrm{~B}$ & 108.5 \\
\hline $\mathrm{C} 85-\mathrm{C} 84-\mathrm{C} 83$ & $103.3(11)$ \\
\hline $\mathrm{C} 85-\mathrm{C} 84-\mathrm{H} 84 \mathrm{~A}$ & 111.1 \\
\hline $\mathrm{C} 83-\mathrm{C} 84-\mathrm{H} 84 \mathrm{~A}$ & 111.1 \\
\hline $\mathrm{C} 85-\mathrm{C} 84-\mathrm{H} 84 \mathrm{~B}$ & 111.1 \\
\hline $\mathrm{C} 83-\mathrm{C} 84-\mathrm{H} 84 \mathrm{~B}$ & 111.1 \\
\hline $\mathrm{H} 84 \mathrm{~A}-\mathrm{C} 84-\mathrm{H} 84 \mathrm{~B}$ & 109.1 \\
\hline $\mathrm{O} 1-\mathrm{C} 85-\mathrm{C} 84$ & $105.8(11)$ \\
\hline $\mathrm{O} 1-\mathrm{C} 85-\mathrm{H} 85 \mathrm{~A}$ & 110.6 \\
\hline $\mathrm{C} 84-\mathrm{C} 85-\mathrm{H} 85 \mathrm{~A}$ & 110.6 \\
\hline $\mathrm{O} 1-\mathrm{C} 85-\mathrm{H} 85 \mathrm{~B}$ & 110.6 \\
\hline $\mathrm{C} 84-\mathrm{C} 85-\mathrm{H} 85 \mathrm{~B}$ & 110.6 \\
\hline $\mathrm{H} 85 \mathrm{~A}-\mathrm{C} 85-\mathrm{H} 85 \mathrm{~B}$ & 108.7 \\
\hline $\mathrm{C} 82^{\prime}-\mathrm{O} 1^{\prime}-\mathrm{C} 85^{\prime}$ & $111.9(15)$ \\
\hline $\mathrm{O} 1^{\prime}-\mathrm{C} 82^{\prime}-\mathrm{C} 83^{\prime}$ & $100.4(14)$ \\
\hline $\mathrm{O} 1^{\prime}-\mathrm{C} 82^{\prime}-\mathrm{H} 82 \mathrm{C}$ & 111.7 \\
\hline $\mathrm{C} 83^{\prime}-\mathrm{C} 82^{\prime}-\mathrm{H} 82 \mathrm{C}$ & 111.7 \\
\hline $\mathrm{O} 1^{\prime}-\mathrm{C} 82^{\prime}-\mathrm{H} 82 \mathrm{D}$ & 111.7 \\
\hline $\mathrm{C} 83^{\prime}-\mathrm{C} 82^{\prime}-\mathrm{H} 82 \mathrm{D}$ & 111.7 \\
\hline $\mathrm{H} 82 \mathrm{C}-\mathrm{C} 82^{\prime}-\mathrm{H} 82 \mathrm{D}$ & 109.5 \\
\hline $\mathrm{C} 82^{\prime}-\mathrm{C} 83^{\prime}-\mathrm{C} 84^{\prime}$ & 103.5 \\
\hline $\mathrm{C} 82^{\prime}-\mathrm{C} 83^{\prime}-\mathrm{H} 83 \mathrm{C}$ & 111.1 \\
\hline $\mathrm{C} 84^{\prime}-\mathrm{C} 83^{\prime}-\mathrm{H} 83 \mathrm{C}$ & 111.1 \\
\hline $\mathrm{C} 82^{\prime}-\mathrm{C} 83^{\prime}-\mathrm{H} 83 \mathrm{D}$ & 111.1 \\
\hline $\mathrm{C} 84^{\prime}-\mathrm{C} 83^{\prime}-\mathrm{H} 83 \mathrm{D}$ & 111.1 \\
\hline $\mathrm{H} 83 \mathrm{C}-\mathrm{C} 83^{\prime}-\mathrm{H} 83 \mathrm{D}$ & 109.0 \\
\hline $\mathrm{C} 85^{\prime}-\mathrm{C} 84^{\prime}-\mathrm{C} 83^{\prime}$ & 107.0 \\
\hline $\mathrm{C} 85^{\prime}-\mathrm{C} 84^{\prime}-\mathrm{H} 84 \mathrm{C}$ & 110.3 \\
\hline $\mathrm{C} 83^{\prime}-\mathrm{C} 84^{\prime}-\mathrm{H} 84 \mathrm{C}$ & 110.3 \\
\hline $\mathrm{C} 85^{\prime}-\mathrm{C} 84^{\prime}-\mathrm{H} 84 \mathrm{D}$ & 110.3 \\
\hline $\mathrm{C} 83^{\prime}-\mathrm{C} 84^{\prime}-\mathrm{H} 84 \mathrm{D}$ & 110.3 \\
\hline $\mathrm{H} 84 \mathrm{C}-\mathrm{C} 84^{\prime}-\mathrm{H} 84 \mathrm{D}$ & 108.6 \\
\hline $\mathrm{O} 1^{\prime}-\mathrm{C} 85^{\prime}-\mathrm{C} 84^{\prime}$ & $102.7(14)$ \\
\hline $\mathrm{O} 1^{\prime}-\mathrm{C} 85^{\prime}-\mathrm{H} 85 \mathrm{C}$ & 111.2 \\
\hline $\mathrm{C} 84^{\prime}-\mathrm{C} 85^{\prime}-\mathrm{H} 85 \mathrm{C}$ & 111.2 \\
\hline $\mathrm{O} 1^{\prime}-\mathrm{C} 85^{\prime}-\mathrm{H} 85 \mathrm{D}$ & 111.2 \\
\hline $\mathrm{C} 84^{\prime}-\mathrm{C} 85^{\prime}-\mathrm{H} 85 \mathrm{D}$ & 111.2 \\
\hline $\mathrm{H} 85 \mathrm{C}-\mathrm{C} 85^{\prime}-\mathrm{H} 85 \mathrm{D}$ & 109.1 \\
\hline $\mathrm{C} 86-\mathrm{O} 2-\mathrm{C} 89$ & 102.5 \\
\hline
\end{tabular}




\begin{tabular}{|c|c|}
\hline $\mathrm{C} 43-\mathrm{C} 42-\mathrm{H} 42$ & 119.5 \\
\hline $\mathrm{C} 42-\mathrm{C} 43-\mathrm{C} 38$ & $118.6(4)$ \\
\hline $\mathrm{C} 42-\mathrm{C} 43-\mathrm{C} 45$ & $121.8(4)$ \\
\hline $\mathrm{C} 38-\mathrm{C} 43-\mathrm{C} 45$ & $119.6(3)$ \\
\hline $\mathrm{C} 39-\mathrm{C} 44-\mathrm{H} 44 \mathrm{~A}$ & 109.5 \\
\hline C39-C44-H44B & 109.5 \\
\hline $\mathrm{H} 44 \mathrm{~A}-\mathrm{C} 44-\mathrm{H} 44 \mathrm{~B}$ & 109.5 \\
\hline $\mathrm{C} 39-\mathrm{C} 44-\mathrm{H} 44 \mathrm{C}$ & 109.5 \\
\hline $\mathrm{H} 44 \mathrm{~A}-\mathrm{C} 44-\mathrm{H} 44 \mathrm{C}$ & 109.5 \\
\hline $\mathrm{H} 44 \mathrm{~B}-\mathrm{C} 44-\mathrm{H} 44 \mathrm{C}$ & 109.5 \\
\hline $\mathrm{C} 43-\mathrm{C} 45-\mathrm{H} 45 \mathrm{~A}$ & 109.5 \\
\hline $\mathrm{C} 43-\mathrm{C} 45-\mathrm{H} 45 \mathrm{~B}$ & 109.5 \\
\hline $\mathrm{H} 45 \mathrm{~A}-\mathrm{C} 45-\mathrm{H} 45 \mathrm{~B}$ & 109.5 \\
\hline $\mathrm{C} 43-\mathrm{C} 45-\mathrm{H} 45 \mathrm{C}$ & 109.5 \\
\hline $\mathrm{H} 45 \mathrm{~A}-\mathrm{C} 45-\mathrm{H} 45 \mathrm{C}$ & 109.5 \\
\hline $\mathrm{H} 45 \mathrm{~B}-\mathrm{C} 45-\mathrm{H} 45 \mathrm{C}$ & 109.5 \\
\hline $\mathrm{N} 6-\mathrm{C} 46-\mathrm{C} 55$ & $122.9(2)$ \\
\hline $\mathrm{N} 6-\mathrm{C} 46-\mathrm{Fe} 1$ & $118.09(17)$ \\
\hline $\mathrm{C} 55-\mathrm{C} 46-\mathrm{Fe} 1$ & $118.73(17)$ \\
\hline $\mathrm{C} 46-\mathrm{N} 6-\mathrm{C} 37$ & $116.62(19)$ \\
\hline $\mathrm{C} 46-\mathrm{N} 6-\mathrm{C} 47$ & $125.8(2)$ \\
\hline $\mathrm{C} 37-\mathrm{N} 6-\mathrm{C} 47$ & $116.94(19)$ \\
\hline $\mathrm{C} 48-\mathrm{C} 47-\mathrm{C} 52$ & $122.2(3)$ \\
\hline $\mathrm{C} 48-\mathrm{C} 47-\mathrm{N} 6$ & $118.0(2)$ \\
\hline $\mathrm{C} 52-\mathrm{C} 47-\mathrm{N} 6$ & $119.8(2)$ \\
\hline $\mathrm{C} 49-\mathrm{C} 48-\mathrm{C} 47$ & $118.0(3)$ \\
\hline $\mathrm{C} 49-\mathrm{C} 48-\mathrm{C} 53$ & $119.9(3)$ \\
\hline $\mathrm{C} 47-\mathrm{C} 48-\mathrm{C} 53$ & $122.1(3)$ \\
\hline $\mathrm{C} 50-\mathrm{C} 49-\mathrm{C} 48$ & $120.7(4)$ \\
\hline $\mathrm{C} 50-\mathrm{C} 49-\mathrm{H} 49$ & 119.7 \\
\hline $\mathrm{C} 48-\mathrm{C} 49-\mathrm{H} 49$ & 119.7 \\
\hline $\mathrm{C} 51-\mathrm{C} 50-\mathrm{C} 49$ & $121.0(4)$ \\
\hline $\mathrm{C} 51-\mathrm{C} 50-\mathrm{H} 50$ & 119.5 \\
\hline $\mathrm{C} 49-\mathrm{C} 50-\mathrm{H} 50$ & 119.5 \\
\hline $\mathrm{C} 50-\mathrm{C} 51-\mathrm{C} 52$ & $120.9(4)$ \\
\hline $\mathrm{C} 50-\mathrm{C} 51-\mathrm{H} 51$ & 119.6 \\
\hline $\mathrm{C} 52-\mathrm{C} 51-\mathrm{H} 51$ & 119.6 \\
\hline $\mathrm{C} 51-\mathrm{C} 52-\mathrm{C} 47$ & $117.2(3)$ \\
\hline $\mathrm{C} 51-\mathrm{C} 52-\mathrm{C} 54$ & $121.5(3)$ \\
\hline $\mathrm{C} 47-\mathrm{C} 52-\mathrm{C} 54$ & $121.3(3)$ \\
\hline $\mathrm{C} 48-\mathrm{C} 53-\mathrm{H} 53 \mathrm{~A}$ & 109.5 \\
\hline $\mathrm{C} 48-\mathrm{C} 53-\mathrm{H} 53 \mathrm{~B}$ & 109.5 \\
\hline $\mathrm{H} 53 \mathrm{~A}-\mathrm{C} 53-\mathrm{H} 53 \mathrm{~B}$ & 109.5 \\
\hline $\mathrm{C} 48-\mathrm{C} 53-\mathrm{H} 53 \mathrm{C}$ & 109.5 \\
\hline $\mathrm{H} 53 \mathrm{~A}-\mathrm{C} 53-\mathrm{H} 53 \mathrm{C}$ & 109.5 \\
\hline $\mathrm{H} 53 \mathrm{~B}-\mathrm{C} 53-\mathrm{H} 53 \mathrm{C}$ & 109.5 \\
\hline $\mathrm{C} 52-\mathrm{C} 54-\mathrm{H} 54 \mathrm{~A}$ & 109.5 \\
\hline 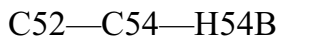 & \\
\hline
\end{tabular}

\begin{tabular}{|c|c|}
\hline $\mathrm{O} 2-\mathrm{C} 86-\mathrm{C} 87$ & $101.8(13)$ \\
\hline $\mathrm{O} 2-\mathrm{C} 86-\mathrm{H} 86 \mathrm{~A}$ & 111.4 \\
\hline $\mathrm{C} 87-\mathrm{C} 86-\mathrm{H} 86 \mathrm{~A}$ & 111.4 \\
\hline $\mathrm{O} 2-\mathrm{C} 86-\mathrm{H} 86 \mathrm{~B}$ & 111.4 \\
\hline $\mathrm{C} 87-\mathrm{C} 86-\mathrm{H} 86 \mathrm{~B}$ & 111.4 \\
\hline $\mathrm{H} 86 \mathrm{~A}-\mathrm{C} 86-\mathrm{H} 86 \mathrm{~B}$ & 109.3 \\
\hline $\mathrm{C} 86-\mathrm{C} 87-\mathrm{C} 88$ & $92.3(13)$ \\
\hline $\mathrm{C} 86-\mathrm{C} 87-\mathrm{H} 87 \mathrm{~A}$ & 113.2 \\
\hline $\mathrm{C} 88-\mathrm{C} 87-\mathrm{H} 87 \mathrm{~A}$ & 113.2 \\
\hline $\mathrm{C} 86-\mathrm{C} 87-\mathrm{H} 87 \mathrm{~B}$ & 113.2 \\
\hline C88-C87-H87B & 113.2 \\
\hline $\mathrm{H} 87 \mathrm{~A}-\mathrm{C} 87-\mathrm{H} 87 \mathrm{~B}$ & 110.6 \\
\hline $\mathrm{C} 87-\mathrm{C} 88-\mathrm{C} 89$ & $95.5(13)$ \\
\hline $\mathrm{C} 87-\mathrm{C} 88-\mathrm{H} 88 \mathrm{~A}$ & 112.6 \\
\hline $\mathrm{C} 89-\mathrm{C} 88-\mathrm{H} 88 \mathrm{~A}$ & 112.6 \\
\hline $\mathrm{C} 87-\mathrm{C} 88-\mathrm{H} 88 \mathrm{~B}$ & 112.6 \\
\hline $\mathrm{C} 89-\mathrm{C} 88-\mathrm{H} 88 \mathrm{~B}$ & 112.6 \\
\hline $\mathrm{H} 88 \mathrm{~A}-\mathrm{C} 88-\mathrm{H} 88 \mathrm{~B}$ & 110.1 \\
\hline $\mathrm{O} 2-\mathrm{C} 89-\mathrm{C} 88$ & $104.5(11)$ \\
\hline $\mathrm{O} 2-\mathrm{C} 89-\mathrm{H} 89 \mathrm{~A}$ & 110.9 \\
\hline $\mathrm{C} 88-\mathrm{C} 89-\mathrm{H} 89 \mathrm{~A}$ & 110.9 \\
\hline $\mathrm{O} 2-\mathrm{C} 89-\mathrm{H} 89 \mathrm{~B}$ & 110.9 \\
\hline С $88-\mathrm{C} 89-\mathrm{H} 89 \mathrm{~B}$ & 110.9 \\
\hline $\mathrm{H} 89 \mathrm{~A}-\mathrm{C} 89-\mathrm{H} 89 \mathrm{~B}$ & 108.9 \\
\hline $\mathrm{C} 89^{\prime}-\mathrm{O} 2^{\prime}-\mathrm{C} 86^{\prime}$ & $99.2(16)$ \\
\hline $\mathrm{O} 2^{\prime}-\mathrm{C} 86^{\prime}-\mathrm{C} 87^{\prime}$ & $104.8(16)$ \\
\hline $\mathrm{O} 2^{\prime}-\mathrm{C} 86^{\prime}-\mathrm{H} 86 \mathrm{C}$ & 110.8 \\
\hline $\mathrm{C} 87^{\prime}-\mathrm{C} 86^{\prime}-\mathrm{H} 86 \mathrm{C}$ & 110.8 \\
\hline $\mathrm{O} 2^{\prime}-\mathrm{C} 86^{\prime}-\mathrm{H} 86 \mathrm{D}$ & 110.8 \\
\hline $\mathrm{C} 87^{\prime}-\mathrm{C} 86^{\prime}-\mathrm{H} 86 \mathrm{D}$ & 110.8 \\
\hline $\mathrm{H} 86 \mathrm{C}-\mathrm{C} 86^{\prime}-\mathrm{H} 86 \mathrm{D}$ & 108.9 \\
\hline $\mathrm{C} 88^{\prime}-\mathrm{C} 87^{\prime}-\mathrm{C} 86^{\prime}$ & $89.5(15)$ \\
\hline $\mathrm{C} 88^{\prime}-\mathrm{C} 87^{\prime}-\mathrm{H} 87 \mathrm{C}$ & 113.7 \\
\hline $\mathrm{C} 86^{\prime}-\mathrm{C} 87^{\prime}-\mathrm{H} 87 \mathrm{C}$ & 113.7 \\
\hline $\mathrm{C} 88^{\prime}-\mathrm{C} 87^{\prime}-\mathrm{H} 87 \mathrm{D}$ & 113.7 \\
\hline $\mathrm{C} 86^{\prime}-\mathrm{C} 87^{\prime}-\mathrm{H} 87 \mathrm{D}$ & 113.7 \\
\hline $\mathrm{H} 87 \mathrm{C}-\mathrm{C} 87^{\prime}-\mathrm{H} 87 \mathrm{D}$ & 111.0 \\
\hline $\mathrm{C} 87^{\prime}-\mathrm{C} 88^{\prime}-\mathrm{C} 89^{\prime}$ & $89.8(15)$ \\
\hline $\mathrm{C} 87^{\prime}-\mathrm{C} 88^{\prime}-\mathrm{H} 88 \mathrm{C}$ & 113.7 \\
\hline $\mathrm{C} 89^{\prime}-\mathrm{C} 88^{\prime}-\mathrm{H} 88 \mathrm{C}$ & 113.7 \\
\hline $\mathrm{C} 87^{\prime}-\mathrm{C} 88^{\prime}-\mathrm{H} 88 \mathrm{D}$ & 113.7 \\
\hline $\mathrm{C} 89^{\prime}-\mathrm{C} 88^{\prime}-\mathrm{H} 88 \mathrm{D}$ & 113.7 \\
\hline $\mathrm{H} 88 \mathrm{C}-\mathrm{C} 88^{\prime}-\mathrm{H} 88 \mathrm{D}$ & 110.9 \\
\hline $\mathrm{O} 2^{\prime}-\mathrm{C} 89^{\prime}-\mathrm{C} 88^{\prime}$ & $99.6(15)$ \\
\hline $\mathrm{O} 2^{\prime}-\mathrm{C} 89^{\prime}-\mathrm{H} 89 \mathrm{C}$ & 111.8 \\
\hline $\mathrm{C} 88^{\prime}-\mathrm{C} 89^{\prime}-\mathrm{H} 89 \mathrm{C}$ & 111.8 \\
\hline $\mathrm{O} 2^{\prime}-\mathrm{C} 89^{\prime}-\mathrm{H} 89 \mathrm{D}$ & 111.8 \\
\hline $\mathrm{C} 88^{\prime}-\mathrm{C} 89^{\prime}-\mathrm{H} 89 \mathrm{D}$ & 111.8 \\
\hline
\end{tabular}




\begin{tabular}{|c|c|c|c|}
\hline $\mathrm{H} 54 \mathrm{~A}-\mathrm{C} 54-\mathrm{H} 54 \mathrm{~B}$ & 109.5 & $\mathrm{H} 89 \mathrm{C}-\mathrm{C} 89^{\prime}-\mathrm{H} 89 \mathrm{D}$ & 109.6 \\
\hline $\mathrm{C} 10-\mathrm{Fe} 1-\mathrm{C} 1-\mathrm{N} 1$ & $-34(7)$ & $\mathrm{C} 53-\mathrm{C} 48-\mathrm{C} 49-\mathrm{C} 50$ & $178.5(4)$ \\
\hline $\mathrm{C} 46-\mathrm{Fe} 1-\mathrm{C} 1-\mathrm{N} 1$ & $146(7)$ & $\mathrm{C} 48-\mathrm{C} 49-\mathrm{C} 50-\mathrm{C} 51$ & $0.0(6)$ \\
\hline $\mathrm{C} 73-\mathrm{Fe} 1-\mathrm{C} 1-\mathrm{N} 1$ & $64(7)$ & $\mathrm{C} 49-\mathrm{C} 50-\mathrm{C} 51-\mathrm{C} 52$ & $0.8(6)$ \\
\hline $\mathrm{C} 28-\mathrm{Fe} 1-\mathrm{C} 1-\mathrm{N} 1$ & $-133(7)$ & $\mathrm{C} 50-\mathrm{C} 51-\mathrm{C} 52-\mathrm{C} 47$ & $-0.6(5)$ \\
\hline $\mathrm{C} 7-\mathrm{C} 2-\mathrm{C} 3-\mathrm{C} 4$ & $-1(3)$ & $\mathrm{C} 50-\mathrm{C} 51-\mathrm{C} 52-\mathrm{C} 54$ & $177.8(3)$ \\
\hline $\mathrm{N} 1-\mathrm{C} 2-\mathrm{C} 3-\mathrm{C} 4$ & $175.9(18)$ & $\mathrm{C} 48-\mathrm{C} 47-\mathrm{C} 52-\mathrm{C} 51$ & $-0.5(4)$ \\
\hline $\mathrm{C} 7-\mathrm{C} 2-\mathrm{C} 3-\mathrm{C} 8$ & $178(2)$ & N6-C47-C52-C51 & $179.0(2)$ \\
\hline $\mathrm{N} 1-\mathrm{C} 2-\mathrm{C} 3-\mathrm{C} 8$ & $-5(3)$ & $\mathrm{C} 48-\mathrm{C} 47-\mathrm{C} 52-\mathrm{C} 54$ & $-178.9(3)$ \\
\hline $\mathrm{C} 2-\mathrm{C} 3-\mathrm{C} 4-\mathrm{C} 5$ & $0(2)$ & $\mathrm{N} 6-\mathrm{C} 47-\mathrm{C} 52-\mathrm{C} 54$ & $0.6(4)$ \\
\hline $\mathrm{C} 8-\mathrm{C} 3-\mathrm{C} 4-\mathrm{C} 5$ & $-179(2)$ & N6-C46-C55-C64 & $-168.7(2)$ \\
\hline $\mathrm{C} 3-\mathrm{C} 4-\mathrm{C} 5-\mathrm{C} 6$ & $2(2)$ & $\mathrm{Fe} 1-\mathrm{C} 46-\mathrm{C} 55-\mathrm{C} 64$ & $5.4(3)$ \\
\hline $\mathrm{C} 4-\mathrm{C} 5-\mathrm{C} 6-\mathrm{C} 7$ & $-2(2)$ & $\mathrm{N} 6-\mathrm{C} 46-\mathrm{C} 55-\mathrm{N} 7$ & $12.7(4)$ \\
\hline $\mathrm{C} 5-\mathrm{C} 6-\mathrm{C} 7-\mathrm{C} 2$ & $1(2)$ & $\mathrm{Fe} 1-\mathrm{C} 46-\mathrm{C} 55-\mathrm{N} 7$ & $-173.24(18)$ \\
\hline $\mathrm{C} 5-\mathrm{C} 6-\mathrm{C} 7-\mathrm{C} 9$ & $175.2(18)$ & $\mathrm{C} 64-\mathrm{C} 55-\mathrm{N} 7-\mathrm{C} 56$ & $-46.0(4)$ \\
\hline $\mathrm{C} 3-\mathrm{C} 2-\mathrm{C} 7-\mathrm{C} 6$ & $1(3)$ & $\mathrm{C} 46-\mathrm{C} 55-\mathrm{N} 7-\mathrm{C} 56$ & $132.5(3)$ \\
\hline $\mathrm{N} 1-\mathrm{C} 2-\mathrm{C} 7-\mathrm{C} 6$ & $-176.5(18)$ & $\mathrm{C} 55-\mathrm{N} 7-\mathrm{C} 56-\mathrm{C} 61$ & $-28.1(4)$ \\
\hline $\mathrm{C} 3-\mathrm{C} 2-\mathrm{C} 7-\mathrm{C} 9$ & $-172(2)$ & $\mathrm{C} 55-\mathrm{N} 7-\mathrm{C} 56-\mathrm{C} 57$ & $154.8(2)$ \\
\hline $\mathrm{N} 1-\mathrm{C} 2-\mathrm{C} 7-\mathrm{C} 9$ & $10(3)$ & $\mathrm{N} 7-\mathrm{C} 56-\mathrm{C} 57-\mathrm{C} 58$ & $-177.0(3)$ \\
\hline $\mathrm{C} 7^{\prime}-\mathrm{C} 2^{\prime}-\mathrm{C} 3^{\prime}-\mathrm{C} 4^{\prime}$ & $2(2)$ & $\mathrm{C} 61-\mathrm{C} 56-\mathrm{C} 57-\mathrm{C} 58$ & $5.8(4)$ \\
\hline $\mathrm{N} 1^{\prime}-\mathrm{C} 2^{\prime}-\mathrm{C} 3^{\prime}-\mathrm{C} 4^{\prime}$ & $-175.7(17)$ & $\mathrm{N} 7-\mathrm{C} 56-\mathrm{C} 57-\mathrm{C} 62$ & $4.0(4)$ \\
\hline $\mathrm{C} 7^{\prime}-\mathrm{C} 2^{\prime}-\mathrm{C} 3^{\prime}-\mathrm{C} 8^{\prime}$ & $-174(2)$ & $\mathrm{C} 61-\mathrm{C} 56-\mathrm{C} 57-\mathrm{C} 62$ & $-173.2(3)$ \\
\hline $\mathrm{N} 1^{\prime}-\mathrm{C} 2^{\prime}-\mathrm{C} 3^{\prime}-\mathrm{C} 8^{\prime}$ & $8(3)$ & $\mathrm{C} 56-\mathrm{C} 57-\mathrm{C} 58-\mathrm{C} 59$ & $-0.6(5)$ \\
\hline $\mathrm{C} 2^{\prime}-\mathrm{C} 3^{\prime}-\mathrm{C} 4^{\prime}-\mathrm{C} 5^{\prime}$ & $0(2)$ & $\mathrm{C} 62-\mathrm{C} 57-\mathrm{C} 58-\mathrm{C} 59$ & $178.5(3)$ \\
\hline $\mathrm{C} 8^{\prime}-\mathrm{C} 3^{\prime}-\mathrm{C} 4^{\prime}-\mathrm{C} 5^{\prime}$ & $176(2)$ & $\mathrm{C} 57-\mathrm{C} 58-\mathrm{C} 59-\mathrm{C} 60$ & $-3.7(6)$ \\
\hline $\mathrm{C} 3^{\prime}-\mathrm{C} 4^{\prime}-\mathrm{C} 5^{\prime}-\mathrm{C} 6^{\prime}$ & $-2.3(19)$ & $\mathrm{C} 58-\mathrm{C} 59-\mathrm{C} 60-\mathrm{C} 61$ & $2.8(6)$ \\
\hline $\mathrm{C} 4^{\prime}-\mathrm{C} 5^{\prime}-\mathrm{C} 6^{\prime}-\mathrm{C} 7^{\prime}$ & $2.9(19)$ & $\mathrm{C} 59-\mathrm{C} 60-\mathrm{C} 61-\mathrm{C} 56$ & $2.4(5)$ \\
\hline $\mathrm{C} 5^{\prime}-\mathrm{C} 6^{\prime}-\mathrm{C} 7^{\prime}-\mathrm{C} 2^{\prime}$ & $-1(2)$ & $\mathrm{C} 59-\mathrm{C} 60-\mathrm{C} 61-\mathrm{C} 63$ & $-174.8(3)$ \\
\hline $\mathrm{C} 5^{\prime}-\mathrm{C} 6^{\prime}-\mathrm{C} 7^{\prime}-\mathrm{C} 9^{\prime}$ & $-174.8(17)$ & $\mathrm{N} 7-\mathrm{C} 56-\mathrm{C} 61-\mathrm{C} 60$ & $176.3(3)$ \\
\hline $\mathrm{C} 3^{\prime}-\mathrm{C} 2^{\prime}-\mathrm{C} 7^{\prime}-\mathrm{C}^{\prime}$ & $-2(2)$ & $\mathrm{C} 57-\mathrm{C} 56-\mathrm{C} 61-\mathrm{C} 60$ & $-6.7(4)$ \\
\hline $\mathrm{N} 1^{\prime}-\mathrm{C} 2^{\prime}-\mathrm{C} 7^{\prime}-\mathrm{C} 6^{\prime}$ & $176.2(17)$ & $\mathrm{N} 7-\mathrm{C} 56-\mathrm{C} 61-\mathrm{C} 63$ & $-6.7(4)$ \\
\hline $\mathrm{C} 3^{\prime}-\mathrm{C} 2^{\prime}-\mathrm{C} 7^{\prime}-\mathrm{C} 9^{\prime}$ & $172.5(19)$ & $\mathrm{C} 57-\mathrm{C} 56-\mathrm{C} 61-\mathrm{C} 63$ & $170.3(3)$ \\
\hline $\mathrm{N} 1^{\prime}-\mathrm{C} 2^{\prime}-\mathrm{C} 7^{\prime}-\mathrm{C} 9^{\prime}$ & $-9(3)$ & $\mathrm{C} 46-\mathrm{C} 55-\mathrm{C} 64-\mathrm{N} 8^{\prime}$ & $170.7(13)$ \\
\hline $\mathrm{C} 16-\mathrm{C} 11-\mathrm{C} 12-\mathrm{C} 13$ & $-0.3(5)$ & $\mathrm{N} 7-\mathrm{C} 55-\mathrm{C} 64-\mathrm{N} 8{ }^{\prime}$ & $-10.6(13)$ \\
\hline $\mathrm{N} 2-\mathrm{C} 11-\mathrm{C} 12-\mathrm{C} 13$ & $178.9(3)$ & $\mathrm{C} 46-\mathrm{C} 55-\mathrm{C} 64-\mathrm{N} 8$ & $164.7(12)$ \\
\hline $\mathrm{C} 16-\mathrm{C} 11-\mathrm{C} 12-\mathrm{C} 17$ & $179.4(3)$ & $\mathrm{N} 7-\mathrm{C} 55-\mathrm{C} 64-\mathrm{N} 8$ & $-16.6(12)$ \\
\hline $\mathrm{N} 2-\mathrm{C} 11-\mathrm{C} 12-\mathrm{C} 17$ & $-1.4(4)$ & $\mathrm{C} 46-\mathrm{C} 55-\mathrm{C} 64-\mathrm{C} 73$ & $-17.8(3)$ \\
\hline $\mathrm{C} 11-\mathrm{C} 12-\mathrm{C} 13-\mathrm{C} 14$ & $-0.5(6)$ & $\mathrm{N} 7-\mathrm{C} 55-\mathrm{C} 64-\mathrm{C} 73$ & $160.9(2)$ \\
\hline $\mathrm{C} 17-\mathrm{C} 12-\mathrm{C} 13-\mathrm{C} 14$ & $179.8(4)$ & $\mathrm{C} 55-\mathrm{C} 64-\mathrm{N} 8-\mathrm{C} 65$ & $0(3)$ \\
\hline $\mathrm{C} 12-\mathrm{C} 13-\mathrm{C} 14-\mathrm{C} 15$ & $0.6(7)$ & $\mathrm{C} 73-\mathrm{C} 64-\mathrm{N} 8-\mathrm{C} 65$ & $-177.6(17)$ \\
\hline $\mathrm{C} 13-\mathrm{C} 14-\mathrm{C} 15-\mathrm{C} 16$ & $0.0(6)$ & $\mathrm{C} 64-\mathrm{N} 8-\mathrm{C} 65-\mathrm{C} 66$ & $-64(4)$ \\
\hline $\mathrm{N} 2-\mathrm{C} 11-\mathrm{C} 16-\mathrm{C} 15$ & $-178.2(3)$ & $\mathrm{C} 64-\mathrm{N} 8-\mathrm{C} 65-\mathrm{C} 70$ & $126(3)$ \\
\hline $\mathrm{C} 12-\mathrm{C} 11-\mathrm{C} 16-\mathrm{C} 15$ & $0.9(5)$ & $\mathrm{C} 70-\mathrm{C} 65-\mathrm{C} 66-\mathrm{C} 67$ & $-4(4)$ \\
\hline $\mathrm{N} 2-\mathrm{C} 11-\mathrm{C} 16-\mathrm{C} 18$ & $1.2(4)$ & $\mathrm{N} 8-\mathrm{C} 65-\mathrm{C} 66-\mathrm{C} 67$ & $-173(3)$ \\
\hline $\mathrm{C} 12-\mathrm{C} 11-\mathrm{C} 16-\mathrm{C} 18$ & $-179.6(3)$ & $\mathrm{C} 70-\mathrm{C} 65-\mathrm{C} 66-\mathrm{C} 71$ & $170(3)$ \\
\hline $\mathrm{C} 14-\mathrm{C} 15-\mathrm{C} 16-\mathrm{C} 11$ & $-0.7(5)$ & $\mathrm{N} 8-\mathrm{C} 65-\mathrm{C} 66-\mathrm{C} 71$ & $1(4)$ \\
\hline $\mathrm{C} 14-\mathrm{C} 15-\mathrm{C} 16-\mathrm{C} 18$ & $179.8(3)$ & $\mathrm{C} 65-\mathrm{C} 66-\mathrm{C} 67-\mathrm{C} 68$ & $-1(3)$ \\
\hline
\end{tabular}




\begin{tabular}{|c|c|c|c|}
\hline $\mathrm{C} 25-\mathrm{C} 20-\mathrm{C} 21-\mathrm{C} 22$ & $0.0(6)$ & $\mathrm{C} 71-\mathrm{C} 66-\mathrm{C} 67-\mathrm{C} 68$ & $-175.4(17)$ \\
\hline $\mathrm{N} 3-\mathrm{C} 20-\mathrm{C} 21-\mathrm{C} 22$ & $177.8(4)$ & $\mathrm{C} 66-\mathrm{C} 67-\mathrm{C} 68-\mathrm{C} 69$ & $3(2)$ \\
\hline $\mathrm{C} 25-\mathrm{C} 20-\mathrm{C} 21-\mathrm{C} 26$ & $-177.7(4)$ & $\mathrm{C} 67-\mathrm{C} 68-\mathrm{C} 69-\mathrm{C} 70$ & $1(2)$ \\
\hline $\mathrm{N} 3-\mathrm{C} 20-\mathrm{C} 21-\mathrm{C} 26$ & $0.1(6)$ & $\mathrm{C} 68-\mathrm{C} 69-\mathrm{C} 70-\mathrm{C} 65$ & $-6(3)$ \\
\hline $\mathrm{C} 20-\mathrm{C} 21-\mathrm{C} 22-\mathrm{C} 23$ & $0.4(8)$ & $\mathrm{C} 68-\mathrm{C} 69-\mathrm{C} 70-\mathrm{C} 72$ & $178.2(19)$ \\
\hline $\mathrm{C} 26-\mathrm{C} 21-\mathrm{C} 22-\mathrm{C} 23$ & $178.1(6)$ & $\mathrm{C} 66-\mathrm{C} 65-\mathrm{C} 70-\mathrm{C} 69$ & $7(4)$ \\
\hline $\mathrm{C} 21-\mathrm{C} 22-\mathrm{C} 23-\mathrm{C} 24$ & $-0.1(11)$ & $\mathrm{N} 8-\mathrm{C} 65-\mathrm{C} 70-\mathrm{C} 69$ & $177(2)$ \\
\hline $\mathrm{C} 22-\mathrm{C} 23-\mathrm{C} 24-\mathrm{C} 25$ & $-0.7(10)$ & $\mathrm{C} 66-\mathrm{C} 65-\mathrm{C} 70-\mathrm{C} 72$ & $-177(3)$ \\
\hline $\mathrm{C} 21-\mathrm{C} 20-\mathrm{C} 25-\mathrm{C} 24$ & $-0.8(6)$ & $\mathrm{N} 8-\mathrm{C} 65-\mathrm{C} 70-\mathrm{C} 72$ & $-7(4)$ \\
\hline $\mathrm{N} 3-\mathrm{C} 20-\mathrm{C} 25-\mathrm{C} 24$ & $-178.6(3)$ & $\mathrm{C} 55-\mathrm{C} 64-\mathrm{N} 8^{\prime}-\mathrm{C} 65^{\prime}$ & $-26(4)$ \\
\hline $\mathrm{C} 21-\mathrm{C} 20-\mathrm{C} 25-\mathrm{C} 27$ & $178.6(4)$ & $\mathrm{C} 73-\mathrm{C} 64-\mathrm{N} 8^{\prime}-\mathrm{C}^{\prime} 5^{\prime}$ & $162(3)$ \\
\hline $\mathrm{N} 3-\mathrm{C} 20-\mathrm{C} 25-\mathrm{C} 27$ & $0.8(6)$ & $\mathrm{C} 64-\mathrm{N} 8^{\prime}-\mathrm{C} 65^{\prime}-\mathrm{C} 66^{\prime}$ & $-40(7)$ \\
\hline $\mathrm{C} 23-\mathrm{C} 24-\mathrm{C} 25-\mathrm{C} 20$ & $1.1(8)$ & $\mathrm{C} 64-\mathrm{N} 8^{\prime}-\mathrm{C} 65^{\prime}-\mathrm{C} 70^{\prime}$ & $139(3)$ \\
\hline $\mathrm{C} 23-\mathrm{C} 24-\mathrm{C} 25-\mathrm{C} 27$ & $-178.2(6)$ & $\mathrm{C} 70^{\prime}-\mathrm{C} 65^{\prime}-\mathrm{C} 66^{\prime}-\mathrm{C} 67^{\prime}$ & $-4(6)$ \\
\hline $\mathrm{C} 37-\mathrm{C} 28-\mathrm{N} 4-\mathrm{C} 29$ & $179.6(2)$ & $\mathrm{N} 8^{\prime}-\mathrm{C} 65^{\prime}-\mathrm{C} 66^{\prime}-\mathrm{C} 67^{\prime}$ & $174(4)$ \\
\hline $\mathrm{Fe} 1-\mathrm{C} 28-\mathrm{N} 4-\mathrm{C} 29$ & $-7.5(4)$ & $\mathrm{C} 70^{\prime}-\mathrm{C} 65^{\prime}-\mathrm{C} 66^{\prime}-\mathrm{C} 71^{\prime}$ & $179(4)$ \\
\hline $\mathrm{C} 28-\mathrm{N} 4-\mathrm{C} 29-\mathrm{C} 34$ & $-81.9(3)$ & $\mathrm{N} 8^{\prime}-\mathrm{C} 65^{\prime}-\mathrm{C} 66^{\prime}-\mathrm{C} 71^{\prime}$ & $-2(6)$ \\
\hline $\mathrm{C} 28-\mathrm{N} 4-\mathrm{C} 29-\mathrm{C} 30$ & $107.2(3)$ & $\mathrm{C} 65^{\prime}-\mathrm{C} 66^{\prime}-\mathrm{C} 67^{\prime}-\mathrm{C} 68^{\prime}$ & $6(4)$ \\
\hline $\mathrm{C} 34-\mathrm{C} 29-\mathrm{C} 30-\mathrm{C} 31$ & $4.1(4)$ & $\mathrm{C} 71^{\prime}-\mathrm{C} 66^{\prime}-\mathrm{C} 67^{\prime}-\mathrm{C} 68^{\prime}$ & $-177(2)$ \\
\hline $\mathrm{N} 4-\mathrm{C} 29-\mathrm{C} 30-\mathrm{C} 31$ & $174.9(3)$ & $\mathrm{C} 66^{\prime}-\mathrm{C} 67^{\prime}-\mathrm{C} 68^{\prime}-\mathrm{C} 69^{\prime}$ & $-4(4)$ \\
\hline $\mathrm{C} 34-\mathrm{C} 29-\mathrm{C} 30-\mathrm{C} 35$ & $-171.7(3)$ & $\mathrm{C} 67^{\prime}-\mathrm{C} 68^{\prime}-\mathrm{C} 69^{\prime}-\mathrm{C} 70^{\prime}$ & $-2(3)$ \\
\hline $\mathrm{N} 4-\mathrm{C} 29-\mathrm{C} 30-\mathrm{C} 35$ & $-0.8(4)$ & $\mathrm{C} 68^{\prime}-\mathrm{C} 69^{\prime}-\mathrm{C} 70^{\prime}-\mathrm{C} 65^{\prime}$ & $4(4)$ \\
\hline $\mathrm{C} 29-\mathrm{C} 30-\mathrm{C} 31-\mathrm{C} 32$ & $-1.3(5)$ & $\mathrm{C} 68^{\prime}-\mathrm{C} 69^{\prime}-\mathrm{C} 70^{\prime}-\mathrm{C} 72^{\prime}$ & $177(3)$ \\
\hline $\mathrm{C} 35-\mathrm{C} 30-\mathrm{C} 31-\mathrm{C} 32$ & $174.4(3)$ & $\mathrm{C} 66^{\prime}-\mathrm{C} 65^{\prime}-\mathrm{C} 70^{\prime}-\mathrm{C} 69^{\prime}$ & $-1(6)$ \\
\hline $\mathrm{C} 30-\mathrm{C} 31-\mathrm{C} 32-\mathrm{C} 33$ & $-1.9(6)$ & $\mathrm{N} 8^{\prime}-\mathrm{C} 65^{\prime}-\mathrm{C} 70^{\prime}-\mathrm{C} 69^{\prime}$ & $-180(3)$ \\
\hline $\mathrm{C} 31-\mathrm{C} 32-\mathrm{C} 33-\mathrm{C} 34$ & $2.4(5)$ & $\mathrm{C} 66^{\prime}-\mathrm{C} 65^{\prime}-\mathrm{C} 70^{\prime}-\mathrm{C} 72^{\prime}$ & $-174(4)$ \\
\hline $\mathrm{C} 30-\mathrm{C} 29-\mathrm{C} 34-\mathrm{C} 33$ & $-3.5(4)$ & $\mathrm{N} 8^{\prime}-\mathrm{C} 65^{\prime}-\mathrm{C} 70^{\prime}-\mathrm{C} 72^{\prime}$ & $7(6)$ \\
\hline $\mathrm{N} 4-\mathrm{C} 29-\mathrm{C} 34-\mathrm{C} 33$ & $-174.3(2)$ & $\mathrm{N} 8{ }^{\prime}-\mathrm{C} 64-\mathrm{C} 73-\mathrm{N} 9$ & $16.8(13)$ \\
\hline $\mathrm{C} 30-\mathrm{C} 29-\mathrm{C} 34-\mathrm{C} 36$ & $174.3(3)$ & $\mathrm{N} 8-\mathrm{C} 64-\mathrm{C} 73-\mathrm{N} 9$ & $22.9(9)$ \\
\hline $\mathrm{N} 4-\mathrm{C} 29-\mathrm{C} 34-\mathrm{C} 36$ & $3.6(4)$ & $\mathrm{C} 55-\mathrm{C} 64-\mathrm{C} 73-\mathrm{N} 9$ & $-155.2(2)$ \\
\hline $\mathrm{C} 32-\mathrm{C} 33-\mathrm{C} 34-\mathrm{C} 29$ & $0.3(5)$ & $\mathrm{N} 8{ }^{\prime}-\mathrm{C} 64-\mathrm{C} 73-\mathrm{Fe} 1$ & $-166.2(13)$ \\
\hline $\mathrm{C} 32-\mathrm{C} 33-\mathrm{C} 34-\mathrm{C} 36$ & $-177.6(3)$ & $\mathrm{N} 8-\mathrm{C} 64-\mathrm{C} 73-\mathrm{Fe} 1$ & $-160.1(9)$ \\
\hline $\mathrm{N} 4-\mathrm{C} 28-\mathrm{C} 37-\mathrm{N} 5$ & $-11.3(4)$ & $\mathrm{C} 55-\mathrm{C} 64-\mathrm{C} 73-\mathrm{Fe} 1$ & $21.8(2)$ \\
\hline $\mathrm{Fe} 1-\mathrm{C} 28-\mathrm{C} 37-\mathrm{N} 5$ & $173.9(2)$ & $\mathrm{C} 64-\mathrm{C} 73-\mathrm{N} 9-\mathrm{C} 74$ & $175.9(2)$ \\
\hline $\mathrm{N} 4-\mathrm{C} 28-\mathrm{C} 37-\mathrm{N} 6$ & $170.2(2)$ & $\mathrm{Fe} 1-\mathrm{C} 73-\mathrm{N} 9-\mathrm{C} 74$ & $0.3(4)$ \\
\hline $\mathrm{Fe} 1-\mathrm{C} 28-\mathrm{C} 37-\mathrm{N} 6$ & $-4.6(2)$ & $\mathrm{C} 73-\mathrm{N} 9-\mathrm{C} 74-\mathrm{C} 75$ & $-104.7(3)$ \\
\hline $\mathrm{N} 6-\mathrm{C} 37-\mathrm{N} 5-\mathrm{C} 38$ & $-179.2(2)$ & $\mathrm{C} 73-\mathrm{N} 9-\mathrm{C} 74-\mathrm{C} 79$ & $86.6(3)$ \\
\hline $\mathrm{C} 28-\mathrm{C} 37-\mathrm{N} 5-\mathrm{C} 38$ & $2.5(5)$ & $\mathrm{C} 79-\mathrm{C} 74-\mathrm{C} 75-\mathrm{C} 76$ & $-2.3(4)$ \\
\hline $\mathrm{C} 37-\mathrm{N} 5-\mathrm{C} 38-\mathrm{C} 39$ & $-95.2(3)$ & $\mathrm{N} 9-\mathrm{C} 74-\mathrm{C} 75-\mathrm{C} 76$ & $-170.9(2)$ \\
\hline $\mathrm{C} 37-\mathrm{N} 5-\mathrm{C} 38-\mathrm{C} 43$ & $96.0(3)$ & $\mathrm{C} 79-\mathrm{C} 74-\mathrm{C} 75-\mathrm{C} 80$ & $174.5(3)$ \\
\hline $\mathrm{C} 43-\mathrm{C} 38-\mathrm{C} 39-\mathrm{C} 40$ & $-2.6(4)$ & $\mathrm{N} 9-\mathrm{C} 74-\mathrm{C} 75-\mathrm{C} 80$ & $5.8(4)$ \\
\hline $\mathrm{N} 5-\mathrm{C} 38-\mathrm{C} 39-\mathrm{C} 40$ & $-171.2(3)$ & $\mathrm{C} 74-\mathrm{C} 75-\mathrm{C} 76-\mathrm{C} 77$ & $0.6(5)$ \\
\hline $\mathrm{C} 43-\mathrm{C} 38-\mathrm{C} 39-\mathrm{C} 44$ & $176.0(3)$ & $\mathrm{C} 80-\mathrm{C} 75-\mathrm{C} 76-\mathrm{C} 77$ & $-176.2(3)$ \\
\hline $\mathrm{N} 5-\mathrm{C} 38-\mathrm{C} 39-\mathrm{C} 44$ & $7.4(4)$ & $\mathrm{C} 75-\mathrm{C} 76-\mathrm{C} 77-\mathrm{C} 78$ & $0.2(5)$ \\
\hline $\mathrm{C} 38-\mathrm{C} 39-\mathrm{C} 40-\mathrm{C} 41$ & $1.3(6)$ & $\mathrm{C} 76-\mathrm{C} 77-\mathrm{C} 78-\mathrm{C} 79$ & $0.7(5)$ \\
\hline $\mathrm{C} 44-\mathrm{C} 39-\mathrm{C} 40-\mathrm{C} 41$ & $-177.3(4)$ & $\mathrm{C} 77-\mathrm{C} 78-\mathrm{C} 79-\mathrm{C} 74$ & $-2.3(4)$ \\
\hline $\mathrm{C} 39-\mathrm{C} 40-\mathrm{C} 41-\mathrm{C} 42$ & $0.8(7)$ & $\mathrm{C} 77-\mathrm{C} 78-\mathrm{C} 79-\mathrm{C} 81$ & $173.0(3)$ \\
\hline $\mathrm{C} 40-\mathrm{C} 41-\mathrm{C} 42-\mathrm{C} 43$ & $-1.6(7)$ & $\mathrm{C} 75-\mathrm{C} 74-\mathrm{C} 79-\mathrm{C} 78$ & $3.1(4)$ \\
\hline
\end{tabular}




$\begin{array}{llll}\mathrm{C} 41-\mathrm{C} 42-\mathrm{C} 43-\mathrm{C} 38 & 0.2(6) & \mathrm{N} 9-\mathrm{C} 74-\mathrm{C} 79-\mathrm{C} 78 & 171.4(2) \\ \mathrm{C} 41-\mathrm{C} 42-\mathrm{C} 43-\mathrm{C} 45 & 179.4(4) & \mathrm{C} 75-\mathrm{C} 74-\mathrm{C} 79-\mathrm{C} 81 & -172.1(2) \\ \mathrm{C} 39-\mathrm{C} 38-\mathrm{C} 43-\mathrm{C} 42 & 1.9(5) & \mathrm{N} 9-\mathrm{C} 74-\mathrm{C} 79-\mathrm{C} 81 & -3.8(4) \\ \mathrm{N} 5-\mathrm{C} 38-\mathrm{C} 43-\mathrm{C} 42 & 170.6(3) & \mathrm{C} 85-\mathrm{O} 1-\mathrm{C} 82-\mathrm{C} 83 & 18(3) \\ \mathrm{C} 39-\mathrm{C} 38-\mathrm{C} 43-\mathrm{C} 45 & -177.3(3) & \mathrm{O} 1-\mathrm{C} 82-\mathrm{C} 83-\mathrm{C} 84 & 0(2) \\ \mathrm{N} 5-\mathrm{C} 38-\mathrm{C} 43-\mathrm{C} 45 & -8.6(4) & \mathrm{C} 82-\mathrm{C} 83-\mathrm{C} 84-\mathrm{C} 85 & -17(2) \\ \mathrm{C} 55-\mathrm{C} 46-\mathrm{N} 6-\mathrm{C} 37 & 178.3(2) & \mathrm{C} 82-\mathrm{O} 1-\mathrm{C} 85-\mathrm{C} 84 & -29(3) \\ \mathrm{Fe} 1-\mathrm{C} 46-\mathrm{N} 6-\mathrm{C} 37 & 4.1(3) & \mathrm{C} 83-\mathrm{C} 84-\mathrm{C} 85-\mathrm{O} 1 & 27(2) \\ \mathrm{C} 55-\mathrm{C} 46-\mathrm{N} 6-\mathrm{C} 47 & 7.4(4) & \mathrm{C} 85^{\prime}-\mathrm{O} 1^{\prime}-\mathrm{C} 82^{\prime}-\mathrm{C} 83^{\prime} & -36(3) \\ \mathrm{Fe} 1-\mathrm{C} 46-\mathrm{N} 6-\mathrm{C} 47 & -166.72(18) & \mathrm{O} 1^{\prime}-\mathrm{C} 82^{\prime}-\mathrm{C} 83^{\prime}-\mathrm{C} 84^{\prime} & 37(3) \\ \mathrm{N} 5-\mathrm{C} 37-\mathrm{N} 6-\mathrm{C} 46 & -178.2(2) & \mathrm{C} 82^{\prime}-\mathrm{C} 83^{\prime}-\mathrm{C} 84^{\prime}-\mathrm{C} 85^{\prime} & -27(3) \\ \mathrm{C} 28-\mathrm{C} 37-\mathrm{N} 6-\mathrm{C} 46 & 0.5(3) & \mathrm{C} 82^{\prime}-\mathrm{O} 1^{\prime}-\mathrm{C} 85^{\prime}-\mathrm{C} 84^{\prime} & 19(4) \\ \mathrm{N} 5-\mathrm{C} 37-\mathrm{N} 6-\mathrm{C} 47 & -6.5(3) & \mathrm{C} 83^{\prime}-\mathrm{C} 84^{\prime}-\mathrm{C} 85^{\prime}-\mathrm{O} 1^{\prime} & 5(4) \\ \mathrm{C} 28-\mathrm{C} 37-\mathrm{N} 6-\mathrm{C} 47 & 172.2(2) & \mathrm{C} 89-\mathrm{O} 2-\mathrm{C} 86-\mathrm{C} 87 & 33(2) \\ \mathrm{C} 46-\mathrm{N} 6-\mathrm{C} 47-\mathrm{C} 48 & 83.3(3) & \mathrm{O} 2-\mathrm{C} 86-\mathrm{C} 87-\mathrm{C} 88 & -60(2) \\ \mathrm{C} 37-\mathrm{N} 6-\mathrm{C} 47-\mathrm{C} 48 & -87.5(3) & \mathrm{C} 86-\mathrm{C} 87-\mathrm{C} 88-\mathrm{C} 89 & 60(2) \\ \mathrm{C} 46-\mathrm{N} 6-\mathrm{C} 47-\mathrm{C} 52 & -96.2(3) & \mathrm{C} 86-\mathrm{O} 2-\mathrm{C} 89-\mathrm{C} 88 & 6(2) \\ \mathrm{C} 37-\mathrm{N} 6-\mathrm{C} 47-\mathrm{C} 52 & 93.0(3) & \mathrm{C} 87-\mathrm{C} 88-\mathrm{C} 89-\mathrm{O} 2 & -43(2) \\ \mathrm{C} 52-\mathrm{C} 47-\mathrm{C} 48-\mathrm{C} 49 & 1.2(4) & \mathrm{C} 89^{\prime}-\mathrm{O} 2^{\prime}-\mathrm{C} 86^{\prime}-\mathrm{C} 87^{\prime} & -11(3) \\ \mathrm{N} 6-\mathrm{C} 47-\mathrm{C} 48-\mathrm{C} 49 & -178.3(3) & \mathrm{O} 2^{\prime}-\mathrm{C} 86^{\prime}-\mathrm{C} 87^{\prime}-\mathrm{C} 88^{\prime} & 51(3) \\ \mathrm{C} 52-\mathrm{C} 47-\mathrm{C} 48-\mathrm{C} 53 & -178.2(3) & \mathrm{C} 86^{\prime}-\mathrm{C} 87^{\prime}-\mathrm{C} 88^{\prime}-\mathrm{C} 89^{\prime} & -67(2) \\ \mathrm{N} 6-\mathrm{C} 47-\mathrm{C} 48-\mathrm{C} 53 & 2.3(4) & \mathrm{C} 86^{\prime}-\mathrm{O} 2^{\prime}-\mathrm{C} 89^{\prime}-\mathrm{C} 88^{\prime} & -33(3) \\ \mathrm{C} 47-\mathrm{C} 48-\mathrm{C} 49-\mathrm{C} 50 & -1.0(5) & \mathrm{C} 87^{\prime}-\mathrm{C} 88^{\prime}-\mathrm{C} 89^{\prime}-\mathrm{O} 2^{\prime} & 67(2) \\ & & & \end{array}$

Hydrogen-bond geometry $\left(\AA,{ }^{\circ}\right)$

\begin{tabular}{lllll}
\hline$D-\mathrm{H}^{\cdots} \cdots A$ & $D-\mathrm{H}$ & $\mathrm{H} \cdots A$ & $D \cdots A$ & $D-\mathrm{H}^{\cdots} A A$ \\
\hline $\mathrm{N} 8-\mathrm{H} 8 \cdots \mathrm{N} 9$ & 0.86 & 2.10 & $2.515(13)$ & 109 \\
$\mathrm{~N} 8{ }^{\prime}-\mathrm{H} 8{ }^{\prime} \cdots \mathrm{N} 9$ & 0.86 & 2.22 & $2.644(18)$ & 110 \\
\hline
\end{tabular}

(7-Methylindolin-1-ido- $\kappa N)\left(1,4,7,10,13,16\right.$-hexaoxacyclooctadecane- $\left.\kappa^{6} O\right)$ potassium (3)

Crystal data

$\left[\mathrm{K}\left(\mathrm{C}_{9} \mathrm{H}_{8} \mathrm{~N}\right)\left(\mathrm{C}_{12} \mathrm{H}_{24} \mathrm{O}_{6}\right)\right]$

$M_{r}=433.57$

Monoclinic, $P 2{ }_{1} / n$

$a=10.784$ (3) $\AA$

$b=9.754(3) \AA$

$c=21.783(7) \AA$

$\beta=91.864(4)^{\circ}$

$V=2290.1(12) \AA^{3}$

$Z=4$

\section{Data collection}

Bruker SMART CCD platform diffractometer

Radiation source: fine-focus sealed tube $\omega$ scans

Absorption correction: multi-scan

(SADABS; Krause et al., 2015)
$F(000)=928$

$D_{\mathrm{x}}=1.258 \mathrm{Mg} \mathrm{m}^{-3}$

Mo $K \alpha$ radiation, $\lambda=0.71073 \AA$

Cell parameters from 937 reflections

$\theta=2.8-25.0^{\circ}$

$\mu=0.27 \mathrm{~mm}^{-1}$

$T=173 \mathrm{~K}$

Block, red-brown

$0.24 \times 0.18 \times 0.15 \mathrm{~mm}$

$T_{\min }=0.843, T_{\max }=1.000$

21112 measured reflections

4071 independent reflections

2902 reflections with $I>2 \sigma(I)$

$R_{\text {int }}=0.062$

$\theta_{\max }=25.1^{\circ}, \theta_{\min }=1.9^{\circ}$ 
$h=-12 \rightarrow 12$

$k=-11 \rightarrow 11$

\section{Refinement}

Refinement on $F^{2}$

Least-squares matrix: full

$R\left[F^{2}>2 \sigma\left(F^{2}\right)\right]=0.059$

$w R\left(F^{2}\right)=0.170$

$S=1.05$

4071 reflections

355 parameters

195 restraints

Primary atom site location: structure-invariant direct methods $l=-25 \rightarrow 25$

Secondary atom site location: difference Fourier map

Hydrogen site location: inferred from neighbouring sites

$\mathrm{H}$-atom parameters constrained

$w=1 /\left[\sigma^{2}\left(F_{\mathrm{o}}^{2}\right)+(0.0838 P)^{2}+1.6493 P\right]$

where $P=\left(F_{\mathrm{o}}{ }^{2}+2 F_{\mathrm{c}}{ }^{2}\right) / 3$

$(\Delta / \sigma)_{\max }<0.001$

$\Delta \rho_{\max }=0.58$ e $\AA^{-3}$

$\Delta \rho_{\min }=-0.23$ e $\AA^{-3}$

Special details

Geometry. All esds (except the esd in the dihedral angle between two 1.s. planes) are estimated using the full covariance matrix. The cell esds are taken into account individually in the estimation of esds in distances, angles and torsion angles; correlations between esds in cell parameters are only used when they are defined by crystal symmetry. An approximate (isotropic) treatment of cell esds is used for estimating esds involving 1.s. planes.

Refinement. The 18-crown-6 macrocycle is also disordered (see below). The eight largest residual peaks are the two peaks near the $\mathrm{K}$ atom and those for six $\mathrm{O}$ atoms of the minor component of disorder. However, the data-to-parameter ratio drops below eight if this disorder is modeled. Thus only the anion disorder was modeled.

The anion is modeled as disordered with the planar flip of itself (0.905 (3):0.095 (3)). Analogous bond lengths and angles between the two positions of the disordered anion were restrained to be similar. Anisotropic displacement parameters for proximal atoms were restrained to be similar and restrained toward the expected motion relative to bond direction.

Fractional atomic coordinates and isotropic or equivalent isotropic displacement parameters $\left(\AA^{2}\right)$

\begin{tabular}{|c|c|c|c|c|c|}
\hline & $x$ & $y$ & $z$ & $U_{\text {iso }} * / U_{\text {eq }}$ & Occ. $(<1)$ \\
\hline K1 & $0.47867(6)$ & $0.10916(7)$ & $0.68763(3)$ & $0.0402(2)$ & \\
\hline N1 & $0.6799(3)$ & $0.1406(3)$ & $0.61289(13)$ & $0.0430(7)$ & $0.905(3)$ \\
\hline $\mathrm{C} 1$ & $0.7705(4)$ & $0.0421(5)$ & 0.61419 (19) & $0.0459(10)$ & $0.905(3)$ \\
\hline $\mathrm{H} 1$ & 0.765129 & -0.039544 & 0.637708 & $0.055^{*}$ & $0.905(3)$ \\
\hline $\mathrm{C} 2$ & $0.7249(8)$ & $0.2379(9)$ & $0.5741(6)$ & $0.0359(8)$ & $0.905(3)$ \\
\hline $\mathrm{C} 3$ & 0.6675 & $0.3618(4)$ & $0.55605(16)$ & $0.0432(9)$ & $0.905(3)$ \\
\hline $\mathrm{C} 4$ & $0.7309(4)$ & 0.4449 (4) & $0.51661(17)$ & $0.0515(9)$ & $0.905(3)$ \\
\hline $\mathrm{H} 4$ & 0.694709 & 0.529528 & 0.504037 & $0.062 *$ & $0.905(3)$ \\
\hline $\mathrm{C} 5$ & $0.8463(4)$ & $0.4090(4)$ & 0.49457 (19) & $0.0576(10)$ & $0.905(3)$ \\
\hline H5 & 0.886958 & 0.469650 & 0.467653 & $0.069 *$ & $0.905(3)$ \\
\hline C6 & $0.9020(4)$ & $0.2882(5)$ & $0.5110(2)$ & $0.0539(11)$ & $0.905(3)$ \\
\hline H6 & 0.980133 & 0.264237 & 0.495166 & $0.065^{*}$ & 0.905 (3) \\
\hline $\mathrm{C} 7$ & $0.8427(3)$ & $0.1996(4)$ & $0.55146(15)$ & $0.0427(8)$ & 0.905 (3) \\
\hline $\mathrm{C} 8$ & $0.5449(6)$ & $0.3985(6)$ & $0.5809(4)$ & $0.0589(13)$ & $0.905(3)$ \\
\hline H8A & 0.517177 & 0.486450 & 0.563550 & $0.088 *$ & $0.905(3)$ \\
\hline H8B & 0.552535 & 0.406093 & 0.625736 & $0.088 *$ & $0.905(3)$ \\
\hline $\mathrm{H} 8 \mathrm{C}$ & 0.484182 & 0.327207 & 0.569810 & $0.088 *$ & $0.905(3)$ \\
\hline $\mathrm{C} 9$ & $0.8698(3)$ & $0.0718(4)$ & $0.57852(17)$ & $0.0470(9)$ & $0.905(3)$ \\
\hline H9 & 0.941701 & 0.017349 & 0.573247 & $0.056^{*}$ & $0.905(3)$ \\
\hline $\mathrm{N} 1^{\prime}$ & 0.6079 (19) & $0.246(2)$ & $0.6119(10)$ & $0.034(5)$ & $0.095(3)$ \\
\hline $\mathrm{C} 1^{\prime}$ & $0.567(5)$ & $0.370(5)$ & 0.588 & $0.053(7)$ & $0.095(3)$ \\
\hline
\end{tabular}




\begin{tabular}{|c|c|c|c|c|c|}
\hline $\mathrm{H} 1^{\prime}$ & 0.491531 & 0.409632 & 0.599970 & $0.064 *$ & $0.095(3)$ \\
\hline $\mathrm{C} 2^{\prime}$ & $0.716(8)$ & $0.228(9)$ & $0.581(6)$ & $0.038(3)$ & $0.095(3)$ \\
\hline $\mathrm{C} 3^{\prime}$ & $0.800(2)$ & $0.119(3)$ & $0.5895(13)$ & $0.042(3)$ & $0.095(3)$ \\
\hline $\mathrm{C} 4^{\prime}$ & $0.904(3)$ & $0.121(3)$ & $0.5543(14)$ & $0.045(3)$ & $0.095(3)$ \\
\hline $\mathrm{H} 4^{\prime}$ & 0.961602 & 0.047874 & 0.557570 & $0.053 *$ & $0.095(3)$ \\
\hline $\mathrm{C} 5^{\prime}$ & $0.927(3)$ & $0.229(4)$ & $0.514(2)$ & $0.056(5)$ & $0.095(3)$ \\
\hline $\mathrm{H}^{\prime}$ & 0.999937 & 0.226918 & 0.490635 & $0.067^{*}$ & $0.095(3)$ \\
\hline $\mathrm{C6}^{\prime}$ & $0.849(3)$ & $0.337(4)$ & $0.5074(19)$ & $0.054(4)$ & $0.095(3)$ \\
\hline H6' & 0.865659 & 0.409039 & 0.479391 & $0.065^{*}$ & $0.095(3)$ \\
\hline$C 7^{\prime}$ & $0.741(2)$ & $0.342(3)$ & $0.5425(14)$ & $0.046(3)$ & $0.095(3)$ \\
\hline $\mathrm{C} 8^{\prime}$ & $0.777(4)$ & $0.011(4)$ & $0.6371(18)$ & $0.045(7)$ & $0.095(3)$ \\
\hline H8D & 0.849934 & -0.047214 & 0.642319 & $0.068 *$ & $0.095(3)$ \\
\hline H8E & 0.705141 & -0.044390 & 0.623772 & $0.068 *$ & $0.095(3)$ \\
\hline $\mathrm{H} 8 \mathrm{~F}$ & 0.759299 & 0.056058 & 0.676182 & $0.068 *$ & $0.095(3)$ \\
\hline $\mathrm{C} 9^{\prime}$ & $0.642(3)$ & $0.432(3)$ & $0.5462(15)$ & $0.047(3)$ & $0.095(3)$ \\
\hline $\mathrm{H} 9^{\prime}$ & 0.628112 & 0.516371 & 0.525073 & $0.056 *$ & $0.095(3)$ \\
\hline O1 & $0.4936(3)$ & $0.2527(4)$ & $0.80078(13)$ & $0.0813(9)$ & \\
\hline $\mathrm{C} 10$ & $0.5423(5)$ & $0.1834(6)$ & 0.85294 (19) & $0.0962(18)$ & \\
\hline $\mathrm{H} 10 \mathrm{~A}$ & 0.473722 & 0.140953 & 0.875365 & $0.115^{*}$ & \\
\hline H10B & 0.584995 & 0.249668 & 0.880795 & $0.115^{*}$ & \\
\hline $\mathrm{C} 11$ & $0.6316(5)$ & $0.0750(7)$ & $0.8346(2)$ & $0.0989(18)$ & \\
\hline H11A & 0.700394 & 0.117054 & 0.812171 & $0.119^{*}$ & \\
\hline H11B & 0.667180 & 0.028987 & 0.871642 & $0.119^{*}$ & \\
\hline $\mathrm{O} 2$ & 0.5698 & $-0.0206(3)$ & $0.79708(12)$ & $0.0761(8)$ & \\
\hline $\mathrm{C} 12$ & $0.6427(5)$ & $-0.1387(6)$ & $0.7868(2)$ & 0.1005 (19) & \\
\hline $\mathrm{H} 12 \mathrm{~A}$ & 0.663983 & -0.184421 & 0.826332 & $0.121^{*}$ & \\
\hline H12B & 0.720798 & -0.112539 & 0.767181 & $0.121^{*}$ & \\
\hline C13 & $0.5695(5)$ & $-0.2339(5)$ & $0.7458(2)$ & 0.0908 (16) & \\
\hline H13A & 0.616207 & -0.320085 & 0.740140 & $0.109 *$ & \\
\hline H13B & 0.489695 & -0.256513 & 0.764607 & $0.109 *$ & \\
\hline $\mathrm{O} 3$ & $0.5482(3)$ & -0.1709 & $0.69021(12)$ & $0.0670(7)$ & \\
\hline $\mathrm{C} 14$ & $0.4844(5)$ & $-0.2566(4)$ & $0.6477(3)$ & $0.0919(15)$ & \\
\hline H14A & 0.404047 & -0.284974 & 0.664362 & $0.110^{*}$ & \\
\hline H14B & 0.534049 & -0.340079 & 0.640399 & $0.110^{*}$ & \\
\hline $\mathrm{C} 15$ & $0.4630(5)$ & $-0.1815(5)$ & $0.5893(2)$ & $0.0866(14)$ & \\
\hline H15A & 0.541755 & -0.141718 & 0.575414 & $0.104 *$ & \\
\hline H15B & 0.430216 & -0.244508 & 0.557041 & $0.104 *$ & \\
\hline $\mathrm{O} 4$ & $0.3765(2)$ & -0.0765 & $0.59986(12)$ & $0.0652(7)$ & \\
\hline $\mathrm{C} 16$ & $0.3562(4)$ & $0.0063(4)$ & $0.54769(16)$ & $0.0627(10)$ & \\
\hline H16A & 0.336048 & -0.051851 & 0.511466 & $0.075^{*}$ & \\
\hline H16B & 0.432230 & 0.059113 & 0.539340 & $0.075 *$ & \\
\hline $\mathrm{C} 17$ & $0.2516(4)$ & $0.1019(5)$ & $0.55876(17)$ & $0.0686(11)$ & \\
\hline H17A & 0.232187 & 0.155687 & 0.521127 & $0.082 *$ & \\
\hline H17B & 0.176785 & 0.049111 & 0.569190 & $0.082 *$ & \\
\hline $\mathrm{O} 5$ & $0.2850(2)$ & 0.1903 & $0.60716(11)$ & $0.0604(7)$ & \\
\hline C18 & $0.1871(3)$ & $0.2743(4)$ & $0.62523(19)$ & $0.0658(10)$ & \\
\hline $\mathrm{H} 18 \mathrm{~A}$ & 0.123904 & 0.218585 & 0.645761 & $0.079 *$ & \\
\hline H18B & 0.147260 & 0.318732 & 0.588798 & $0.079 *$ & \\
\hline
\end{tabular}




\begin{tabular}{lllll} 
C19 & $0.2377(4)$ & $0.3800(4)$ & $0.6681(2)$ & $0.0797(13)$ \\
H19A & 0.300098 & 0.436117 & 0.647146 & $0.096^{*}$ \\
H19B & 0.170037 & 0.441474 & 0.680668 & $0.096^{*}$ \\
O6 & $0.2930(3)$ & $0.3180(3)$ & $0.72029(15)$ & $0.0772(8)$ \\
C20 & $0.3488(5)$ & $0.4134(5)$ & $0.7604(3)$ & $0.112(2)$ \\
H20A & 0.286899 & 0.482739 & 0.772129 & $0.134^{*}$ \\
H20B & 0.416627 & 0.461167 & 0.739537 & $0.134^{*}$ \\
C21 & $0.3987(6)$ & $0.3447(8)$ & $0.8154(3)$ & $0.131(3)$ \\
H21A & 0.431752 & 0.414059 & 0.844821 & $0.157^{*}$ \\
H21B & 0.331291 & 0.294250 & 0.835385 & $0.157^{*}$ \\
\hline
\end{tabular}

Atomic displacement parameters $\left(\AA^{2}\right)$

\begin{tabular}{|c|c|c|c|c|c|c|}
\hline & $U^{11}$ & $U^{22}$ & $U^{33}$ & $U^{12}$ & $U^{13}$ & $U^{23}$ \\
\hline K1 & $0.0465(4)$ & $0.0362(4)$ & $0.0378(4)$ & $-0.0007(3)$ & $0.0011(3)$ & $-0.0012(3)$ \\
\hline N1 & $0.0440(16)$ & $0.0398(16)$ & $0.0456(16)$ & $-0.0050(13)$ & $0.0062(13)$ & $-0.0020(12)$ \\
\hline $\mathrm{C} 1$ & $0.051(2)$ & $0.043(2)$ & $0.044(3)$ & $-0.0024(19)$ & -0.0018 (19) & $0.0023(18)$ \\
\hline $\mathrm{C} 2$ & $0.037(2)$ & $0.035(2)$ & $0.036(4)$ & $-0.006(2)$ & $-0.0028(14)$ & $-0.0074(15)$ \\
\hline $\mathrm{C} 3$ & $0.047(2)$ & $0.038(2)$ & $0.0442(19)$ & $-0.0057(17)$ & $-0.0059(16)$ & $-0.0084(16)$ \\
\hline $\mathrm{C} 4$ & $0.062(2)$ & $0.0396(19)$ & $0.052(2)$ & $-0.0041(17)$ & $-0.0126(18)$ & $0.0030(16)$ \\
\hline $\mathrm{C} 5$ & 0.059 (2) & $0.060(3)$ & $0.053(2)$ & -0.015 (2) & $-0.0036(18)$ & $0.015(2)$ \\
\hline C6 & $0.045(3)$ & $0.069(3)$ & $0.048(2)$ & $-0.009(2)$ & $0.003(2)$ & $0.010(2)$ \\
\hline $\mathrm{C} 7$ & $0.0396(18)$ & $0.049(2)$ & $0.0391(18)$ & $-0.0058(16)$ & $-0.0033(14)$ & $0.0011(15)$ \\
\hline $\mathrm{C} 8$ & $0.062(3)$ & $0.041(3)$ & $0.074(4)$ & $0.010(2)$ & $0.004(3)$ & $-0.005(2)$ \\
\hline C9 & $0.040(2)$ & $0.052(2)$ & $0.049(2)$ & $0.0037(16)$ & $-0.0017(16)$ & $0.0041(17)$ \\
\hline $\mathrm{N} 1^{\prime}$ & $0.037(10)$ & $0.025(9)$ & $0.039(12)$ & $-0.006(7)$ & $-0.002(8)$ & $-0.008(7)$ \\
\hline $\mathrm{C} 1^{\prime}$ & $0.059(13)$ & $0.032(13)$ & $0.069(15)$ & $0.003(9)$ & $0.004(12)$ & 0.005 (11) \\
\hline $\mathrm{C} 2^{\prime}$ & $0.038(6)$ & $0.036(6)$ & $0.040(7)$ & $-0.004(5)$ & $0.000(6)$ & $-0.005(6)$ \\
\hline $\mathrm{C} 3^{\prime}$ & $0.041(6)$ & $0.043(6)$ & $0.042(7)$ & $0.001(5)$ & $-0.004(6)$ & $-0.002(6)$ \\
\hline $\mathrm{C}^{\prime}$ & $0.040(6)$ & $0.050(7)$ & $0.043(7)$ & $-0.002(6)$ & $-0.004(6)$ & $0.001(6)$ \\
\hline $\mathrm{C} 5^{\prime}$ & $0.051(9)$ & $0.067(9)$ & $0.050(9)$ & $-0.004(7)$ & $-0.002(8)$ & $0.012(8)$ \\
\hline $\mathrm{C} 6^{\prime}$ & $0.051(6)$ & $0.061(7)$ & $0.049(7)$ & $-0.007(6)$ & $0.000(6)$ & $0.016(6)$ \\
\hline $\mathrm{C} 7^{\prime}$ & $0.051(6)$ & $0.042(6)$ & $0.044(7)$ & $-0.007(5)$ & $-0.005(5)$ & $-0.001(6)$ \\
\hline $\mathrm{C} 8^{\prime}$ & 0.039 (14) & $0.048(13)$ & 0.049 (16) & $0.021(11)$ & $0.012(12)$ & $0.004(10)$ \\
\hline $\mathrm{C} 9^{\prime}$ & $0.053(6)$ & $0.038(6)$ & $0.048(7)$ & $-0.005(6)$ & $-0.008(6)$ & $-0.001(6)$ \\
\hline $\mathrm{O} 1$ & $0.0748(19)$ & $0.103(2)$ & $0.0658(18)$ & $-0.0092(17)$ & $0.0074(14)$ & $-0.0332(16)$ \\
\hline $\mathrm{C} 10$ & $0.104(4)$ & $0.142(5)$ & $0.041(2)$ & $-0.052(4)$ & $-0.001(2)$ & $-0.022(3)$ \\
\hline $\mathrm{C} 11$ & $0.083(3)$ & $0.158(5)$ & $0.055(3)$ & $-0.017(4)$ & $-0.019(2)$ & $0.014(3)$ \\
\hline $\mathrm{O} 2$ & $0.0715(18)$ & $0.104(2)$ & $0.0522(15)$ & $0.0060(17)$ & $-0.0033(13)$ & $0.0143(15)$ \\
\hline $\mathrm{C} 12$ & $0.099(4)$ & $0.136(5)$ & $0.067(3)$ & $0.059(4)$ & $-0.001(3)$ & $0.038(3)$ \\
\hline $\mathrm{C} 13$ & $0.117(4)$ & $0.070(3)$ & $0.087(3)$ & $0.039(3)$ & $0.030(3)$ & $0.035(3)$ \\
\hline $\mathrm{O} 3$ & $0.0851(19)$ & $0.0446(14)$ & $0.0720(17)$ & $0.0067(13)$ & $0.0135(14)$ & $0.0112(13)$ \\
\hline $\mathrm{C} 14$ & $0.110(4)$ & $0.035(2)$ & $0.130(4)$ & $0.002(2)$ & $-0.002(3)$ & $-0.013(3)$ \\
\hline $\mathrm{C} 15$ & $0.111(4)$ & $0.056(3)$ & $0.092(3)$ & $0.006(3)$ & $-0.002(3)$ & $-0.032(2)$ \\
\hline $\mathrm{O} 4$ & $0.0704(17)$ & $0.0560(15)$ & $0.0691(17)$ & $-0.0098(13)$ & $0.0028(13)$ & $-0.0144(13)$ \\
\hline $\mathrm{C} 16$ & $0.064(2)$ & $0.080(3)$ & $0.0434(19)$ & $-0.008(2)$ & $-0.0008(16)$ & $-0.0116(19)$ \\
\hline $\mathrm{C} 17$ & $0.056(2)$ & $0.099(3)$ & $0.050(2)$ & $-0.016(2)$ & $-0.0069(17)$ & $0.002(2)$ \\
\hline O5 & $0.0475(14)$ & $0.0681(16)$ & $0.0655(16)$ & $0.0041(12)$ & $-0.0002(11)$ & $0.0055(13)$ \\
\hline
\end{tabular}


supporting information

\begin{tabular}{lllllll}
\hline C18 & $0.048(2)$ & $0.065(2)$ & $0.085(3)$ & $0.0133(18)$ & $0.0041(19)$ & $0.017(2)$ \\
C19 & $0.052(2)$ & $0.059(2)$ & $0.128(4)$ & $0.010(2)$ & $0.008(2)$ & $0.007(3)$ \\
O6 & $0.0682(18)$ & $0.0612(17)$ & $0.102(2)$ & $0.0043(14)$ & $0.0066(16)$ & $-0.0249(16)$ \\
C20 & $0.084(3)$ & $0.083(4)$ & $0.167(6)$ & $0.017(3)$ & $-0.018(4)$ & $-0.078(4)$ \\
C21 & $0.090(4)$ & $0.162(6)$ & $0.141(5)$ & $0.013(4)$ & $0.001(4)$ & $-0.104(5)$ \\
\hline
\end{tabular}

Geometric parameters $\left(\AA,{ }^{\circ}\right)$

\begin{tabular}{|c|c|c|c|}
\hline $\mathrm{K} 1-\mathrm{N} 1^{\prime}$ & $2.571(19)$ & $\mathrm{C} 8^{\prime}-\mathrm{H} 8 \mathrm{E}$ & 0.9800 \\
\hline $\mathrm{K} 1-\mathrm{N} 1$ & $2.772(3)$ & $\mathrm{C} 8^{\prime}-\mathrm{H} 8 \mathrm{~F}$ & 0.9800 \\
\hline $\mathrm{K} 1-\mathrm{O} 5$ & $2.797(2)$ & $\mathrm{C} 9^{\prime}-\mathrm{H} 9^{\prime}$ & 0.9500 \\
\hline $\mathrm{K} 1-\mathrm{O} 4$ & $2.831(3)$ & $\mathrm{O} 1-\mathrm{C} 21$ & $1.406(6)$ \\
\hline $\mathrm{K} 1-\mathrm{O} 3$ & $2.832(3)$ & $\mathrm{O} 1-\mathrm{C} 10$ & 1.409 (6) \\
\hline $\mathrm{K} 1-\mathrm{O} 1$ & $2.835(3)$ & $\mathrm{C} 10-\mathrm{C} 11$ & $1.493(8)$ \\
\hline $\mathrm{K} 1-\mathrm{O} 2$ & $2.846(3)$ & $\mathrm{C} 10-\mathrm{H} 10 \mathrm{~A}$ & 0.9900 \\
\hline $\mathrm{K} 1-\mathrm{O} 6$ & $2.959(3)$ & $\mathrm{C} 10-\mathrm{H} 10 \mathrm{~B}$ & 0.9900 \\
\hline $\mathrm{K} 1-\mathrm{C} 16$ & $3.432(4)$ & $\mathrm{C} 11-\mathrm{O} 2$ & $1.395(6)$ \\
\hline $\mathrm{K} 1-\mathrm{C}^{\prime}$ & $3.49(3)$ & $\mathrm{C} 11-\mathrm{H} 11 \mathrm{~A}$ & 0.9900 \\
\hline $\mathrm{N} 1-\mathrm{C} 1$ & $1.369(5)$ & $\mathrm{C} 11-\mathrm{H} 11 \mathrm{~B}$ & 0.9900 \\
\hline $\mathrm{N} 1-\mathrm{C} 2$ & $1.370(5)$ & $\mathrm{O} 2-\mathrm{C} 12$ & $1.418(6)$ \\
\hline $\mathrm{C} 1-\mathrm{C} 9$ & $1.374(6)$ & $\mathrm{C} 12-\mathrm{C} 13$ & $1.495(8)$ \\
\hline $\mathrm{C} 1-\mathrm{H} 1$ & 0.9500 & $\mathrm{C} 12-\mathrm{H} 12 \mathrm{~A}$ & 0.9900 \\
\hline $\mathrm{C} 2-\mathrm{C} 3$ & $1.408(6)$ & $\mathrm{C} 12-\mathrm{H} 12 \mathrm{~B}$ & 0.9900 \\
\hline $\mathrm{C} 2-\mathrm{C} 7$ & $1.427(5)$ & $\mathrm{C} 13-\mathrm{O} 3$ & $1.372(5)$ \\
\hline $\mathrm{C} 3-\mathrm{C} 4$ & $1.379(5)$ & $\mathrm{C} 13-\mathrm{H} 13 \mathrm{~A}$ & 0.9900 \\
\hline $\mathrm{C} 3-\mathrm{C} 8$ & $1.489(6)$ & C13-H13B & 0.9900 \\
\hline $\mathrm{C} 4-\mathrm{C} 5$ & $1.393(6)$ & $\mathrm{O} 3-\mathrm{C} 14$ & $1.410(5)$ \\
\hline $\mathrm{C} 4-\mathrm{H} 4$ & 0.9500 & $\mathrm{C} 14-\mathrm{C} 15$ & $1.480(7)$ \\
\hline $\mathrm{C} 5-\mathrm{C} 6$ & $1.365(6)$ & $\mathrm{C} 14-\mathrm{H} 14 \mathrm{~A}$ & 0.9900 \\
\hline $\mathrm{C} 5-\mathrm{H} 5$ & 0.9500 & $\mathrm{C} 14-\mathrm{H} 14 \mathrm{~B}$ & 0.9900 \\
\hline $\mathrm{C} 6-\mathrm{C} 7$ & $1.403(5)$ & $\mathrm{C} 15-\mathrm{O} 4$ & $1.408(5)$ \\
\hline C6- $\mathrm{H} 6$ & 0.9500 & $\mathrm{C} 15-\mathrm{H} 15 \mathrm{~A}$ & 0.9900 \\
\hline $\mathrm{C} 7-\mathrm{C} 9$ & $1.405(5)$ & $\mathrm{C} 15-\mathrm{H} 15 \mathrm{~B}$ & 0.9900 \\
\hline $\mathrm{C} 8-\mathrm{H} 8 \mathrm{~A}$ & 0.9800 & $\mathrm{O} 4-\mathrm{C} 16$ & $1.406(5)$ \\
\hline $\mathrm{C} 8-\mathrm{H} 8 \mathrm{~B}$ & 0.9800 & $\mathrm{C} 16-\mathrm{C} 17$ & $1.489(6)$ \\
\hline $\mathrm{C} 8-\mathrm{H} 8 \mathrm{C}$ & 0.9800 & $\mathrm{C} 16-\mathrm{H} 16 \mathrm{~A}$ & 0.9900 \\
\hline $\mathrm{C} 9-\mathrm{H} 9$ & 0.9500 & $\mathrm{C} 16-\mathrm{H} 16 \mathrm{~B}$ & 0.9900 \\
\hline $\mathrm{N} 1^{\prime}-\mathrm{C} 1^{\prime}$ & $1.374(19)$ & $\mathrm{C} 17-\mathrm{O} 5$ & $1.400(4)$ \\
\hline $\mathrm{N} 1^{\prime}-\mathrm{C} 2^{\prime}$ & $1.374(18)$ & $\mathrm{C} 17-\mathrm{H} 17 \mathrm{~A}$ & 0.9900 \\
\hline $\mathrm{C} 1^{\prime}-\mathrm{C} 9^{\prime}$ & $1.375(11)$ & $\mathrm{C} 17-\mathrm{H} 17 \mathrm{~B}$ & 0.9900 \\
\hline $\mathrm{C} 1^{\prime}-\mathrm{H} 1^{\prime}$ & 0.9500 & $\mathrm{O} 5-\mathrm{C} 18$ & $1.403(4)$ \\
\hline $\mathrm{C} 2^{\prime}-\mathrm{C} 3^{\prime}$ & 1.407 (19) & $\mathrm{C} 18-\mathrm{C} 19$ & $1.484(6)$ \\
\hline $\mathrm{C} 2^{\prime}-\mathrm{C} 7^{\prime}$ & $1.429(18)$ & $\mathrm{C} 18-\mathrm{H} 18 \mathrm{~A}$ & 0.9900 \\
\hline $\mathrm{C} 3^{\prime}-\mathrm{C} 4^{\prime}$ & $1.376(18)$ & $\mathrm{C} 18-\mathrm{H} 18 \mathrm{~B}$ & 0.9900 \\
\hline $\mathrm{C} 3^{\prime}-\mathrm{C} 8^{\prime}$ & $1.500(18)$ & $\mathrm{C} 19-\mathrm{O} 6$ & $1.403(5)$ \\
\hline $\mathrm{C} 4^{\prime}-\mathrm{C} 5^{\prime}$ & $1.398(19)$ & $\mathrm{C} 19-\mathrm{H} 19 \mathrm{~A}$ & 0.9900 \\
\hline $\mathrm{C} 4^{\prime}-\mathrm{H} 4^{\prime}$ & 0.9500 & С19-Н19B & 0.9900 \\
\hline $\mathrm{C} 5^{\prime}-\mathrm{C} 6^{\prime}$ & $1.358(19)$ & $\mathrm{O} 6-\mathrm{C} 20$ & $1.399(5)$ \\
\hline
\end{tabular}




\begin{tabular}{|c|c|c|c|}
\hline $\mathrm{C}^{\prime}-\mathrm{H} 5^{\prime}$ & 0.9500 & $\mathrm{C} 20-\mathrm{C} 21$ & $1.460(9)$ \\
\hline $\mathrm{C} 6^{\prime}-\mathrm{C} 7^{\prime}$ & $1.406(18)$ & $\mathrm{C} 20-\mathrm{H} 20 \mathrm{~A}$ & 0.9900 \\
\hline $\mathrm{C} 6^{\prime}-\mathrm{H} 6^{\prime}$ & 0.9500 & $\mathrm{C} 20-\mathrm{H} 20 \mathrm{~B}$ & 0.9900 \\
\hline $\mathrm{C} 7^{\prime}-\mathrm{C} 9^{\prime}$ & $1.396(18)$ & $\mathrm{C} 21-\mathrm{H} 21 \mathrm{~A}$ & 0.9900 \\
\hline $\mathrm{C} 8^{\prime}-\mathrm{H} 8 \mathrm{D}$ & 0.9800 & $\mathrm{C} 21-\mathrm{H} 21 \mathrm{~B}$ & 0.9900 \\
\hline $\mathrm{N} 1{ }^{\prime}-\mathrm{K} 1-\mathrm{O} 5$ & $81.9(5)$ & $\mathrm{C} 3^{\prime}-\mathrm{C} 8^{\prime}-\mathrm{H} 8 \mathrm{D}$ & 109.5 \\
\hline $\mathrm{N} 1-\mathrm{K} 1-\mathrm{O} 5$ & $100.57(8)$ & $\mathrm{C} 3^{\prime}-\mathrm{C} 8^{\prime}-\mathrm{H} 8 \mathrm{E}$ & 109.5 \\
\hline $\mathrm{N} 1{ }^{\prime}-\mathrm{K} 1-\mathrm{O} 4$ & $96.1(5)$ & $\mathrm{H} 8 \mathrm{D}-\mathrm{C} 8^{\prime}-\mathrm{H} 8 \mathrm{E}$ & 109.5 \\
\hline $\mathrm{N} 1-\mathrm{K} 1-\mathrm{O} 4$ & $88.22(8)$ & $\mathrm{C} 3^{\prime}-\mathrm{C} 8^{\prime}-\mathrm{H} 8 \mathrm{~F}$ & 109.5 \\
\hline $\mathrm{O} 5-\mathrm{K} 1-\mathrm{O} 4$ & $59.44(8)$ & $\mathrm{H} 8 \mathrm{D}-\mathrm{C} 88^{\prime}-\mathrm{H} 8 \mathrm{~F}$ & 109.5 \\
\hline $\mathrm{N} 11^{\prime}-\mathrm{K} 1-\mathrm{O} 3$ & $111.5(4)$ & $\mathrm{H} 8 \mathrm{E}-\mathrm{C} 8^{\prime}-\mathrm{H} 8 \mathrm{~F}$ & 109.5 \\
\hline $\mathrm{N} 1-\mathrm{K} 1-\mathrm{O} 3$ & $84.63(8)$ & $\mathrm{C} 1^{\prime}-\mathrm{C} 9^{\prime}-\mathrm{C} 7^{\prime}$ & $103.0(17)$ \\
\hline $\mathrm{O} 5-\mathrm{K} 1-\mathrm{O} 3$ & $118.50(8)$ & $\mathrm{C} 1^{\prime}-\mathrm{C} 9^{\prime}-\mathrm{H} 9^{\prime}$ & 128.5 \\
\hline $\mathrm{O} 4-\mathrm{K} 1-\mathrm{O} 3$ & $59.55(8)$ & $\mathrm{C} 7^{\prime}-\mathrm{C} 9^{\prime}-\mathrm{H} 9^{\prime}$ & 128.5 \\
\hline $\mathrm{N} 1{ }^{\prime}-\mathrm{K} 1-\mathrm{O} 1$ & $106.5(5)$ & $\mathrm{C} 21-\mathrm{O} 1-\mathrm{C} 10$ & $112.2(5)$ \\
\hline $\mathrm{N} 1-\mathrm{K} 1-\mathrm{O} 1$ & $115.63(9)$ & $\mathrm{C} 21-\mathrm{O} 1-\mathrm{K} 1$ & $119.4(3)$ \\
\hline $\mathrm{O} 5-\mathrm{K} 1-\mathrm{O} 1$ & $115.17(9)$ & $\mathrm{C} 10-\mathrm{O} 1-\mathrm{K} 1$ & $118.2(3)$ \\
\hline $\mathrm{O} 4-\mathrm{K} 1-\mathrm{O} 1$ & $155.96(9)$ & $\mathrm{O} 1-\mathrm{C} 10-\mathrm{C} 11$ & $110.5(4)$ \\
\hline $\mathrm{O} 3-\mathrm{K} 1-\mathrm{O} 1$ & $116.85(10)$ & $\mathrm{O} 1-\mathrm{C} 10-\mathrm{H} 10 \mathrm{~A}$ & 109.5 \\
\hline $\mathrm{N} 1^{\prime}-\mathrm{K} 1-\mathrm{O} 2$ & $126.2(5)$ & $\mathrm{C} 11-\mathrm{C} 10-\mathrm{H} 10 \mathrm{~A}$ & 109.5 \\
\hline $\mathrm{N} 1-\mathrm{K} 1-\mathrm{O} 2$ & $106.60(9)$ & $\mathrm{O} 1-\mathrm{C} 10-\mathrm{H} 10 \mathrm{~B}$ & 109.5 \\
\hline $\mathrm{O} 5-\mathrm{K} 1-\mathrm{O} 2$ & $151.81(8)$ & $\mathrm{C} 11-\mathrm{C} 10-\mathrm{H} 10 \mathrm{~B}$ & 109.5 \\
\hline $\mathrm{O} 4-\mathrm{K} 1-\mathrm{O} 2$ & $113.42(9)$ & $\mathrm{H} 10 \mathrm{~A}-\mathrm{C} 10-\mathrm{H} 10 \mathrm{~B}$ & 108.1 \\
\hline $\mathrm{O} 3-\mathrm{K} 1-\mathrm{O} 2$ & $58.04(9)$ & $\mathrm{O} 2-\mathrm{C} 11-\mathrm{C} 10$ & $109.2(4)$ \\
\hline $\mathrm{O} 1-\mathrm{K} 1-\mathrm{O} 2$ & $58.86(10)$ & $\mathrm{O} 2-\mathrm{C} 11-\mathrm{H} 11 \mathrm{~A}$ & 109.8 \\
\hline $\mathrm{N} 1{ }^{\prime}-\mathrm{K} 1-\mathrm{O} 6$ & $100.4(4)$ & $\mathrm{C} 10-\mathrm{C} 11-\mathrm{H} 11 \mathrm{~A}$ & 109.8 \\
\hline $\mathrm{N} 1-\mathrm{K} 1-\mathrm{O} 6$ & $127.88(9)$ & $\mathrm{O} 2-\mathrm{C} 11-\mathrm{H} 11 \mathrm{~B}$ & 109.8 \\
\hline $\mathrm{O} 5-\mathrm{K} 1-\mathrm{O} 6$ & $57.22(8)$ & $\mathrm{C} 10-\mathrm{C} 11-\mathrm{H} 11 \mathrm{~B}$ & 109.8 \\
\hline $\mathrm{O} 4-\mathrm{K} 1-\mathrm{O} 6$ & $110.57(8)$ & $\mathrm{H} 11 \mathrm{~A}-\mathrm{C} 11-\mathrm{H} 11 \mathrm{~B}$ & 108.3 \\
\hline $\mathrm{O} 3-\mathrm{K} 1-\mathrm{O} 6$ & $147.10(8)$ & $\mathrm{C} 11-\mathrm{O} 2-\mathrm{C} 12$ & $112.3(4)$ \\
\hline $\mathrm{O} 1-\mathrm{K} 1-\mathrm{O} 6$ & $58.04(9)$ & $\mathrm{C} 11-\mathrm{O} 2-\mathrm{K} 1$ & $109.8(3)$ \\
\hline $\mathrm{O} 2-\mathrm{K} 1-\mathrm{O} 6$ & $108.81(9)$ & $\mathrm{C} 12-\mathrm{O} 2-\mathrm{K} 1$ & $114.0(2)$ \\
\hline $\mathrm{N} 1{ }^{\prime}-\mathrm{K} 1-\mathrm{C} 16$ & $77.5(5)$ & $\mathrm{O} 2-\mathrm{C} 12-\mathrm{C} 13$ & $108.3(4)$ \\
\hline $\mathrm{N} 1-\mathrm{K} 1-\mathrm{C} 16$ & $78.26(9)$ & $\mathrm{O} 2-\mathrm{C} 12-\mathrm{H} 12 \mathrm{~A}$ & 110.0 \\
\hline $\mathrm{O} 5-\mathrm{K} 1-\mathrm{C} 16$ & $42.97(9)$ & $\mathrm{C} 13-\mathrm{C} 12-\mathrm{H} 12 \mathrm{~A}$ & 110.0 \\
\hline $\mathrm{O} 4-\mathrm{K} 1-\mathrm{C} 16$ & $23.51(9)$ & $\mathrm{O} 2-\mathrm{C} 12-\mathrm{H} 12 \mathrm{~B}$ & 110.0 \\
\hline $\mathrm{O} 3-\mathrm{K} 1-\mathrm{C} 16$ & $80.18(9)$ & $\mathrm{C} 13-\mathrm{C} 12-\mathrm{H} 12 \mathrm{~B}$ & 110.0 \\
\hline $\mathrm{O} 1-\mathrm{K} 1-\mathrm{C} 16$ & $157.82(10)$ & $\mathrm{H} 12 \mathrm{~A}-\mathrm{C} 12-\mathrm{H} 12 \mathrm{~B}$ & 108.4 \\
\hline $\mathrm{O} 2-\mathrm{K} 1-\mathrm{C} 16$ & $136.59(10)$ & $\mathrm{O} 3-\mathrm{C} 13-\mathrm{C} 12$ & $108.6(4)$ \\
\hline $\mathrm{O} 6-\mathrm{K} 1-\mathrm{C} 16$ & $99.85(9)$ & $\mathrm{O} 3-\mathrm{C} 13-\mathrm{H} 13 \mathrm{~A}$ & 110.0 \\
\hline $\mathrm{N} 1^{\prime}-\mathrm{K} 1-\mathrm{C} 1^{\prime}$ & $19.5(5)$ & $\mathrm{C} 12-\mathrm{C} 13-\mathrm{H} 13 \mathrm{~A}$ & 110.0 \\
\hline $\mathrm{O} 5-\mathrm{K} 1-\mathrm{C}^{\prime}$ & $67.6(12)$ & $\mathrm{O} 3-\mathrm{C} 13-\mathrm{H} 13 \mathrm{~B}$ & 110.0 \\
\hline $\mathrm{O} 4-\mathrm{K} 1-\mathrm{C} 1^{\prime}$ & $98.9(16)$ & $\mathrm{C} 12-\mathrm{C} 13-\mathrm{H} 13 \mathrm{~B}$ & 110.0 \\
\hline $\mathrm{O} 3-\mathrm{K} 1-\mathrm{C} 1^{\prime}$ & $129.5(8)$ & $\mathrm{H} 13 \mathrm{~A}-\mathrm{C} 13-\mathrm{H} 13 \mathrm{~B}$ & 108.3 \\
\hline $\mathrm{O} 1-\mathrm{K} 1-\mathrm{C} 1^{\prime}$ & $99.9(15)$ & $\mathrm{C} 13-\mathrm{O} 3-\mathrm{C} 14$ & $112.3(4)$ \\
\hline $\mathrm{O} 2-\mathrm{K} 1-\mathrm{C} 1^{\prime}$ & $138.5(14)$ & $\mathrm{C} 13-\mathrm{O} 3-\mathrm{K} 1$ & $119.1(3)$ \\
\hline $\mathrm{O} 6-\mathrm{K} 1-\mathrm{C} 1^{\prime}$ & $81.4(5)$ & $\mathrm{C} 14-\mathrm{O} 3-\mathrm{K} 1$ & $115.9(2)$ \\
\hline
\end{tabular}




\begin{tabular}{|c|c|c|c|}
\hline $\mathrm{C} 16-\mathrm{K} 1-\mathrm{C} 1^{\prime}$ & $76.5(16)$ & $\mathrm{O} 3-\mathrm{C} 14-\mathrm{C} 15$ & $109.3(4)$ \\
\hline $\mathrm{C} 1-\mathrm{N} 1-\mathrm{C} 2$ & $103.4(3)$ & $\mathrm{O} 3-\mathrm{C} 14-\mathrm{H} 14 \mathrm{~A}$ & 109.8 \\
\hline $\mathrm{C} 1-\mathrm{N} 1-\mathrm{K} 1$ & $118.8(2)$ & $\mathrm{C} 15-\mathrm{C} 14-\mathrm{H} 14 \mathrm{~A}$ & 109.8 \\
\hline $\mathrm{C} 2-\mathrm{N} 1-\mathrm{K} 1$ & $137.6(2)$ & $\mathrm{O} 3-\mathrm{C} 14-\mathrm{H} 14 \mathrm{~B}$ & 109.8 \\
\hline $\mathrm{N} 1-\mathrm{C} 1-\mathrm{C} 9$ & $114.1(4)$ & $\mathrm{C} 15-\mathrm{C} 14-\mathrm{H} 14 \mathrm{~B}$ & 109.8 \\
\hline $\mathrm{N} 1-\mathrm{C} 1-\mathrm{H} 1$ & 122.9 & $\mathrm{H} 14 \mathrm{~A}-\mathrm{C} 14-\mathrm{H} 14 \mathrm{~B}$ & 108.3 \\
\hline $\mathrm{C} 9-\mathrm{C} 1-\mathrm{H} 1$ & 122.9 & $\mathrm{O} 4-\mathrm{C} 15-\mathrm{C} 14$ & $107.8(4)$ \\
\hline $\mathrm{N} 1-\mathrm{C} 2-\mathrm{C} 3$ & $127.3(3)$ & $\mathrm{O} 4-\mathrm{C} 15-\mathrm{H} 15 \mathrm{~A}$ & 110.2 \\
\hline $\mathrm{N} 1-\mathrm{C} 2-\mathrm{C} 7$ & $111.6(3)$ & $\mathrm{C} 14-\mathrm{C} 15-\mathrm{H} 15 \mathrm{~A}$ & 110.2 \\
\hline $\mathrm{C} 3-\mathrm{C} 2-\mathrm{C} 7$ & $121.1(3)$ & $\mathrm{O} 4-\mathrm{C} 15-\mathrm{H} 15 \mathrm{~B}$ & 110.2 \\
\hline $\mathrm{C} 4-\mathrm{C} 3-\mathrm{C} 2$ & $117.1(4)$ & $\mathrm{C} 14-\mathrm{C} 15-\mathrm{H} 15 \mathrm{~B}$ & 110.2 \\
\hline $\mathrm{C} 4-\mathrm{C} 3-\mathrm{C} 8$ & $123.3(4)$ & $\mathrm{H} 15 \mathrm{~A}-\mathrm{C} 15-\mathrm{H} 15 \mathrm{~B}$ & 108.5 \\
\hline $\mathrm{C} 2-\mathrm{C} 3-\mathrm{C} 8$ & $119.5(4)$ & $\mathrm{C} 16-\mathrm{O} 4-\mathrm{C} 15$ & $111.8(3)$ \\
\hline $\mathrm{C} 3-\mathrm{C} 4-\mathrm{C} 5$ & $122.3(4)$ & $\mathrm{C} 16-\mathrm{O} 4-\mathrm{K} 1$ & $103.0(2)$ \\
\hline $\mathrm{C} 3-\mathrm{C} 4-\mathrm{H} 4$ & 118.9 & $\mathrm{C} 15-\mathrm{O} 4-\mathrm{K} 1$ & $109.3(2)$ \\
\hline $\mathrm{C} 5-\mathrm{C} 4-\mathrm{H} 4$ & 118.9 & $\mathrm{O} 4-\mathrm{C} 16-\mathrm{C} 17$ & $109.1(3)$ \\
\hline $\mathrm{C} 6-\mathrm{C} 5-\mathrm{C} 4$ & $121.1(4)$ & $\mathrm{O} 4-\mathrm{C} 16-\mathrm{K} 1$ & $53.46(16)$ \\
\hline $\mathrm{C} 6-\mathrm{C} 5-\mathrm{H} 5$ & 119.4 & $\mathrm{C} 17-\mathrm{C} 16-\mathrm{K} 1$ & $86.8(2)$ \\
\hline $\mathrm{C} 4-\mathrm{C} 5-\mathrm{H} 5$ & 119.4 & $\mathrm{O} 4-\mathrm{C} 16-\mathrm{H} 16 \mathrm{~A}$ & 109.9 \\
\hline $\mathrm{C} 5-\mathrm{C} 6-\mathrm{C} 7$ & $119.4(4)$ & $\mathrm{C} 17-\mathrm{C} 16-\mathrm{H} 16 \mathrm{~A}$ & 109.9 \\
\hline $\mathrm{C} 5-\mathrm{C} 6-\mathrm{H} 6$ & 120.3 & $\mathrm{~K} 1-\mathrm{C} 16-\mathrm{H} 16 \mathrm{~A}$ & 160.6 \\
\hline $\mathrm{C} 7-\mathrm{C} 6-\mathrm{H} 6$ & 120.3 & $\mathrm{O} 4-\mathrm{C} 16-\mathrm{H} 16 \mathrm{~B}$ & 109.9 \\
\hline $\mathrm{C} 6-\mathrm{C} 7-\mathrm{C} 9$ & $135.8(4)$ & $\mathrm{C} 17-\mathrm{C} 16-\mathrm{H} 16 \mathrm{~B}$ & 109.9 \\
\hline $\mathrm{C} 6-\mathrm{C} 7-\mathrm{C} 2$ & $119.0(4)$ & $\mathrm{K} 1-\mathrm{C} 16-\mathrm{H} 16 \mathrm{~B}$ & 73.4 \\
\hline $\mathrm{C} 9-\mathrm{C} 7-\mathrm{C} 2$ & $105.2(3)$ & $\mathrm{H} 16 \mathrm{~A}-\mathrm{C} 16-\mathrm{H} 16 \mathrm{~B}$ & 108.3 \\
\hline $\mathrm{C} 3-\mathrm{C} 8-\mathrm{H} 8 \mathrm{~A}$ & 109.5 & $\mathrm{O} 5-\mathrm{C} 17-\mathrm{C} 16$ & $109.3(3)$ \\
\hline $\mathrm{C} 3-\mathrm{C} 8-\mathrm{H} 8 \mathrm{~B}$ & 109.5 & $\mathrm{O} 5-\mathrm{C} 17-\mathrm{H} 17 \mathrm{~A}$ & 109.8 \\
\hline $\mathrm{H} 8 \mathrm{~A}-\mathrm{C} 8-\mathrm{H} 8 \mathrm{~B}$ & 109.5 & $\mathrm{C} 16-\mathrm{C} 17-\mathrm{H} 17 \mathrm{~A}$ & 109.8 \\
\hline $\mathrm{C} 3-\mathrm{C} 8-\mathrm{H} 8 \mathrm{C}$ & 109.5 & $\mathrm{O} 5-\mathrm{C} 17-\mathrm{H} 17 \mathrm{~B}$ & 109.8 \\
\hline $\mathrm{H} 8 \mathrm{~A}-\mathrm{C} 8-\mathrm{H} 8 \mathrm{C}$ & 109.5 & $\mathrm{C} 16-\mathrm{C} 17-\mathrm{H} 17 \mathrm{~B}$ & 109.8 \\
\hline $\mathrm{H} 8 \mathrm{~B}-\mathrm{C} 8-\mathrm{H} 8 \mathrm{C}$ & 109.5 & $\mathrm{H} 17 \mathrm{~A}-\mathrm{C} 17-\mathrm{H} 17 \mathrm{~B}$ & 108.3 \\
\hline $\mathrm{C} 1-\mathrm{C} 9-\mathrm{C} 7$ & $105.6(4)$ & $\mathrm{C} 17-\mathrm{O} 5-\mathrm{C} 18$ & $113.2(3)$ \\
\hline $\mathrm{C} 1-\mathrm{C} 9-\mathrm{H} 9$ & 127.2 & $\mathrm{C} 17-\mathrm{O} 5-\mathrm{K} 1$ & $117.8(2)$ \\
\hline $\mathrm{C} 7-\mathrm{C} 9-\mathrm{H} 9$ & 127.2 & $\mathrm{C} 18-\mathrm{O} 5-\mathrm{K} 1$ & $122.9(2)$ \\
\hline $\mathrm{C} 1^{\prime}-\mathrm{N} 1^{\prime}-\mathrm{C} 2^{\prime}$ & $101.3(16)$ & $\mathrm{O} 5-\mathrm{C} 18-\mathrm{C} 19$ & $108.5(3)$ \\
\hline $\mathrm{C} 1^{\prime}-\mathrm{N} 1^{\prime}-\mathrm{K} 1$ & $121.7(14)$ & $\mathrm{O} 5-\mathrm{C} 18-\mathrm{H} 18 \mathrm{~A}$ & 110.0 \\
\hline $\mathrm{C} 2^{\prime}-\mathrm{N} 1^{\prime}-\mathrm{K} 1$ & $136.8(15)$ & $\mathrm{C} 19-\mathrm{C} 18-\mathrm{H} 18 \mathrm{~A}$ & 110.0 \\
\hline $\mathrm{N} 1^{\prime}-\mathrm{C} 1^{\prime}-\mathrm{C} 9^{\prime}$ & $117.0(19)$ & $\mathrm{O} 5-\mathrm{C} 18-\mathrm{H} 18 \mathrm{~B}$ & 110.0 \\
\hline $\mathrm{N} 1{ }^{\prime}-\mathrm{C} 1^{\prime}-\mathrm{K} 1$ & $38.8(10)$ & $\mathrm{C} 19-\mathrm{C} 18-\mathrm{H} 18 \mathrm{~B}$ & 110.0 \\
\hline $\mathrm{C} 9^{\prime}-\mathrm{C} 1^{\prime}-\mathrm{K} 1$ & $155.7(16)$ & $\mathrm{H} 18 \mathrm{~A}-\mathrm{C} 18-\mathrm{H} 18 \mathrm{~B}$ & 108.4 \\
\hline $\mathrm{N} 1^{\prime}-\mathrm{C} 1^{\prime}-\mathrm{H} 1^{\prime}$ & 121.5 & $\mathrm{O} 6-\mathrm{C} 19-\mathrm{C} 18$ & $110.4(3)$ \\
\hline $\mathrm{C} 9^{\prime}-\mathrm{C} 1^{\prime}-\mathrm{H} 1^{\prime}$ & 121.5 & $\mathrm{O} 6-\mathrm{C} 19-\mathrm{H} 19 \mathrm{~A}$ & 109.6 \\
\hline $\mathrm{K} 1-\mathrm{C} 1^{\prime}-\mathrm{H} 1^{\prime}$ & 82.8 & $\mathrm{C} 18-\mathrm{C} 19-\mathrm{H} 19 \mathrm{~A}$ & 109.6 \\
\hline $\mathrm{N} 1^{\prime}-\mathrm{C} 2^{\prime}-\mathrm{C} 3^{\prime}$ & $126(2)$ & $\mathrm{O} 6-\mathrm{C} 19-\mathrm{H} 19 \mathrm{~B}$ & 109.6 \\
\hline $\mathrm{N} 1^{\prime}-\mathrm{C} 2^{\prime}-\mathrm{C} 7^{\prime}$ & $111.5(17)$ & $\mathrm{C} 18-\mathrm{C} 19-\mathrm{H} 19 \mathrm{~B}$ & 109.6 \\
\hline $\mathrm{C} 3^{\prime}-\mathrm{C} 2^{\prime}-\mathrm{C} 7^{\prime}$ & $121.9(19)$ & $\mathrm{H} 19 \mathrm{~A}-\mathrm{C} 19-\mathrm{H} 19 \mathrm{~B}$ & 108.1 \\
\hline $\mathrm{C} 4^{\prime}-\mathrm{C} 3^{\prime}-\mathrm{C} 2^{\prime}$ & $116.6(18)$ & $\mathrm{C} 20-\mathrm{O} 6-\mathrm{C} 19$ & $112.4(4)$ \\
\hline $\mathrm{C} 4^{\prime}-\mathrm{C} 3^{\prime}-\mathrm{C} 8^{\prime}$ & $124(2)$ & $\mathrm{C} 20-\mathrm{O} 6-\mathrm{K} 1$ & $109.1(3)$ \\
\hline
\end{tabular}




\begin{tabular}{|c|c|c|c|}
\hline $\mathrm{C} 2^{\prime}-\mathrm{C} 3^{\prime}-\mathrm{C} 8^{\prime}$ & $119(2)$ & $\mathrm{C} 19-\mathrm{O} 6-\mathrm{K} 1$ & $112.0(2)$ \\
\hline $\mathrm{C} 3^{\prime}-\mathrm{C} 4^{\prime}-\mathrm{C} 5^{\prime}$ & $122(2)$ & $\mathrm{O} 6-\mathrm{C} 20-\mathrm{C} 21$ & $110.3(5)$ \\
\hline $\mathrm{C} 3^{\prime}-\mathrm{C} 4^{\prime}-\mathrm{H} 4^{\prime}$ & 119.0 & $\mathrm{O} 6-\mathrm{C} 20-\mathrm{H} 20 \mathrm{~A}$ & 109.6 \\
\hline $\mathrm{C} 5^{\prime}-\mathrm{C} 4^{\prime}-\mathrm{H} 4^{\prime}$ & 119.0 & $\mathrm{C} 21-\mathrm{C} 20-\mathrm{H} 20 \mathrm{~A}$ & 109.6 \\
\hline $\mathrm{C} 6^{\prime}-\mathrm{C} 5^{\prime}-\mathrm{C} 4^{\prime}$ & $122(2)$ & $\mathrm{O} 6-\mathrm{C} 20-\mathrm{H} 20 \mathrm{~B}$ & 109.6 \\
\hline $\mathrm{C} 6^{\prime}-\mathrm{C} 5^{\prime}-\mathrm{H} 5^{\prime}$ & 119.1 & $\mathrm{C} 21-\mathrm{C} 20-\mathrm{H} 20 \mathrm{~B}$ & 109.6 \\
\hline $\mathrm{C} 4^{\prime}-\mathrm{C} 5^{\prime}-\mathrm{H} 5^{\prime}$ & 119.1 & $\mathrm{H} 20 \mathrm{~A}-\mathrm{C} 20-\mathrm{H} 20 \mathrm{~B}$ & 108.1 \\
\hline $\mathrm{C} 5^{\prime}-\mathrm{C} 6^{\prime}-\mathrm{C} 7^{\prime}$ & $119(2)$ & $\mathrm{O} 1-\mathrm{C} 21-\mathrm{C} 20$ & $111.0(5)$ \\
\hline $\mathrm{C} 5^{\prime}-\mathrm{C} 6^{\prime}-\mathrm{H} 6^{\prime}$ & 120.4 & $\mathrm{O} 1-\mathrm{C} 21-\mathrm{H} 21 \mathrm{~A}$ & 109.4 \\
\hline $\mathrm{C} 7^{\prime}-\mathrm{C} 6^{\prime}-\mathrm{H} 6^{\prime}$ & 120.4 & $\mathrm{C} 20-\mathrm{C} 21-\mathrm{H} 21 \mathrm{~A}$ & 109.4 \\
\hline $\mathrm{C} 9^{\prime}-\mathrm{C} 7^{\prime}-\mathrm{C} 6^{\prime}$ & $135(2)$ & $\mathrm{O} 1-\mathrm{C} 21-\mathrm{H} 21 \mathrm{~B}$ & 109.4 \\
\hline $\mathrm{C} 9^{\prime}-\mathrm{C} 7^{\prime}-\mathrm{C} 2^{\prime}$ & $107.1(16)$ & $\mathrm{C} 20-\mathrm{C} 21-\mathrm{H} 21 \mathrm{~B}$ & 109.4 \\
\hline $\mathrm{C} 6^{\prime}-\mathrm{C} 7^{\prime}-\mathrm{C} 2^{\prime}$ & $118.2(18)$ & $\mathrm{H} 21 \mathrm{~A}-\mathrm{C} 21-\mathrm{H} 21 \mathrm{~B}$ & 108.0 \\
\hline $\mathrm{C} 2-\mathrm{N} 1-\mathrm{C} 1-\mathrm{C} 9$ & $0.0(8)$ & $\mathrm{N} 1^{\prime}-\mathrm{C} 2^{\prime}-\mathrm{C} 7^{\prime}-\mathrm{C} 9^{\prime}$ & $-3(14)$ \\
\hline $\mathrm{K} 1-\mathrm{N} 1-\mathrm{C} 1-\mathrm{C} 9$ & $-176.0(3)$ & $\mathrm{C} 3^{\prime}-\mathrm{C} 2^{\prime}-\mathrm{C} 7^{\prime}-\mathrm{C} 9^{\prime}$ & $-178(11)$ \\
\hline $\mathrm{C} 1-\mathrm{N} 1-\mathrm{C} 2-\mathrm{C} 3$ & $179.4(12)$ & $\mathrm{N} 1^{\prime}-\mathrm{C} 2^{\prime}-\mathrm{C} 7^{\prime}-\mathrm{C} 6^{\prime}$ & $-179(8)$ \\
\hline $\mathrm{K} 1-\mathrm{N} 1-\mathrm{C} 2-\mathrm{C} 3$ & $-6(2)$ & $\mathrm{C} 3^{\prime}-\mathrm{C} 2^{\prime}-\mathrm{C} 7^{\prime}-\mathrm{C} 6^{\prime}$ & $6(18)$ \\
\hline $\mathrm{C} 1-\mathrm{N} 1-\mathrm{C} 2-\mathrm{C} 7$ & $0.1(12)$ & $\mathrm{N} 1^{\prime}-\mathrm{C} 1^{\prime}-\mathrm{C} 9^{\prime}-\mathrm{C} 7^{\prime}$ & $-1(8)$ \\
\hline $\mathrm{K} 1-\mathrm{N} 1-\mathrm{C} 2-\mathrm{C} 7$ & $174.9(3)$ & $\mathrm{K} 1-\mathrm{C} 1^{\prime}-\mathrm{C} 9^{\prime}-\mathrm{C} 7^{\prime}$ & $-4(15)$ \\
\hline $\mathrm{N} 1-\mathrm{C} 2-\mathrm{C} 3-\mathrm{C} 4$ & $179.6(10)$ & $\mathrm{C} 6^{\prime}-\mathrm{C} 7^{\prime}-\mathrm{C} 9^{\prime}-\mathrm{C} 1^{\prime}$ & $177(6)$ \\
\hline $\mathrm{C} 7-\mathrm{C} 2-\mathrm{C} 3-\mathrm{C} 4$ & $-1.1(16)$ & $\mathrm{C} 2^{\prime}-\mathrm{C} 7^{\prime}-\mathrm{C} 9^{\prime}-\mathrm{C} 1^{\prime}$ & $2(9)$ \\
\hline $\mathrm{N} 1-\mathrm{C} 2-\mathrm{C} 3-\mathrm{C} 8$ & $1.0(18)$ & $\mathrm{C} 21-\mathrm{O} 1-\mathrm{C} 10-\mathrm{C} 11$ & $173.2(5)$ \\
\hline $\mathrm{C} 7-\mathrm{C} 2-\mathrm{C} 3-\mathrm{C} 8$ & $-179.7(9)$ & $\mathrm{K} 1-\mathrm{O} 1-\mathrm{C} 10-\mathrm{C} 11$ & $27.9(5)$ \\
\hline $\mathrm{C} 2-\mathrm{C} 3-\mathrm{C} 4-\mathrm{C} 5$ & $0.7(9)$ & $\mathrm{O} 1-\mathrm{C} 10-\mathrm{C} 11-\mathrm{O} 2$ & $-61.3(5)$ \\
\hline $\mathrm{C} 8-\mathrm{C} 3-\mathrm{C} 4-\mathrm{C} 5$ & $179.2(5)$ & $\mathrm{C} 10-\mathrm{C} 11-\mathrm{O} 2-\mathrm{C} 12$ & $-169.5(4)$ \\
\hline $\mathrm{C} 3-\mathrm{C} 4-\mathrm{C} 5-\mathrm{C} 6$ & $0.4(6)$ & $\mathrm{C} 10-\mathrm{C} 11-\mathrm{O} 2-\mathrm{K} 1$ & $62.5(4)$ \\
\hline $\mathrm{C} 4-\mathrm{C} 5-\mathrm{C} 6-\mathrm{C} 7$ & $-0.9(6)$ & $\mathrm{C} 11-\mathrm{O} 2-\mathrm{C} 12-\mathrm{C} 13$ & $-179.4(4)$ \\
\hline $\mathrm{C} 5-\mathrm{C} 6-\mathrm{C} 7-\mathrm{C} 9$ & $-179.4(4)$ & $\mathrm{K} 1-\mathrm{O} 2-\mathrm{C} 12-\mathrm{C} 13$ & $-53.7(5)$ \\
\hline $\mathrm{C} 5-\mathrm{C} 6-\mathrm{C} 7-\mathrm{C} 2$ & $0.5(9)$ & $\mathrm{O} 2-\mathrm{C} 12-\mathrm{C} 13-\mathrm{O} 3$ & $63.4(5)$ \\
\hline $\mathrm{N} 1-\mathrm{C} 2-\mathrm{C} 7-\mathrm{C} 6$ & $180.0(7)$ & $\mathrm{C} 12-\mathrm{C} 13-\mathrm{O} 3-\mathrm{C} 14$ & $177.2(4)$ \\
\hline $\mathrm{C} 3-\mathrm{C} 2-\mathrm{C} 7-\mathrm{C} 6$ & $0.6(16)$ & $\mathrm{C} 12-\mathrm{C} 13-\mathrm{O} 3-\mathrm{K} 1$ & $-42.7(5)$ \\
\hline $\mathrm{N} 1-\mathrm{C} 2-\mathrm{C} 7-\mathrm{C} 9$ & $-0.2(12)$ & $\mathrm{C} 13-\mathrm{O} 3-\mathrm{C} 14-\mathrm{C} 15$ & $179.1(4)$ \\
\hline $\mathrm{C} 3-\mathrm{C} 2-\mathrm{C} 7-\mathrm{C} 9$ & $-179.6(10)$ & $\mathrm{K} 1-\mathrm{O} 3-\mathrm{C} 14-\mathrm{C} 15$ & $37.7(5)$ \\
\hline $\mathrm{N} 1-\mathrm{C} 1-\mathrm{C} 9-\mathrm{C} 7$ & $-0.1(5)$ & $\mathrm{O} 3-\mathrm{C} 14-\mathrm{C} 15-\mathrm{O} 4$ & $-68.6(5)$ \\
\hline $\mathrm{C} 6-\mathrm{C} 7-\mathrm{C} 9-\mathrm{C} 1$ & $180.0(4)$ & $\mathrm{C} 14-\mathrm{C} 15-\mathrm{O} 4-\mathrm{C} 16$ & $176.2(3)$ \\
\hline $\mathrm{C} 2-\mathrm{C} 7-\mathrm{C} 9-\mathrm{C} 1$ & $0.2(8)$ & $\mathrm{C} 14-\mathrm{C} 15-\mathrm{O} 4-\mathrm{K} 1$ & $62.8(4)$ \\
\hline $\mathrm{C} 2^{\prime}-\mathrm{N} 1^{\prime}-\mathrm{C} 1^{\prime}-\mathrm{C} 9^{\prime}$ & $-1(11)$ & $\mathrm{C} 15-\mathrm{O} 4-\mathrm{C} 16-\mathrm{C} 17$ & $172.0(3)$ \\
\hline $\mathrm{K} 1-\mathrm{N} 1^{\prime}-\mathrm{C} 1^{\prime}-\mathrm{C} 9^{\prime}$ & $-178(5)$ & $\mathrm{K} 1-\mathrm{O} 4-\mathrm{C} 16-\mathrm{C} 17$ & $-70.7(3)$ \\
\hline $\mathrm{C} 2^{\prime}-\mathrm{N} 1^{\prime}-\mathrm{C} 1^{\prime}-\mathrm{K} 1$ & $176(9)$ & $\mathrm{C} 15-\mathrm{O} 4-\mathrm{C} 16-\mathrm{K} 1$ & $-117.2(3)$ \\
\hline $\mathrm{C} 1^{\prime}-\mathrm{N} 1^{\prime}-\mathrm{C} 2^{\prime}-\mathrm{C} 3^{\prime}$ & $177(14)$ & $\mathrm{O} 4-\mathrm{C} 16-\mathrm{C} 17-\mathrm{O} 5$ & $63.9(4)$ \\
\hline $\mathrm{K} 1-\mathrm{N} 1^{\prime}-\mathrm{C} 2^{\prime}-\mathrm{C}^{\prime}$ & $-7(23)$ & $\mathrm{K} 1-\mathrm{C} 16-\mathrm{C} 17-\mathrm{O} 5$ & $14.5(3)$ \\
\hline $\mathrm{C} 1^{\prime}-\mathrm{N} 1^{\prime}-\mathrm{C} 2^{\prime}-\mathrm{C} 7^{\prime}$ & $3(13)$ & $\mathrm{C} 16-\mathrm{C} 17-\mathrm{O} 5-\mathrm{C} 18$ & $-173.5(3)$ \\
\hline $\mathrm{K} 1-\mathrm{N} 1^{\prime}-\mathrm{C} 2^{\prime}-\mathrm{C} 7^{\prime}$ & $178(3)$ & $\mathrm{C} 16-\mathrm{C} 17-\mathrm{O} 5-\mathrm{K} 1$ & $-20.2(4)$ \\
\hline $\mathrm{N} 1^{\prime}-\mathrm{C} 2^{\prime}-\mathrm{C} 3^{\prime}-\mathrm{C} 4^{\prime}$ & $-179(11)$ & $\mathrm{C} 17-\mathrm{O} 5-\mathrm{C} 18-\mathrm{C} 19$ & $-169.7(3)$ \\
\hline $\mathrm{C} 7^{\prime}-\mathrm{C} 2^{\prime}-\mathrm{C} 3^{\prime}-\mathrm{C} 4^{\prime}$ & $-5(17)$ & $\mathrm{K} 1-\mathrm{O} 5-\mathrm{C} 18-\mathrm{C} 19$ & $38.6(4)$ \\
\hline $\mathrm{N} 1^{\prime}-\mathrm{C} 2^{\prime}-\mathrm{C} 3^{\prime}-\mathrm{C} 8^{\prime}$ & $-2(19)$ & $\mathrm{O} 5-\mathrm{C} 18-\mathrm{C} 19-\mathrm{O} 6$ & $-60.4(4)$ \\
\hline $\mathrm{C} 7^{\prime}-\mathrm{C} 2^{\prime}-\mathrm{C} 3^{\prime}-\mathrm{C} 8^{\prime}$ & $172(10)$ & $\mathrm{C} 18-\mathrm{C} 19-\mathrm{O} 6-\mathrm{C} 20$ & $176.4(4)$ \\
\hline
\end{tabular}


supporting information

$\begin{array}{llll}\mathrm{C} 2^{\prime}-\mathrm{C} 3^{\prime}-\mathrm{C} 4^{\prime}-\mathrm{C} 5^{\prime} & 2(10) & \mathrm{C} 18-\mathrm{C} 19-\mathrm{O} 6-\mathrm{K} 1 & 53.1(4) \\ \mathrm{C} 8^{\prime}-\mathrm{C} 3^{\prime}-\mathrm{C} 4^{\prime}-\mathrm{C} 5^{\prime} & -175(4) & \mathrm{C} 19-\mathrm{O} 6-\mathrm{C} 20-\mathrm{C} 21 & 176.8(4) \\ \mathrm{C} 3^{\prime}-\mathrm{C} 4^{\prime}-\mathrm{C} 5^{\prime}-\mathrm{C} 6^{\prime} & 0(8) & \mathrm{K} 1-\mathrm{O} 6-\mathrm{C} 20-\mathrm{C} 21 & -58.3(5) \\ \mathrm{C} 4^{\prime}-\mathrm{C} 5^{\prime}-\mathrm{C} 6^{\prime}-\mathrm{C} 7^{\prime} & 1(9) & \mathrm{C} 10-\mathrm{O} 1-\mathrm{C} 21-\mathrm{C} 20 & -179.1(5) \\ \mathrm{C} 5^{\prime}-\mathrm{C} 6^{\prime}-\mathrm{C}^{\prime}-\mathrm{C} 9^{\prime} & -178(5) & \mathrm{K} 1-\mathrm{O} 1-\mathrm{C} 21-\mathrm{C} 20 & -34.3(7) \\ \mathrm{C} 5^{\prime}-\mathrm{C} 6^{\prime}-\mathrm{C} 7^{\prime}-\mathrm{C} 2^{\prime} & -4(11) & \mathrm{O} 6-\mathrm{C} 20-\mathrm{C} 21-\mathrm{O} 1 & 63.7(7)\end{array}$

